\title{
On the connection between two quasilinear elliptic problems with source terms of order 0 or 1
}

\author{
Haydar ABDEL HAMID* Marie Françoise BIDAUT-VERON ${ }^{\dagger}$
}

November 12, 2018

\begin{abstract}
We establish a precise connection between two elliptic quasilinear problems with Dirichlet data in a bounded domain of $\mathbb{R}^{N}$. The first one, of the form

$$
-\Delta_{p} u=\beta(u)|\nabla u|^{p}+\lambda f(x)+\alpha,
$$

involves a source gradient term with natural growth, where $\beta$ is nonnegative, $\lambda>0, f(x) \geqq 0$, and $\alpha$ is a nonnegative measure. The second one, of the form

$$
-\Delta_{p} v=\lambda f(x)(1+g(v))^{p-1}+\mu,
$$

presents a source term of order 0 , where $g$ is nondecreasing, and $\mu$ is a nonnegative measure. Here $\beta$ and $g$ can present an asymptote. The correlation gives new results of existence, nonexistence, regularity and multiplicity of the solutions for the two problems, without or with measures. New informations on the extremal solutions are given when $g$ is superlinear.
\end{abstract}

\section{Contents}

1 Introduction $\quad[3$

2 Notions of solutions $\quad 9$

2.1 Renormalized solutions . . . . . . . . . . . . . . . . . 9

2.2 Reachable solutions . . . . . . . . . . . . . . . . . . . . 11

2.3 Second member in $L^{1}(\Omega) \ldots \ldots \ldots \ldots \ldots \ldots \ldots \ldots \ldots \ldots$

2.4 More regularity results . . . . . . . . . . . . . . . . . 13

*Laboratoire de Mathématiques et Physique Théorique, CNRS UMR 6083, Faculté des Sciences, 37200 Tours France. E-mail address:veronmf@univ-tours.fr

${ }^{\dagger}$ Laboratoire de Mathématiques et Physique Théorique, CNRS UMR 6083, Faculté des Sciences, 37200 Tours France. E-mail address:abdelham@lmpt.univ-tours.fr 
3 Correlation between the two problems $\quad 15$

3.1 The pointwise change of unknowns . . . . . . . . . . . . . . . . 15

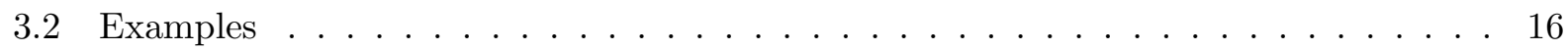

3.3 Proof of the correlation Theorem . . . . . . . . . . . . . . . 18

4 The case $\beta$ constant, g linear 2

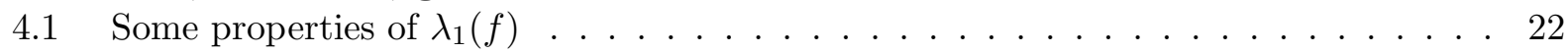

4.2 Proof of Theorem $1.2 \ldots \ldots \ldots \ldots \ldots \ldots \ldots \ldots$

5 Problem $(\mathrm{PV} \lambda)$ without measures $\quad 25$

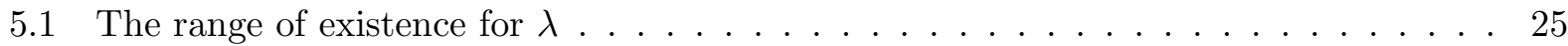

5.2 Cases where $g$ has a slow growth . . . . . . . . . . . . . . . 30

5.3 Superlinear case: Extremal solutions . . . . . . . . . . . . . . . . . 32

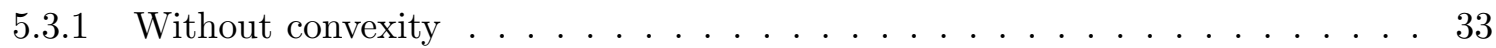

5.3 .2 With convexity . . . . . . . . . . . . . . . . 35

5.4 Boundedness and multiplicity under Sobolev conditions . . . . . . . . . . . . 40

6 Problem $(\mathrm{PV} \lambda)$ with measures 44

7 Applications to problem (PU $\lambda) \quad 47$

7.1 Remarks on growth assumptions . . . . . . . . . . . . . . . . 49

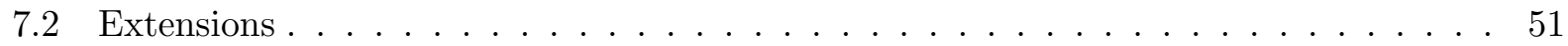

8 Appendix 


\section{Introduction}

Let $\Omega$ be a smooth bounded domain in $\mathbb{R}^{N}(N \geqq 2)$ and $1<p \leqq N$. In this paper we compare two quasilinear Dirichlet problems.

The first one presents a source gradient term with a natural growth:

$$
-\Delta_{p} u=\beta(u)|\nabla u|^{p}+\lambda f(x) \quad \text { in } \Omega, \quad u=0 \quad \text { on } \partial \Omega,
$$

where

$$
\beta \in C^{0}([0, L)), L \leqq \infty \text {, and } \beta \text { is nonnegative, } \beta \not \equiv 0 .
$$

and $\lambda>0$ is a given real, and

$$
f \in L^{1}(\Omega), \quad f \geqq 0 \text { a.e. in } \Omega .
$$

The function $\beta$ can have an asymptote at point $L$, and is not supposed to be increasing. For some results we suppose that $f$ belongs to suitable spaces $L^{r}(\Omega), r>1$.

The second problem involves a source term of order 0 , with the same $\lambda$ and $f$ :

$$
-\Delta_{p} v=\lambda f(x)(1+g(v))^{p-1} \quad \text { in } \Omega, \quad v=0 \quad \text { on } \partial \Omega .
$$

where

$$
g \in C^{1}([0, \Lambda)), \Lambda \leqq \infty, g(0)=0 \text { and } g \text { is nondecreasing, } g \not \equiv 0 .
$$

Here also $g$ can have an asymptote. In some cases where $\Lambda=\infty$, we make a growth condition on $g$ of the form

$$
M_{Q}=\lim \sup _{\tau \longrightarrow \infty} \frac{g(\tau)^{p-1}}{\tau^{Q}}<\infty
$$

for some $Q>0$, and setting $p^{*}=N p /(N-p)$, discuss according to the position of $Q$ with respect to $p-1$ and

$$
Q_{1}=\frac{N(p-1)}{N-p}, \quad Q^{*}=p^{*}-1=\frac{N(p-1)+p}{N-p}, \quad\left(Q_{1}=Q^{*}=\infty \text { if } p=N\right) .
$$

Problem (PU $\lambda)$ has been studied by many authors. Among them, let us mention the results of [13], 14] for the case $p=2$, [29], 30] for general quasilinear operators, when $\beta$ is defined on $\mathbb{R}$, not necessarily positive, but bounded. Problem (PU $\lambda$ ) has been studied in $[2]$ for $p=2$ and more general $\beta$, defined on $[0, \infty)$, such that $\underline{\lim }_{t \rightarrow \infty} \beta(t)>0$, see also many references therein. For general $p>1$, the problem has been investigated in [59] in the absorption case where $\beta(t) \leqq 0$ with measure data, and in [60] with a signed $\beta$, with strong growth assumptions on $|\beta|$.

Problem $(\mathrm{PV} \lambda)$ is also the object of a very rich litterature for $\Lambda=\infty$, especially when $g$ is superlinear, and convex, $p=2$, and $f \in L^{\infty}(\Omega)$. Here a main question is to give the range of $\lambda$ 
for which there exists at least one variational solution $v \in W_{0}^{1, p}(\Omega)$, or for which there exists a minimal bounded solution, and to get regularity properties of the limit of these solutions, called extremal solutions. For $p=2$, the case of the exponential $g(v)=e^{v}-1$ or of a power $g(v)=v^{q}$ has been studied first, see [20], [52], and the general case was investigated in [15], [16]. The regularity $L^{\infty}(\Omega)$ of the extremal solutions is also intensively discussed in many works, see [17] and references therein. Extensions to general p, are given in [33], 35], [28, [17] and [19, [18. A second question is the existence of a second solution when $g$ is subcritical with respect to the Sobolev exponent. It has been obtained for power-type nonlinearities of type concave-convex, see [6], [34], and [2] for general convex function $g$ and $p=2$, and some results are given in [28] for a power and $p>1$.

It is well known that a suitable change of variables problem (PU $\lambda$ ) leads formally to problem $(\operatorname{PV} \lambda)$, at least when $L=\infty$. Suppose for example that $\beta$ is a constant, that we can fix to $p-1$ :

$$
-\Delta_{p} u=(p-1)|\nabla u|^{p}+\lambda f(x) \quad \text { in } \Omega, \quad u=0 \quad \text { on } \partial \Omega .
$$

Setting $v=e^{u}-1$ leads formally to the problem

$$
-\Delta_{p} v=\lambda f(x)(1+v)^{p-1} \quad \text { in } \Omega, \quad v=0 \quad \text { on } \partial \Omega .
$$

and we can return from $v$ to $u$ by $u=\ln (1+v)$. However an example, due to [30], shows that the correspondence is more complex: assuming $f=0$ and $\Omega=B(0,1), p<N$, equation 1.4 admits the solution $u_{0} \equiv 0$, corresponding to $v_{0} \equiv 0$; but it has also an infinity of solutions:

$$
u_{m}(x)=\ln \left((1-m)^{-1}\left(|x|^{-(N-p) /(p-1)}-m\right)\right),
$$

defined for any $m \in(0,1)$, and $v_{m}=e^{u_{m}}-1$ satisfies

$$
-\Delta_{p} v_{m}=K_{m, N} \delta_{0} \quad \text { in } \mathcal{D}^{\prime}(\Omega),
$$

where $\delta_{0}$ is the Dirac mass concentrated at 0 , and $K_{m, N}>0$, thus $v_{m} \notin W_{0}^{1, p}(\Omega)$. Observe that $u_{m} \in W_{0}^{1, p}(\Omega)$ and it solves problem (1.4) in $\mathcal{D}^{\prime}(\Omega)$. Indeed the logarithmic singularity at 0 is not seen in $\mathcal{D}^{\prime}(\Omega)$.

In the case of a general $\beta$, the change of unknown in (PU $\lambda)$

$$
v(x)=\Psi(u(x))=\int_{0}^{u(x)} e^{\gamma(\theta) /(p-1)} d \theta, \quad \text { where } \gamma(t)=\int_{0}^{t} \beta(\theta) d \theta,
$$

leads formally to problem (PV $\lambda$ ), where $\Lambda=\Psi(L)$ and $g$ is given by

$$
g(v)=e^{\gamma\left(\Psi^{-1}(v)\right) /(p-1)}-1=\frac{1}{p-1} \int_{0}^{v} \beta(\Psi(s)) d s .
$$


It is apparently less used the converse correspondence, even in the case $p=2$ : for any function $g$ satisfying (1.2), the change of unknown

$$
u(x)=H(v(x))=\int_{0}^{v(x)} \frac{d s}{1+g(s)}
$$

leads formally to problem (PU $\lambda$ ), where $\beta$ satisfies (1.1) with $L=H(\Lambda)$; indeed $H=\Psi^{-1}$. And $\beta$ is linked to $g$ by relation (1.8), in other words

$$
\beta(u)=(p-1) g^{\prime}(v)=(p-1) g^{\prime}(\Psi(u)) .
$$

As a consequence, $\beta$ is nondecreasing if and only if $g$ is convex. Also the interval $[0, L)$ of definition of $\beta$ is finite if and only if $1 /(1+g) \in L^{1}(0, \Lambda)$. Some particular $\beta$ correspond to well known equations in $v$, where the main interesting ones are

$$
-\Delta_{p} v=\lambda f e^{v}, \quad-\Delta_{p} v=\lambda f(1+v)^{Q}, Q>p-1,
$$

where $\beta$ has an asymptote, or

$$
-\Delta_{p} v=\lambda f(1+v)^{Q}, Q<p-1, \quad-\Delta_{p} v=\lambda f(1+v)(1+\ln (1+v))^{p-1},
$$

where $\beta$ is defined on $[0, \infty)$.

Our aim is to precise the connection between problems ( $\mathrm{PU} \lambda)$ and problem (PV $\lambda)$, with possible measure data. As we see below, it allows to obtain new existence or nonexistence or multiplicity results, not only for problem (PU $\lambda$ ) but also for problem $(\mathrm{PV} \lambda)$.

In Section 2, we recall the notions of renormalized or reachable solutions, of problem

$$
-\Delta_{p} U=\mu \quad \text { in } \Omega, \quad U=0 \quad \text { on } \partial \Omega,
$$

when $\mu$ is a measure in $\Omega$. We give new regularity results when $\mu=F \in L^{m}(\Omega)$ for some $m>1$, see Lemma 2.13, or local estimates when $F \in L_{l o c}^{1}(\Omega)$, see Lemma 2.16, or when $F$ depends on $U$, see Proposition 2.14.

In Section 3 we prove the following correlation theorem between $u$ and $v$. We denote by $\mathcal{M}_{b}(\Omega)$ the set of bounded Radon measures, $\mathcal{M}_{s}(\Omega)$ the subset of measures concentrated on a set of $p$ capacity 0 , called singular; and $\mathcal{M}_{b}^{+}(\Omega)$ and $\mathcal{M}_{s}^{+}(\Omega)$ are the subsets on nonnegative ones.

Theorem 1.1 (i) Let $g$ be any function satisfying (1.2). Let $v$ be any renormalized solution of problem

$$
-\Delta_{p} v=\lambda f(x)(1+g(v))^{p-1}+\mu_{s} \quad \text { in } \Omega, \quad v=0 \quad \text { on } \partial \Omega,
$$


such that $0 \leqq v(x)<\Lambda$ a.e. in $\Omega$, where $\mu_{s} \in \mathcal{M}_{s}^{+}(\Omega)$. Then there exists $\alpha_{s} \in \mathcal{M}_{s}^{+}(\Omega)$, such that $u=H(v)$ is a renormalized solution of problem

$$
-\Delta_{p} u=\beta(u)|\nabla u|^{p}+\lambda f(x)+\alpha_{s} \quad \text { in } \Omega, \quad u=0 \quad \text { on } \partial \Omega,
$$

Moreover if $\mu_{s}=0$, then $\alpha_{s}=0$. If $\Lambda<\infty$, then $\mu_{s}=\alpha_{s}=0$ and $u, v \in W_{0}^{1, p}(\Omega) \cap L^{\infty}(\Omega)$. If $L<\infty=\Lambda$, then $\alpha_{s}=0$ and $u \in W_{0}^{1, p}(\Omega) \cap L^{\infty}(\Omega)$. If $L=\infty=\Lambda$ and $g$ is unbounded, then $\alpha_{s}=0$; if $g$ is bounded, then $\alpha_{s}=(1+g(\infty))^{1-p} \mu_{s}$.

(ii) Let $\beta$ be any function satisfying (1.1). Let $u$ be any renormalized solution of problem (1.12), such that $0 \leqq u(x)<L$ a.e. in $\Omega$, where $\alpha_{s} \in \mathcal{M}_{s}^{+}(\Omega)$. Then there exists $\mu \in \mathcal{M}^{+}(\Omega)$, such that $v=\Psi(u)$ is a reachable solution of problem

$$
-\Delta_{p} v=\lambda f(x)(1+g(v))^{p-1}+\mu \quad \text { in } \Omega, \quad v=0 \quad \text { on } \partial \Omega
$$

hence the equation holds in $\mathcal{D}^{\prime}(\Omega)$ ) and more precisely, for any $h \in W^{1, \infty}(\mathbb{R})$ such that $h^{\prime}$ has a compact support, and any $\varphi \in \mathcal{D}(\Omega)$,

$$
\int_{\Omega}|\nabla v|^{p-2} \nabla v \cdot \nabla(h(v) \varphi) d x=\int_{\Omega} h(v) \varphi \lambda f(x)(1+g(v))^{p-1} d x+h(\infty) \int_{\Omega} \varphi d \mu .
$$

Moreover if $L<\infty$, then $\alpha_{s}=0$ and $u \in W_{0}^{1, p}(\Omega) \cap L^{\infty}(\Omega)$. If $\Lambda<\infty$, then $\alpha_{s}=\mu=0$ and $u, v \in W_{0}^{1, p}(\Omega) \cap L^{\infty}(\Omega)$. If $L=\infty$ and $\beta \notin L^{1}((0, \infty))$, then $\alpha_{s}=0$; if $\beta \in L^{1}((0, \infty))$, then $\mu=e^{\gamma(\infty)} \alpha_{s}$ is singular, and $v$ is a renormalized solution. If $p=2$, or $p=N$, then in any case $\mu$ is singular.

This theorem precises and extends the results of [2, Theorems 4.2 and 4.3] where $p=2$ and $\beta$ is defined on $[0, \infty)$ and bounded from below near $\infty$. The proofs are different, based on the equations satisfied by the truncations of $u$ and $v$. The fact that $\alpha_{s}=0$ whenever $\beta \notin$ $L^{1}((0, \infty))$ also improves some results of [59]. In all the sequel we assume $f \not \equiv 0$.

In Section 4 we study the case $\beta$ constant, which means $g$ linear. The existence is linked to an eigenvalue problem with the weight $f$,

$$
-\Delta_{p} w=\lambda f(x)|w|^{p-2} w \quad \text { in } \Omega, \quad w=0 \quad \text { on } \partial \Omega,
$$

hence to the first eigenvalue

$$
\lambda_{1}(f)=\inf \left\{\left(\int_{\Omega}|\nabla w|^{p} d x\right) /\left(\int_{\Omega} f|w|^{p} d x\right): w \in W_{0}^{1, p}(\Omega) \backslash\{0\}\right\} .
$$

Theorem 1.2 Assume that $\beta(u) \equiv p-1$, or equivalently $g(v)=v$. 
(i) If $0<\lambda<\lambda_{1}(f)$ there exists a unique solution $v_{0} \in W_{0}^{1, p}(\Omega)$ to (1.5), and then a unique solution $u_{0} \in W_{0}^{1, p}(\Omega)$ to (1.4) such that $e^{u_{0}}-1 \in W_{0}^{1, p}(\Omega)$. If $f \in L^{N / p}(\Omega)$, then $u_{0}, v_{0} \in L^{k}(\Omega)$ for any $k>1$. If $f \in L^{r}(\Omega), r>N / p$, then $u_{0}$ and $v_{0} \in L^{\infty}(\Omega)$.

Moreover, if $f \in L^{r}(\Omega), r>N / p$, then for any measure $\mu_{s} \in \mathcal{M}_{s}^{+}(\Omega)$, there exists a renormalized solution $v_{s}$ of

$$
-\Delta_{p} v_{s}=\lambda f(x)\left(1+v_{s}\right)^{p-1}+\mu_{s} \quad \text { in } \Omega, \quad v_{s}=0 \quad \text { on } \partial \Omega
$$

thus there exists an infinity of solutions $u_{s}=\ln \left(1+v_{s}\right) \in W_{0}^{1, p}(\Omega)$ of (1.4), less regular than $u_{0}$.

(ii) If $\lambda>\lambda_{1}(f) \geqq 0$, or $\lambda=\lambda_{1}(f)>0$ and $f \in L^{N / p}(\Omega), p<N$, then (1.4), (1.5) and (1.17) admit no renormalized solution.

In Section 5 we study the existence of solutions of the problem (PV $\lambda$ ) for general $g$ without measures. It is easy to show that the set of $\lambda$ for which there exists a solution in $W_{0}^{1, p}(\Omega)$ is an interval $\left[0, \lambda^{*}\right)$ and the set of $\lambda$ for which there exists a minimal solution $\underline{v}_{\lambda} \in W_{0}^{1, p}(\Omega) \cap L^{\infty}(\Omega)$ such that $\left\|\underline{v}_{\lambda}\right\|_{L^{\infty}(\Omega)}<\Lambda$ is an interval $\left[0, \lambda_{b}\right)$.

The first important question is to know if $\lambda_{b}=\lambda^{*}$. One of the main results of this article is the extension of the well-known result of [15] relative to the case $p=2$, improving also a result of [19] for $p>1$.

Theorem 1.3 Assume that $g$ satisfies (1.2) and $g$ is convex near $\Lambda$, and $f \in L^{r}(\Omega), r>N / p$. There exists a real $\lambda^{*}>0$ such that

if $\lambda \in\left(0, \lambda^{*}\right)$ there exists a minimal bounded solution $\underline{v}_{\lambda}$ such $\left\|\underline{v}_{\lambda}\right\|_{L^{\infty}(\Omega)}<\Lambda$.

if $\lambda>\lambda^{*}$ there exists no renormalized solution. In particular it holds $\lambda_{b}=\lambda^{*}$.

Thus for $\lambda>\lambda^{*}$, not only there cannot exist variational solutions but also there cannot exist renormalized solutions, which is new for $p \neq 2$. It is noteworthy that the proof uses problem (PU $\lambda$ ) and is based on Theorem 1.1. A more general result is given at Theorem 5.8 .

When $\Lambda=\infty$ and $\lambda_{b}<\infty$, a second question is the regularity of the extremal function defined by $v^{*}=\lim _{\lambda} \lambda_{b} \underline{v}_{\lambda}$. Is it a solution of the limit problem, and in what sense? Is it variational, is it bounded? Under convexity assumptions we extend some results of [54], 64] and [2]:

Theorem 1.4 Assume that $g$ satisfies (1.2) with $\Lambda=\infty$ and $\lim _{t \rightarrow \infty} g(t) / t=\infty$, and $g$ is convex near $\infty$; and $f \in L^{r}(\Omega), r>N / p$. Then the extremal function $v^{*}=\lim _{\lambda} \lambda^{*} \underline{v}_{\lambda}$ is a renormalized solution of $\left(P V \lambda^{*}\right)$. Moreover

(i) If $N<p\left(1+p^{\prime}\right) /\left(1+p^{\prime} / r\right)$, then $v^{*} \in W_{0}^{1, p}(\Omega)$. If $N<p p^{\prime} /(1+1 /(p-1) r)$, then $v^{*}$ $\in W_{0}^{1, p}(\Omega) \cap L^{\infty}(\Omega)$.

(ii) If (1.3) holds with $Q<Q_{1}$, and $f \in L^{r}(\Omega)$ with $Q r^{\prime}<Q_{1}$, or if (1.3) holds with $Q<Q^{*}$, and $f \in L^{r}(\Omega)$ with $(Q+1) r^{\prime}<p^{*}$, then $v^{*} \in W_{0}^{1, p}(\Omega) \cap L^{\infty}(\Omega)$. 
The proof follows from Theorem 5.25. Propositions 5.27, 5.31 and 5.33. Without assumption of convexity on $g$, we obtain local results, see Theorem [5.17, based on regularity results of [10] and Harnack inequality.

When $\Lambda=\infty$ another question is the multiplicity of the variational solutions when $g$ is subcritical with respect to the Sobolev exponent. We prove the existence of at least two variational solutions in the following cases:

Theorem 1.5 Suppose that $g$ is defined on $[0, \infty)$, and $\lim _{t \rightarrow \infty} g(t) / t=\infty$, and that growth condition (1.3) holds with $Q<Q^{*}$, and $f \in L^{r}(\Omega)$ with $(Q+1) r^{\prime}<p^{*}$. Then

(i) if $g$ is convex near $\infty$, there exists $\lambda_{0}>0$ such that for any $\lambda<\lambda_{0}$, there exists at least two solutions $v \in W_{0}^{1, p}(\Omega) \cap L^{\infty}(\Omega)$ of $(P V \lambda)$.

(ii) If $p=2$ and $g$ is convex, or if $g$ satisfies the Ambrosetti-Rabinowitz condition (5.15) and $f \in L^{\infty}(\Omega)$, then for any $\lambda \in\left[0, \lambda^{*}\right)$ there exists at least two solutions $v \in W_{0}^{1, p}(\Omega) \cap L^{\infty}(\Omega)$ of $(P V \lambda)$.

This result is new even for $p=2$, improving results of [2] where the constraints on $g$ are stronger, and simplifying the proofs. In case $p>1$ and $g$ is of power-type, it solves the conjecture of [28] that $\lambda_{0}=\lambda^{*}$.

In Section 6 we study the existence for problem $(\mathrm{PV} \lambda)$ with measures, which requires a stronger growth assumption: (1.3) with $Q<Q_{1}$ :

Theorem 1.6 Suppose that $g$ is defined on $[0, \infty)$, and $f \in L^{r}(\Omega)$ with $r>N / p$. Let $\mu \in \mathcal{M}_{b}^{+}(\Omega)$ be arbitrary.

(i) Assume (1.3) with $Q=p-1$ and $M_{p-1} \lambda<\lambda_{1}(f)$, or with $Q<p-1$ and $Q r^{\prime}<Q_{1}$. Then problem

$$
-\Delta_{p} v=\lambda f(x)(1+g(v))^{p-1}+\mu \quad \text { in } \Omega, \quad v=0 \quad \text { on } \partial \Omega,
$$

admits a renormalized solution.

(ii) Assume (1.3) with $Q \in\left(p-1, Q_{1}\right)$ and $Q r^{\prime}<Q_{1}$. The same result is true if $\lambda$ and $|\mu|(\Omega)$ are small enough.

More generally we give existence results for problems where the unknown $U$ may be signed, of the form

$$
-\Delta_{p} U=\lambda h(x, U)+\mu \quad \text { in } \Omega, \quad U=0 \quad \text { on } \partial \Omega,
$$

where $\mu \in \mathcal{M}_{b}(\Omega)$, and $|h(x, U)| \leqq f(x)\left(1+|U|^{Q}\right)$, precising and improving the results announced in [39, see Theorem 6.2,

In Section 7, we return to problem (PU $\lambda$ ) for general $\beta$, and give existence, regularity, uniqueness or multiplicity results using Theorem 1.1 and the results of Sections 5 and 6 . 
We also analyse the meaning of the growth assumptions (1.3) for the function $g$ in terms of $\beta$. It was conjectured that if $\beta$ satisfying (1.1) with $L=\infty$, and is nondecreasing with $\lim _{t \rightarrow \infty} \beta(t)=\infty$, the function $g$ satisfies the growth condition (1.3) for any $Q>p-1$. We show that the conjecture is not true, and give sufficient conditions implying (1.3).

Finally we give some extensions where the function $f$ can also depend on $u$, or for problems with different powers of the gradient term.

\section{Notions of solutions}

\subsection{Renormalized solutions}

We refer to [25] for the main definitions, properties of regularity and existence of renormalized solutions. For any measure $\mu \in \mathcal{M}_{b}(\Omega)$ the positive part and the negative part of $\mu$ are denoted by $\mu^{+}$and $\mu^{-}$. The measure $\mu$ admits a unique decomposition

$$
\mu=\mu_{0}+\mu_{s}, \text { with } \mu_{0} \in \mathcal{M}_{0}(\Omega) \text { and } \mu_{s}=\mu_{s}^{+}-\mu_{s}^{-} \in \mathcal{M}_{s}(\Omega)
$$

where $\mathcal{M}_{0}(\Omega)$ is the subset of measures such that $\mu(B)=0$ for every Borel set $B \subseteq \Omega$ with $\operatorname{cap}_{p}(B, \Omega)=0$. If $\mu \geqq 0$, then $\mu_{0} \geqq 0$ and $\mu_{s} \geqq 0$. And any measure $\mu \in \mathcal{M}_{b}(\Omega)$ belongs to $\mathcal{M}_{0}(\Omega)$ if and only if it belongs to $L^{1}(\Omega)+W^{-1, p^{\prime}}(\Omega)$.

For any $k>0$ and $s \in \mathbb{R}$, we define the truncation

$$
T_{k}(s)=\max (-k, \min (k, s)) .
$$

If $U$ is measurable and finite a.e. in $\Omega$, and $T_{k}(U)$ belongs to $W_{0}^{1, p}(\Omega)$ for every $k>0$; we can define the gradient $\nabla U$ a.e. in $\Omega$ by

$$
\nabla T_{k}(U)=\nabla U \cdot \chi_{\{|U| \leqq k\}} \text { for any } k>0 .
$$

Then $U$ has a unique cap $_{p}$-quasi continuous representative; in the sequel $U$ will be identified to this representant. Next we recall two definitions of renormalized solutions among four equivalent ones given in [25]. The second one is mainly interesting, because it makes explicit the equation solved by the truncations $T_{k}(U)$ in the sense of distributions.

Definition 2.1 Let $\mu=\mu_{0}+\mu_{s}^{+}-\mu_{s}^{-} \in \mathcal{M}_{b}(\Omega)$. A function $U$ is a renormalized solution of problem

$$
-\Delta_{p} U=\mu \quad \text { in } \Omega, \quad U=0 \quad \text { on } \partial \Omega .
$$

if $U$ is measurable and finite a.e. in $\Omega$, such that $T_{k}(U)$ belongs to $W_{0}^{1, p}(\Omega)$ for any $k>0$, and $|\nabla U|^{p-1} \in L^{\tau}(\Omega)$, for any $\tau \in[1, N /(N-1))$, and one of the two (equivalent) conditions holds: 
(i) For any $h \in W^{1, \infty}(\mathbb{R})$ such that $h^{\prime}$ has a compact support, and any $\varphi \in W^{1, s}(\Omega)$ for some $s>N$, such that $h(U) \varphi \in W_{0}^{1, p}(\Omega)$,

$$
\int_{\Omega}|\nabla U|^{p-2} \nabla U \cdot \nabla(h(U) \varphi) d x=\int_{\Omega} h(U) \varphi d \mu_{0}+h(\infty) \int_{\Omega} \varphi d \mu_{s}^{+}-h(-\infty) \int_{\Omega} \varphi d \mu_{s}^{-} .
$$

(ii) For any $k>0$, there exist $\alpha_{k}, \beta_{k} \in \mathcal{M}_{0}(\Omega) \cap \mathcal{M}_{b}^{+}(\Omega)$, concentrated on the sets $\{U=k\}$ and $\{U=-k\}$ respectively, converging in the narrow topology to $\mu_{s}^{+}, \mu_{s}^{-}$such that for any $\psi \in$ $W_{0}^{1, p}(\Omega) \cap L^{\infty}(\Omega)$,

$$
\int_{\Omega}\left|\nabla T_{k}(U)\right|^{p-2} \nabla T_{k}(U) \cdot \nabla \psi d x=\int_{\{|U|<k\}} \psi d \mu_{0}+\int_{\Omega} \psi d \alpha_{k}-\int_{\Omega} \psi d \beta_{k} .
$$

that means, equivalently

$$
-\Delta_{p}\left(T_{k}(U)\right)=\mu_{0, k}+\alpha_{k}-\beta_{k} \quad \text { in } \mathcal{D}^{\prime}(\Omega)
$$

where $\mu_{0, k}=\mu_{0}\left\llcorner\{U \mid<k\}\right.$ is the restriction of $\mu_{0}$ to the set $\{|U|<k\}$.

Corresponding notions of local renormalized solutions are studied in [9]. The following properties are well-known in case $p<N$, see [7, 25] and more delicate in case $p=N$, see [36] and [45, where they require more regularity on the domain, namely, $\mathbb{R}^{N} \backslash \Omega$ is geometrically dense: $K_{N}(\Omega)=\inf \left\{r^{-N}|B(x, r) \backslash \Omega|: x \in \mathbb{R}^{N} \backslash \Omega, r>0\right\}>0$.

Proposition 2.2 Let $1<p \leqq N$, and $\mu \in \mathcal{M}_{b}(\Omega)$. Let $U$ be a renormalized solution of problem (2.2). If $p<N$, then for every $k>0$,

$$
\begin{aligned}
|\{|U| \geqq k\}| & \leqq C(N, p) k^{-(p-1) N /(N-p)}(|\mu|(\Omega))^{N /(N-p)} \\
|\{|\nabla U| \geqq k\}| & \leqq C(N, p) k^{-N(p-1) /(N-1)}(|\mu|(\Omega))^{N /(N-1)} .
\end{aligned}
$$

If $p=N$, then $U \in B M O$, and

$$
|\{|\nabla U| \geqq k\}| \leqq C\left(N, K_{N}(\Omega)\right) k^{-N}(|\mu|(\Omega))^{N /(N-1)} .
$$

Remark 2.3 As a consequence, if $p<N$, then for any $\sigma \in(0, N /(N-p)$ and $\tau \in(0, N /(N-1))$,

$$
\begin{aligned}
& \left(\int_{\Omega}|U|^{(p-1) \sigma} d x\right)^{1 / \sigma} \leqq C(N, p, \sigma)|\Omega|^{1 / \sigma-(N-p) / N}|\mu|(\Omega), \\
& \left(\int_{\Omega}|\nabla U|^{(p-1) \tau} d x\right)^{1 / \tau} \leqq C(N, p, \tau)|\Omega|^{1 / \tau-(N-1) / N}|\mu|(\Omega),
\end{aligned}
$$

If $p=N$, then $\sigma>0$ is arbitrary, and the constant also depends on $K_{\Omega}$. If $p>2-1 / N$, then $U \in W_{0}^{1, q}(\Omega)$ for every $q<(p-1) N /(N-1)$. 
Remark 2.4 Uniqueness of the solutions of (2.2) is still an open problem, when $p \neq 2, N$ and $\mu \notin \mathcal{M}_{0}(\Omega)$; see the recent results of [66], [49].

Otherwise, let $U \in W_{0}^{1, p}(\Omega)$, such that $-\Delta_{p} U=\mu$ in $\mathcal{D}^{\prime}(\Omega)$. Then $\mu \in W^{-1, p^{\prime}}(\Omega)$, hence $\mu \in \mathcal{M}_{0}(\Omega)$, and $U$ is an renormalized solution of (2.2).

Remark 2.5 Let $U$ be any renormalized solution of (2.2), where $\mu$ is given by (2.1).

(i) If $U \geqq 0$ a.e. in $\Omega$, then the singular part $\mu_{s} \geqq 0$, see [25, Definition 2.21]. This was also called Inverse Maximum Principle" in [57]. More generally, if $u \geqq A$ a.e. in $\Omega$ for some real $A$, there still holds $\mu_{s} \geqq 0$. Indeed $u-A$ is a local renormalized solution, and it follows from [9, Theorem 2.2].

(ii) If $U \in L^{\infty}(\Omega)$, then $U=T_{\|U\|_{L^{\infty}(\Omega)}}(U) \in W_{0}^{1, p}(\Omega)$, thus $\mu_{s}=0$ and $\mu=\mu_{0} \in \mathcal{M}_{0}(\Omega) \cap$ $W^{-1, p^{\prime}}(\Omega)$. As a consequence, if $L<\infty$, any solution $u$ of $(P U \lambda)$ is in $W_{0}^{1, p}(\Omega)$; if $\Lambda<\infty$, any solution of $(P V \lambda)$ is in $W_{0}^{1, p}(\Omega)$.

Many of our proofs are based on convergence results of [25]. Let us recall their main theorem:

Theorem 2.6 ([25]) Let $\mu=\mu_{0}+\mu_{s}^{+}-\mu_{s}^{-}$, with $\mu_{0}=F-\operatorname{div} g \in \mathcal{M}_{0}(\Omega), \quad \mu_{s}^{+}, \mu_{s}^{-} \in \mathcal{M}_{s}^{+}(\Omega)$. Let

$$
\mu_{n}=F_{n}-\operatorname{div} g_{n}+\rho_{n}-\eta_{n}, \quad \text { with } F_{n} \in L^{1}(\Omega), g_{n} \in\left(L^{p^{\prime}}(\Omega)\right)^{N}, \rho_{n}, \eta_{n} \in \mathcal{M}_{b}^{+}(\Omega) \text {. }
$$

Assume that $\left(F_{n}\right)$ converges to $F$ weakly in $L^{1}(\Omega),\left(g_{n}\right)$ converges to $g$ strongly in $\left(L^{p^{\prime}}(\Omega)\right)^{N}$ and $\left(\operatorname{div} g_{n}\right)$ is bounded in $\mathcal{M}_{b}(\Omega)$, and $\left(\rho_{n}\right)$ converges to $\mu_{s}^{+}$and $\left(\eta_{n}\right)$ converges to $\mu_{s}^{-}$in the narrow topology. Let $U_{n}$ be a renormalized solution of

$$
-\Delta_{p} U_{n}=\mu_{n} \quad \text { in } \Omega, \quad U_{n}=0 \quad \text { on } \partial \Omega .
$$

Then there exists a subsequence $\left(U_{\nu}\right)$ converging a.e. in $\Omega$ to a renormalized solution $U$ of problem

$$
-\Delta_{p} U=\mu \quad \text { in } \Omega, \quad U=0 \quad \text { on } \partial \Omega .
$$

And $\left(T_{k}\left(U_{\nu}\right)\right)$ converges to $T_{k}(U)$ strongly in $W_{0}^{1, p}(\Omega)$.

\subsection{Reachable solutions}

A weaker notion of solution will be used in the sequel, developped in [24, Theorems 1.1 and 1.2]:

Definition 2.7 Let $\mu \in \mathcal{M}_{b}(\Omega)$. A function $U$ is a reachable solution of problem (2.2) if it satisfies one of the (equivalent) conditions:

(i) There exists $\varphi_{n} \in \mathcal{D}(\Omega)$ and $U_{n} \in W_{0}^{1, p}(\Omega)$, such that $-\Delta_{p} U_{n}=\varphi_{n}$ in $W^{-1, p^{\prime}}(\Omega)$, such that $\left(\varphi_{n}\right)$ converges to $\mu$ weakly* in $\mathcal{M}_{b}(\Omega)$, and $\left(U_{n}\right)$ converges to $U$ a.e. in $\Omega$. 
(ii) $U$ is measurable and finite a.e. in $\Omega$, such that $T_{k}(U)$ belongs to $W_{0}^{1, p}(\Omega)$ for any $k>0$, and there exists $M>0$ such that $\int_{\Omega}\left|\nabla T_{k}(U)\right|^{p} d x \leqq M(k+1)$ for any $k>0$, and $|\nabla U|^{p-1} \in L^{1}(\Omega)$, and

$$
-\Delta_{p} U=\mu \quad \text { in } \mathcal{D}^{\prime}(\Omega) \text {. }
$$

(iii) $U$ is measurable and finite a.e., such that $T_{k}(U)$ belongs to $W_{0}^{1, p}(\Omega)$ for any $k>0$, and there exists $\mu_{0} \in \mathcal{M}_{0}(\Omega)$ and $\mu_{1}, \mu_{2} \in \mathcal{M}_{b}^{+}(\Omega)$, such that $\mu=\mu_{0}+\mu_{1}-\mu_{2}$ and for any $h \in W^{1, \infty}(\mathbb{R})$ such that $h^{\prime}$ has a compact support, and any $\varphi \in \mathcal{D}(\Omega)$,

$$
\int_{\Omega}|\nabla U|^{p-2} \nabla U \cdot \nabla(h(U) \varphi) d x=\int_{\Omega} h(U) \varphi d \mu_{0}+h(\infty) \int_{\Omega} \varphi d \mu_{1}-h(-\infty) \int_{\Omega} \varphi d \mu_{2} .
$$

Remark 2.8 Any reachable solution satisfies $|\nabla U|^{p-1} \in L^{\tau}(\Omega)$, for any $\tau \in[1, N /(N-1))$, and (the capp-quasi continuous representative of) $U$ is finite capp-quasi everywhere in $\Omega$, from 24 . Theorem 1.1] and [25, Remark 2.11]. Moreover, from [24], for any $k>0$, there exist $\alpha_{k}, \beta_{k} \in$ $\mathcal{M}_{0}(\Omega) \cap \mathcal{M}_{b}^{+}(\Omega)$, concentrated on the sets $\{U=k\}$ and $\{U=-k\}$ respectively, converging weakly* to $\mu_{1}, \mu_{2}$, such that

$$
-\Delta_{p}\left(T_{k}(U)\right)=\mu_{0, k}=\mu_{0}\left\llcorner\{|U|<k\}+\alpha_{k}-\beta_{k} \quad \text { in } \mathcal{D}^{\prime}(\Omega) .\right.
$$

Obviously, any renormalized solution is a reachable solution. The notions coincide for $p=2$ and $p=N$.

\subsection{Second member in $L^{1}(\Omega)$.}

In the sequel we often deal with the case where the second member is in $L^{1}(\Omega)$. Then the notion of renormalized solution coincides with the notions of reachable solution, and entropy solution introduced in [7], and SOLA solution given in [22], see also [12].

Definition 2.9 We call $\mathcal{W}(\Omega)$ the space of functions $U$ such that there exists $F \in L^{1}(\Omega)$ such that $U$ is a renormalized solution of problem

$$
-\Delta_{p} U=F \quad \text { in } \Omega, \quad U=0 \quad \text { on } \partial \Omega .
$$

Then $U$ is unique, we set

$$
U=\mathcal{G}(F)
$$

In the same way we call $\mathcal{W}_{\text {loc }}(\Omega)$ the space of fuctions $U$ such that there exists $F \in L_{\text {loc }}^{1}(\Omega)$ such that $U$ is a local renormalized solution of equation $-\Delta_{p} U=F$ in $\Omega$.

Remark 2.10 From uniqueness, the Comparison Principle holds:

If $U_{1}$ and $U_{2} \in \mathcal{W}(\Omega)$ and $-\Delta_{p} U_{1} \geqq-\Delta_{p} U_{2}$ a.e. in $\Omega$, then $U_{1} \geqq U_{2}$ a.e. in $\Omega$.

Remark 2.11 Theorem 2.6 implies in particular:

If $\left(F_{n}\right)$ converges to $F$ weakly in $L^{1}(\Omega)$, and $U_{n}=\mathcal{G}\left(F_{n}\right)$, then there exists a subsequence $\left(U_{\nu}\right)$ converging a.e. to some function $U$, such that $U=\mathcal{G}(F)$. 


\subsection{More regularity results}

All the proofs of this paragraph are given in the Appendix. First we deduce a weak form of the Picone inequality:

Lemma 2.12 Let $U \in W_{0}^{1, p}(\Omega)$, and $V \in \mathcal{W}(\Omega)$, such that $U \geqq 0$ and $-\Delta_{p} V \geqq 0$ a.e. in $\Omega$, and $V \not \equiv 0$. Then $U^{p}\left(-\Delta_{p} V\right) / V^{p-1} \in L^{1}(\Omega)$ and

$$
\int_{\Omega}|\nabla U|^{p} d x \geqq \int_{\Omega} U^{p} V^{1-p}\left(-\Delta_{p} V\right) d x
$$

Next we prove a regularity Lemma, giving estimates of $u$ and its gradient in optimal $L^{k}$ spaces, available for any renormalized solution. It improves the results of [11, [38, [4], [19] and extends the estimates of the gradient given in [46], [47] for solutions $U \in W_{0}^{1, p}(\Omega)$. Estimates in Marcinkiewicz or Lorentz spaces are given in [44, [5].

Lemma 2.13 Let $1<p \leqq N$. Let $U=\mathcal{G}(F)$ be the renormalized solution of problem

$$
-\Delta_{p} U=F \quad \text { in } \Omega, \quad U=0 \quad \text { on } \partial \Omega \text {. }
$$

with $F \in L^{m}(\Omega), 1<m<N$. Set $\bar{m}=N p /(N p-N+p)$.

(i) If $m>N / p$, then $U \in L^{\infty}(\Omega)$.

(ii) If $m=N / p$, then $U \in L^{k}(\Omega)$ for any $k \geqq 1$.

(iii) If $m<N / p$, then $U^{p-1} \in L^{k}(\Omega)$ for $k=N m /(N-p m)$.

(iv) $|\nabla U|^{(p-1)} \in L^{k}(\Omega)$ for $k=N m /(N-m)$. In particular if $\bar{m} \leqq m$, then $U \in W_{0}^{1, p}(\Omega)$.

Using this Lemma, we get regularity results under growth conditions, extending well known results in case $p=2, f \equiv 1$ :

Proposition 2.14 Let $1<p \leqq N$. Let $U=\mathcal{G}(h)$ where $h \in L^{1}(\Omega)$, and

$$
\mid h\left(x \mid \leqq f(x)\left(|U|^{Q}+1\right) \quad \text { a.e. in } \Omega,\right.
$$

with $f \in L^{r}(\Omega), r>1$ and $Q>0$. If $p<N$; then

(i) If $Q \geqq p-1$ and $Q r^{\prime}<Q_{1}$ (hence $\left.r>N / p\right)$, then $U \in W_{0}^{1, p}(\Omega) \cap L^{\infty}(\Omega)$.

(ii) If $Q>p-1$ and $Q r^{\prime}=Q_{1}$ and $|U|^{p-1} \in L^{\sigma}(\Omega)$ for some $\sigma>N /(N-p)$, then $U \in W_{0}^{1, p}(\Omega)$ and $U \in L^{k}(\Omega)$ for any $k \geqq 1$.

(iii) If $Q \geqq p-1$ and if $U \in W_{0}^{1, p}(\Omega)$, and $(Q+1) r^{\prime}<p^{*}$, then $U \in L^{\infty}(\Omega)$; if $(Q+1) r^{\prime}=p^{*}$, then $U \in L^{k}(\Omega)$ for any $k \geqq 1$.

(iv) If $Q<p-1$ and $r>N / p$, then $U \in W_{0}^{1, p}(\Omega) \cap L^{\infty}(\Omega)$. 
(v) If $Q<p-1$ and $r=N / p$, then $U \in W_{0}^{1, p}(\Omega)$ and $U \in L^{k}(\Omega)$ for any $k \geqq 1$.

(vi) If $Q<p-1$ and $r<N / p$ and $Q r^{\prime}<Q_{1}$, then $U^{k} \in L^{1}(\Omega)$ for any $k<d=N r(p-1-$ $Q) /(N-p r)$. Either $(Q+1) r^{\prime}<p^{*}$ and then $U \in W_{0}^{1, p}(\Omega)$, or $(Q+1) r^{\prime} \geqq p^{*}$, then $|\nabla U|^{t} \in L^{1}(\Omega)$ for any $t<\theta=N r(p-1-Q) /(N-(Q+1) r)$.

If $p=N$, then $U \in W_{0}^{1, N}(\Omega) \cap L^{\infty}(\Omega)$, and $|\nabla U|^{N(N-1) m /(N-m)} \in L^{1}(\Omega)$ for any $m<\min (r, N)$.

Remark 2.15 It may happen that $U \notin W_{0}^{1, p}(\Omega)$ for $Q \geqq p-1$, and condition (ii) is quite sharp: let $p=2$ and $\Omega=B(0,1)$; there exists a positive radial function $U \in L^{N /(N-2)}(\Omega)$ such that

$$
-\Delta U=U^{N /(N-2)} \quad \text { in } \Omega, \quad U=0 \quad \text { on } \partial \Omega, \quad \text { and }\left.\lim _{x \rightarrow 0}|x|^{N-2}|\ln | x\right|^{(N-2) / 2} U(x)=c_{N},
$$

where $c_{N}>0$, see [61]. Then $U \notin L^{\sigma}(\Omega)$ for $\sigma>N /(N-2)$, hence $U \notin W_{0}^{1,2}(\Omega)$. It satisfies the equation $-\Delta U=f U^{Q}$ with $Q=N /(N-2), f \equiv 1$, and also with $Q=1, f=U^{2 /(N-2)} \in L^{N / 2}(\Omega)$.

Next we we prove local estimates of the second member $F$ when $F \in L_{l o c}^{1}(\Omega)$ and $F \geqq 0$. following an idea of [10]:

Lemma 2.16 Let $U \in \mathcal{W}_{l o c}(\Omega)$ such that $-\Delta_{p} U=F \geqq 0$ a.e. in $\Omega$. For any $x_{0} \in \Omega$ and any ball $B\left(x_{0}, 4 \rho\right) \subset \Omega$, and any $\sigma \in(0, N /(N-p))$, there exists a constant $C=C(N, p, \sigma)$, such that

$$
\int_{B\left(x_{0}, \rho\right)} F d x \leq C \rho^{N(1-1 / \sigma)-p}\left(\int_{B\left(x_{0}, 2 \rho\right)} U^{(p-1) \sigma} d x\right)^{1 / \sigma} .
$$

If $U \in W_{\text {loc }}^{1, p}(\Omega)$, there exists a constant $C=C(N, p)$ such that

$$
\int_{B\left(x_{0}, \rho\right)} F d x \leqq C \rho^{N-p} \inf e s s_{B\left(x_{0}, \rho\right)} U^{p-1}
$$

Finally we mention a result of [58, which is a direct consequence of the Maximum Principle when $p=2$, but is not straightforward for $p \neq 2$, since no Comparison Principle is known for measures:

Lemma 2.17 Let $h$ be a Caratheodory function from $\Omega \times[0, \infty)$ into $[0, \infty)$. Let $\mu_{s} \in \mathcal{M}_{s}^{+}(\Omega)$ and $u$ be a renormalized nonnegative solution of

$$
-\Delta_{p} U=h(x, U)+\mu_{s} \quad \text { in } \Omega, \quad U=0 \quad \text { on } \partial \Omega .
$$

Suppose that $\sup _{t \in[0, u(x)]} h(x, t)=F(x) \in L^{1}(\Omega)$. Then there exists a renormalized nonnegative solution $V$ of

$$
-\Delta_{p} V=h(x, V) \quad \text { in } \Omega, \quad V=0 \quad \text { on } \partial \Omega .
$$




\section{Correlation between the two problems}

\subsection{The pointwise change of unknowns}

(i) Let $\beta$ satisfy (1.1). Let for any $t \in[0, L)$

$$
\Psi(t)=\int_{0}^{t} e^{\gamma(\theta) /(p-1)} d \theta, \quad \gamma(t)=\int_{0}^{t} \beta(\theta) d \theta ;
$$

then $\Psi([0, L))=[0, \Lambda), \Lambda=\Psi(L) \leqq \infty$, and the function

$$
\tau \in[0, \Lambda) \mapsto g(\tau)=e^{\gamma\left(\Psi^{-1}(\tau)\right) /(p-1)}-1=\frac{1}{p-1} \int_{0}^{\tau} \beta\left(\Psi^{-1}(s)\right) d s
$$

satisfies (5.10) and $\Psi^{-1}=H$, where

$$
H(\tau)=\int_{0}^{\tau} \frac{d s}{1+g(s)} .
$$

(ii) Conversely let $g$ satisfying (5.10), then $H([0, \Lambda))=[0, L), L=H(\Lambda)$, and the function $t \in$ $[0, L) \mapsto \beta(t)=(p-1) g^{\prime}\left(H^{-1}(t)\right)$ satisfies (1.1), and $H=\Psi^{-1}$ : indeed

$$
H(\tau)=\int_{0}^{\tau} \frac{d s}{1+g(s)}=\int_{0}^{\tau} e^{-\gamma\left(\Psi^{-1}(s)\right) /(p-1)} d s=\int_{0}^{\Psi^{-1}(\tau)} e^{-\gamma(\theta)) /(p-1)} \Psi^{\prime}(\theta) d \theta=\Psi^{-1}(\tau) .
$$

Then $\beta$ and $g$ are linked by the relations, at any point $\tau=\Psi(t)$,

$$
\beta(t)=(p-1) g^{\prime}(\tau), \quad 1+g(\tau)=e^{\gamma(t) /(p-1)} .
$$

In particular $\beta$ is nondecreasing if and only if $g$ is convex.

Remark 3.1 One easily gets the following properties:

$$
\begin{gathered}
L=\infty \Longrightarrow \Lambda=\infty ; \quad L<\infty \Longleftrightarrow 1 /(1+g(s)) \in L^{1}((0, \Lambda)) ; \\
\Lambda<\infty \Longleftrightarrow e^{\gamma(t) /(p-1)} \in L^{1}((0, L)) ; \quad \gamma(L)<\infty \Longleftrightarrow \beta \in L^{1}((0, L)) \Longleftrightarrow g \text { bounded; } \\
\underline{\lim }_{t \rightarrow L} \beta(t)>0 \Longrightarrow \underline{\lim }_{t \rightarrow \Lambda} g(s) / s>0 ; \\
\lim _{t \longrightarrow L} \beta(t)=\infty \Longrightarrow \lim _{s \longrightarrow \Lambda} g(s) / s=\infty, \quad \text { and conversely if } \beta \text { is nondecreasing near } L .
\end{gathered}
$$

If $\Lambda<\infty$, then

$$
\lim _{v \longrightarrow \Lambda} g(v)=\infty \quad \text { if } \beta(u) \notin L^{1}((0, L)) ; \quad \lim _{v \longrightarrow \Lambda} g(v)=e^{\gamma(L) /(p-1)}-1 \text { if } \beta(u) \in L^{1}((0, L)) .
$$

Notice that the correlation between $g$ and $\beta$ is not monotone; we only have: if $g_{1}^{\prime} \leqq g_{2}^{\prime}$, then $\beta_{1} \leqq \beta_{2}$. Also it is not symmetric between $u$ and $v$ : we always have $u \leqq v$; moreover $\nabla u=\nabla v /(1+g(v))$, thus $u$ can be expected more regular than $v$ when $\lim _{v \longrightarrow \Lambda} g(v)=\infty$. 
Remark 3.2 (i) If $u$ is a renormalized solution of (1.12), then by definition $\beta(u)|\nabla u|^{p} \in L^{1}(\Omega)$; if $v$ is a renormalized solution of (1.11), then $f(1+g(v))^{p-1} \in L^{1}(\Omega)$.

(ii)) For any $v \in W_{0}^{1, p}(\Omega)$, then $u=H(v) \in W_{0}^{1, p}(\Omega)$.

(iii) If $L=\infty$ and $\underline{\lim }_{t \rightarrow \infty} \beta(t)>0$, and $u$ is a renormalized solution of (1.12), then $u \in$ $W_{0}^{1, p}(\Omega) ;$ indeed $\beta(t) \geqq m>0$ for $t \geqq K_{0}>0$, thus

$$
\int_{\Omega}|\nabla u|^{p} d x=\int_{\left\{u \geqq K_{0}\right\}}|\nabla u|^{p} d x+\int_{\left\{u \leqq K_{0}\right\}}|\nabla u|^{p} d x \leqq \frac{1}{m} \int_{\Omega} \beta(u)|\nabla u|^{p} d x+\int_{\Omega}\left|\nabla T_{K_{0}}(u)\right|^{p} d x .
$$

\subsection{Examples}

Here we give examples, where the correlation can be given (quite) explicitely, giving good models for linking the behaviour of $\beta$ near $L$ and $g$ near $\Lambda$. The computation is easier by starting from a given function $g$ and computing $u$ from (1.9) and then $\beta$ by (1.10). The examples show how the correlation is sensitive with respect to $\beta$ : a small perturbation on $\beta$ can imply a very strong perturbation on $g$. Examples 1,2,5,6 are remarkable, since they lead to very well known equations in $v$. Example 10 is a model of a new type of problems in $v$, presenting a singularity, which can be qualified as quenching problem. The arrow $\leftrightarrow$ indicates the formal link between the two problems.

$\left.\mathbf{1}^{\circ}\right)$ Cases where $\beta$ is defined on $[0, \infty)(L=\infty=\Lambda)$.

1) $\beta$ constant, g linear:

Let $\beta(u)=p-1, g(v)=v, u=\ln (1+v)$,

$$
-\Delta_{p} u=(p-1)|\nabla u|^{p}+\lambda f(x) \quad \leftrightarrow \quad-\Delta_{p} v=\lambda f(x)(1+v)^{p-1} .
$$

2) $g$ of power type and sublinear:

Let $0<Q<p-1$; setting $\alpha=Q /(p-1)<1$, and $\beta(u)=(p-1) \alpha /(1+(1-\alpha) u)$, we find $(1+g(v))^{p-1}=(1+v)^{Q} ;$ and $(1-\alpha) u=(1+v)^{1-\alpha}-1:$

$$
-\Delta_{p} u=\frac{(p-1) \alpha}{1+(1-\alpha) u}|\nabla u|^{p}+\lambda f(x) \quad \leftrightarrow \quad-\Delta_{p} v=\lambda f(x)(1+v)^{Q} ;
$$

here $g$ is concave and unbounded, thus $\beta$ is nonincreasing, and $\beta(u) \sim C / u$ near $\infty$, thus $\beta \notin$ $L^{1}((0, \infty))$.

3) $\beta$ of power type, $g$ of logarithmic type.

Let $\beta(u)=(p-1) u^{m}, m>0$, then $\left.g(v) \sim C v(\ln v)\right)^{m /(m+1)}$, with $C=(m+1)^{m /(m+1)}$. Indeed integrating by parts $\int_{1}^{u} s^{-(m+1)} e^{s^{m+1} /(m+1)} d s$, we find that $v \sim u^{-m} e^{u^{m+1} /(m+1)}$ near $\infty$, then $\ln v \sim u^{m+1} /(m+1)$. 
Conversely let $1+g(v)=(1+C v)(1+\ln (1+C v))^{m /(m+1)}, m>0, C>0$, then $C u=(m+$ $1)\left((1+\ln (1+v))^{1 /(m+1)}-1\right)$, and $\beta(u)=(p-1) C\left((1+C u /(m+1))^{m}+m /(m+1+C u)\right)$, then $\beta(u) \sim K u^{m}$ near $\infty$, with $K=(p-1) C^{m+1}(m+1)^{-m}$.

4) $\beta$ of exponential type, $g$ of logarithmic type.

If $\beta(u)=(p-1) e^{u}$, then $g(v) \sim v \ln v$ near $\infty$. Indeed integrating by parts the integral $\int_{0}^{u} e^{-s} e^{e^{s}} d s$ we get $v \sim e^{e^{u}-u-1}$ near $\infty$.

If $\beta(u)=(p-1)\left(e^{u}+1\right)$, we find precisely $1+g(v)=(1+v)(1+\ln (1+v))$ and $u=\ln (1+\ln (1+v))$ :

$$
-\Delta_{p} u=\left(e^{u}+1\right)|\nabla u|^{p}+\lambda f(x) \quad \leftrightarrow \quad-\Delta_{p} v=\lambda f(x)((1+v)(1+\ln (1+v)))^{p-1} .
$$

If $\beta(u)=(p-1)\left(e^{e^{u}+u}+e^{u}+1\right)$, we verify that $e^{e+1} v=e^{e^{e^{u}}}-e^{e}$ and $\left.1+g(v) \sim v \ln v \ln (\ln v)\right)$ near $\infty$.

$\left.\mathbf{2}^{\circ}\right)$ Cases where $\beta$ has an asymptote $(L<\infty)$, but $g$ is defined on $[0, \infty)$. It is the case where $1 /(1+g(s)) \in L^{1}((0, \infty))$.

5) $g$ of power type and superlinear:

Let $Q>p-1$; setting $\alpha=Q /(p-1)>1$, and $\beta(u)=(p-1) \alpha /(1-(\alpha-1) u)$, with $L=1 /(\alpha-1)$, we find $(1+g(v))^{p-1}=(1+v)^{Q}$ and $(\alpha-1) u=1-(1+v)^{1-\alpha}$ :

$$
-\Delta_{p} u=\frac{(p-1) \alpha}{1-(\alpha-1) u}|\nabla u|^{p}+\lambda f(x) \quad \leftrightarrow \quad-\Delta_{p} v=\lambda f(x)(1+v)^{Q} .
$$

Another example is the case $\beta(u)=2(p-1) \operatorname{tg} u$. with $L=\pi / 2$, where $1+g(v)=1+v^{2}$, and $u=$ Arctgv .

6) $g$ of exponential type:

Let $\beta(u)=(p-1) /(1-u)$ with $L=1$, then $1+g(v)=e^{v}$, and $u=1-e^{-v}$ :

$$
-\Delta_{p} u=\frac{p-1}{1-u}|\nabla u|^{p}+\lambda f(x) \quad \leftrightarrow \quad-\Delta_{p} v=\lambda f(x) e^{v} .
$$

7) $g$ of logarithmic type:

Let $\beta(u)=(p-1) k /(1-u)^{k+1}, k>0$ with $L=1$, then we obtain $\left.g(v) \sim k v(\ln v)\right)^{(k+1) / k}$ near $\infty$. Conversely, if $1+g(v)=(1+k v)(1+\ln (1+k v))^{(k+1) / k}$, then $\beta(u)=(p-1)\left(k /(1-u)^{k+1}+\right.$ $(k+1) /(1-u))$, thus $\beta(u) \sim(p-1) k /(1-u)^{k+1}$ near 1 . Observe that $\beta$ has a stronger singularity than the one of example 6 , but $g$ has a slow growth.

8) $g$ of strong exponential type:

Let $\beta(u)=(1-u)^{-1}\left(1-(\ln (e /(1-u)))^{-1}\right)$ with $L=1$, then $1+g(v)=e^{e^{v}-v-1}, u=1-e^{1-e^{v}}$. Notice that $\beta$ has a singularity of the same type as the one example 6 . 
$\left.3^{\circ}\right)$ Cases where $\beta$ and $g$ have an asymptote $(L<\infty$ and $\Lambda<\infty)$.

$9)$ Let $Q>0$. Setting $\alpha=Q /(p-1)>0$, and $\beta(u)=(p-1) \alpha /(1-(\alpha+1) u)$, with $L=1 /(\alpha+1)$, we obtain $(1+g(v))^{p-1}=(1-v)^{-Q}$ and $(\alpha+1) u=1-(1-v)^{\alpha+1}$ :

$$
-\Delta_{p} u=\frac{(p-1) \alpha}{1-(\alpha+1) u}|\nabla u|^{p}+\lambda f(x) \quad \leftrightarrow \quad-\Delta_{p} v=\frac{\lambda f(x)}{(1-v)^{Q}}
$$

10) $\beta(u)=(p-1) u /\left(1-u^{2}\right)$, then $1+g(v)=1 / \cos v$, and $u=\sin v$.

\subsection{Proof of the correlation Theorem}

For proving Theorem 1.1, we cannot use approximations by regular functions, because of to the possible nonuniqueness of the solutions of (2.2) for $p \neq 2, N$, see Remark 2.4. Then we use the equations satisfied by the truncations. Such an argument was also used in [50] in order to simplify the proofs of [25].

Remark 3.3 (i) If $u$ is a solution of (1.12), where $0 \leqq u(x)<L$ a.e. in $\Omega$, and if $L<\infty$, then $\alpha_{s}=0$ from Remark [2.5, and $u=T_{L}(u) \in W_{0}^{1, p}(\Omega) \cap L^{\bar{\infty}}(\Omega)$. If $v$ is solution of (1.11) and $\Lambda<\infty$, then $\mu_{s}=0$ and $v=T_{\Lambda}(v) \in W_{0}^{1, p}(\Omega) \cap L^{\infty}(\Omega)$.

(ii) If $u$ is a solution of (1.12), the set $\{u=L\}$ has a p-capacity 0. It folllows from [25, Remark 2.11], if $L=\infty$, from [25, Proposition 2.1] applied to $(u-L)^{+}$if $L<\infty$. In the same way if $v$ is a solution of (1.11), the set $\{v=\Lambda\}$ has a p-capacity 0 .

Lemma 3.4 Suppose that $u$ is a renormalized solution of (1.12), where $0 \leqq u(x)<L$ a.e. in $\Omega$, or that $v=\Psi(u)$ is a renormalized solution of (1.11), where $0 \leqq v(x)<\Lambda$ a.e. in $\Omega$. For any $K>0, k>0$ there exists $\alpha_{K}, \mu_{k} \in \mathcal{M}_{0}(\Omega) \cap \mathcal{M}_{b}^{+}(\Omega)$ such that the truncations satisfy the equations

$$
\begin{gathered}
-\Delta_{p} T_{K}(u)=\beta\left(T_{K}(u)\right)\left|\nabla T_{K}(u)\right|^{p}+\lambda f \chi\{u<K\}+\alpha_{K} \quad \text { in } \mathcal{D}^{\prime}(\Omega), \\
-\Delta_{p} T_{k}(v)=\lambda f(1+g(v))^{p-1} \chi_{\{v<k\}}+\mu_{k} \quad \text { in } \mathcal{D}^{\prime}(\Omega),
\end{gathered}
$$

and

$$
\mu_{k}=(1+g(k))^{p-1} \alpha_{K}, \quad \text { for any } k=\Psi(K)>0 .
$$

Moreover, if $u$ is a solution of (1.12) then $\alpha_{K}$ converges in the narrow topology to $\alpha_{s}$ as $K \nearrow L$; if $v$ is a solution of (1.11) then $\mu_{k}$ converges in the narrow topology to $\mu_{s}$ as $k \nearrow \Lambda$.

Proof. (i) Let $v$ be a renormalized solution of (1.11), and $u=H(v)$. Then $f(1+g(v))^{p-1} \in$ $L^{1}(\Omega)$. For any $k \in(0, \Lambda)$, let $K=H(k)$, then $T_{k}(v) \in W_{0}^{1, p}(\Omega)$, and $T_{K}(u)=H\left(T_{k}(v)\right) \in W_{0}^{1, p}(\Omega)$. Observe that

$$
\left(1+g\left(T_{k}(v)\right)\right)^{p-1}=e^{\gamma\left(T_{K}(u)\right)}, \quad \text { and } \nabla T_{k}(v)=e^{\gamma\left(T_{K}(u)\right) /(p-1)} \nabla T_{K}(u) .
$$


Thus $\nabla v=e^{\gamma(u) /(p-1)} \nabla u$, then $|\nabla v|^{p-1}=e^{\gamma(u)}|\nabla u|^{p-1}$ a.e. in $\Omega$. From (2.4) (2.5), there exists $\mu_{k} \in \mathcal{M}_{0}(\Omega) \cap \mathcal{M}_{b}^{+}(\Omega)$, concentrated on $\{v=k\}$ such that $\mu_{k} \rightarrow \mu_{s}$ in the narrow topology as $k \longrightarrow \infty$, and $T_{k}(v)$ satisfies (3.5), that means

$$
\int_{\Omega}\left|\nabla T_{k}(v)\right|^{p-2} \nabla T_{k}(v) . \nabla \varphi d x=\lambda \int_{\{v<k\}} f(1+g(v))^{p-1} \varphi d x+\int_{\Omega} \varphi d \mu_{k},
$$

for any $\varphi \in W_{0}^{1, p}(\Omega) \cap L^{\infty}(\Omega)$. For given $\psi \in W_{0}^{1, p}(\Omega) \cap L^{\infty}(\Omega)$, taking $\varphi=e^{-\gamma\left(T_{K}(u)\right)} \psi$, we obtain

$$
\begin{aligned}
\int_{\Omega}\left|\nabla T_{K}(u)\right|^{p-2} \nabla T_{K}(u) . \nabla \psi d x & =\int_{\Omega} \beta\left(T_{K}(u)\right)\left|\nabla T_{K}(u)\right|^{p} \psi d x \\
& +\lambda \int_{\{U<k\}} f \psi d x+\frac{1}{(1+g(k))^{p-1}} \int_{\Omega} \psi d \mu_{k} .
\end{aligned}
$$

In other words, $T_{K}(u)$ satisfies equation (3.4) where $\alpha_{K}$ is given by (3.6). If $\Lambda<\infty$, then $\mu_{s}=0$, and $v=T_{\Lambda}(v) \in W_{0}^{1, p}(\Omega) \cap L^{\infty}(\Omega)$, and $\mu_{k}$ converges to 0 in $\mathcal{D}^{\prime}(\Omega)$ as $k \nearrow \Lambda$, hence weakly* in $\mathcal{M}_{b}(\Omega)$.And taking $\varphi=T_{k}(v)$,

$$
\begin{aligned}
\lim _{k \nearrow \Lambda} k \mu_{k}(\Omega) & =\lim _{k \nearrow \Lambda}\left(\int_{\Omega}\left|\nabla T_{k}(v)\right|^{p} d x-\int_{\Omega} \lambda f(1+g(v))^{p-1} v \chi_{\{v<k\}} d x\right) \\
& =\int_{\Omega}|\nabla v|^{p} d x-\int_{\Omega} \lambda f(1+g(v))^{p-1} v d x=0,
\end{aligned}
$$

thus $\mu_{k}$ converges to 0 in the narrow topology. Hence in any case ( $\Lambda$ finite or not), $\mu_{k}$ converges to $\mu_{s}$ in the narrow topology as $k \nearrow \Lambda$.

(ii) Let $u$ be a renormalized solution of (1.12) and $v=\Psi(u)$. Then $\beta(u)) \mid \nabla u)\left.\right|^{p} \in L^{1}(\Omega)$. For any $K \in(0, L)$, let $k=\Psi(K) \in(0, \Lambda)$. Then $T_{k}(v)=\Psi\left(T_{K}(u)\right) \in W_{0}^{1, p}(\Omega)$. From (2.4) (2.5), there exists $\alpha_{K} \in \mathcal{M}_{0}(\Omega) \cap \mathcal{M}_{b}^{+}(\Omega)$, concentrated on the set $\{u=K\}$, such that $\alpha_{K}$ converges to $\alpha_{s}$ in the narrow topology, as $k \longrightarrow \infty$, and $T_{K}(u)$ satisfies (3.4), that means

$$
\int_{\Omega}\left|\nabla T_{K}(u)\right|^{p-2} \nabla T_{K}(u) . \nabla \psi d x=\int_{\{U<K\}} \beta(u)|\nabla u|^{p} \psi d x+\int_{\{U<K\}} \lambda f \psi d x+\int_{\Omega} \psi d \alpha_{K},
$$

for any $\psi \in W_{0}^{1, p}(\Omega) \cap L^{\infty}(\Omega)$. Taking $\psi=e^{\gamma\left(T_{K}(U)\right)} \varphi$, with $\varphi \in W_{0}^{1, p}(\Omega) \cap L^{\infty}(\Omega)$,

$$
\begin{aligned}
\int_{\Omega}\left|\nabla T_{k}(v)\right|^{p-2} \nabla T_{k}(v) . \nabla \varphi d x & =\int_{\{U<K\}} \lambda f e^{\gamma\left(T_{K}(u)\right)} \varphi d x+\int_{\Omega} e^{\gamma\left(T_{K}(u)\right)} \varphi d \alpha_{K}, \\
& =\int_{\{v<k\}} \lambda f(1+g(v))^{p-1} \varphi d x+(1+g(k))^{p-1} \int_{\Omega} \varphi d \alpha_{K} \\
& =\int_{\{v<k\}} \lambda f(1+g(v))^{p-1} \varphi d x+\int_{\Omega} \varphi d \mu_{k},
\end{aligned}
$$


or equivalently (3.5) holds, where $\mu_{k}$ is given by (3.6). If $L<\infty$, then $\alpha_{s}=0$ from Remark 2.5, and $u=T_{L}(u) \in W_{0}^{1, p}(\Omega) \cap L^{\infty}(\Omega)$. And $T_{K}(u)$ converges to $u$ strongly in $W_{0}^{1, p}(\Omega)$ as $K \nearrow L$. Then $\Delta_{p} T_{K}(u)$ converges to $\Delta_{p} u$ in $W^{-1, p^{\prime}}(\Omega)$, and $\beta\left(T_{K}(u)\right)\left|\nabla T_{K}(u)\right|^{p}$ converges to $\left.\left.\beta(u)\right) \mid \nabla u\right)\left.\right|^{p}$ and $\lambda f \chi_{\{u<K\}}$ converges to $\lambda f$ strongly in $L^{1}(\Omega)$. Taking $\psi=T_{K}(u)$ in (3.8), it follows that

$$
\begin{aligned}
\lim _{K \nearrow L} K \alpha_{K}(\Omega) & =\lim _{K \nearrow L}\left(\int_{\Omega}\left|\nabla T_{K}(u)\right|^{p} d x-\int_{\Omega} \beta\left(T_{K}(u)\right)\left|\nabla T_{K}(u)\right|^{p} d x-\int_{\{u<K\}} \lambda f T_{K}(u) d x\right) \\
& =\int_{\Omega}|\nabla u|^{p} d x-\int_{\Omega} \beta(u)|\nabla u|^{p} d x-\int_{\Omega} \lambda f u d x=0,
\end{aligned}
$$

thus $\alpha_{K}$ converges to 0 in the narrow topology as $K \nearrow L$. Hence in any case ( $L$ finite or not), $\alpha_{K}$ converges to $\alpha_{s}$ in the narrow topology as $K \nearrow L$.

Proof of Theorem 1.1. (i) Let $v$ be a solution of (1.11), where $0 \leqq v(x)<\Lambda$ a.e. in $\Omega$, and $u=H(v)$. Taking $\varphi_{k}=1-1 /\left(1+g\left(T_{k}(s)\right)\right)^{p-1}$, as a test function in (3.5), we find

$$
\begin{aligned}
\int_{\{u<K\}} \beta(u)|\nabla u|^{p} d x & =(p-1) \int_{\{v<k\}} \frac{\left.g^{\prime}(v) \mid \nabla v\right)\left.\right|^{p}}{(1+g(v))^{p}} d x=\lambda \int_{\{v<k\}} f(1+g(v))^{p-1} \varphi_{k} d x+\phi(k) \int_{\Omega} d \mu_{k} \\
& \leqq \lambda \int_{\Omega} f(1+g(v))^{p-1} d x+\int_{\Omega} d \mu_{k} \leqq C
\end{aligned}
$$

where $C>0$ is independent of $k$; then $\beta(u)|\nabla u|^{p} \in L^{1}(\Omega)$. And from (3.6),$\alpha_{K}$ converges in the narrow topology to a singular measure $\alpha_{s}$ : either $\lim _{k \longrightarrow \infty} g(k)=\infty$, equivalently $L<\infty$ or $L=\infty, \beta \notin L^{1}((0, \infty))$, and then $\alpha_{s}=0$; or $g$ is bounded, equivalently $L=\infty$ and $\beta \in L^{1}((0, \infty))$ and then $\alpha_{s}=(1+g(\infty))^{p-1} \mu_{s}$.

Since $T_{k}(u) \in W_{0}^{1, p}(\Omega)$, it is also a renormalized solution of equation (3.4). From [25, Theorem $3.4]$ there exists a subsequence converging to a renormalized solution $U$ of

$$
-\Delta_{p} U=\beta(u)|\nabla u|^{p}+\lambda f+\alpha_{s}
$$

and $T_{k}(u)$ converges a.e in $\Omega$ to $u$, thus (the quasicontinuous representative of) $U$ is equal to $u$. Then $u$ is solution of (1.12).

(ii) Let $u$ be a solution of (1.12), where $0 \leqq u(x)<L$ a.e. in $\Omega$, and $v=\Psi(u)$. Taking $\psi=e^{\gamma\left(T_{K}(u)\right)}-1=\left(1+g\left(T_{k}(v)\right)^{p-1}-1\right.$ as a test function if (3.4), we get after simplification

$$
\begin{aligned}
& \int_{\Omega} \beta\left(T_{K}(u)\right)\left|\nabla T_{K}(u)\right|^{p} d x=\int_{\{u<K\}} \lambda f \psi d x+\int_{\Omega} \psi d \alpha_{K} \\
&=\int_{\{v<k\}} \lambda f\left((1+g(v))^{p-1}-1\right) d x+\left((1+g(k))^{p-1}-1\right) \int_{\Omega} d \alpha_{K} \\
& \quad=\int_{\{v<k\}} \lambda f\left((1+g(v))^{p-1}-1\right) d x+\mu_{k}(\Omega)-\alpha_{K}(\Omega) .
\end{aligned}
$$


Since $\beta(u)|\nabla u|^{p} \in L^{1}(\Omega)$, then $\phi=f(1+g(v))^{p-1} \in L^{1}(\Omega)$, and the measures $\mu_{k}$ are bounded independently of $k$. There exists a sequence $\left(k_{n}\right)$ converging to $\Lambda$ such that $\left(\mu_{k_{n}}\right)$ converges weakly* to a measure $\mu$. Let $v_{n}=T_{k_{n}}(v)$, then $\left(v_{n}\right)$ converges to $v=\Psi(u)$ a.e. in $\Omega$. From [25, Section 5.1] applied to $v_{n}=T_{k_{n}}(v)$, solution of (3.5) for $k=k_{n},\left|\nabla v_{n}\right|^{p-1}$ is bounded in $L^{\tau}(\Omega)$ for any $\tau<N /(N-1)$, and $\nabla v_{n}$ converges to $\nabla v$ a.e. in $\Omega$, and $\left|\nabla v_{n}\right|^{p-2} \nabla v_{n}$ converges to $|\nabla v|^{p-2} \nabla v$ strongly in $L^{\tau}(\Omega)$ for any $\tau<N /(N-1)$. And $\lambda f\left((1+g(v))^{p-1} \chi_{\left\{v<k_{n}\right\}}\right.$ converges to $\phi$ strongly in $L^{1}(\Omega)$ from the Lebesgue Theorem. Then $v$ satisfies

$$
-\Delta_{p} v=\phi+\mu \quad \text { in } \mathcal{D}^{\prime}(\Omega) ;
$$

thus $\mu$ is uniquely determined, and $\mu_{k}$ converges weakly* to $\mu$ as $k \nearrow \Lambda$. Then $v$ is reachable solution of this equation. Let us set $M=\phi+\mu$.

Case $p=2$ or $p=N$. Then from uniqueness, $v$ is a renormalized solution of (3.10). There exists $m \in \mathcal{M}_{0}^{+}(\Omega)$ et $\eta_{s} \in \mathcal{M}_{s}^{+}(\Omega)$ such that $M=m+\eta_{s}$, and from the definition of renormalized solution, for any $k>0$, there exists $\eta_{k} \in \mathcal{M}_{0}^{+}(\Omega)$ concentrated on the set $\{v=k\}$, converging to $\eta_{s}$ in the narrow topology, and

$$
-\Delta_{p} T_{k}(v)=m\left\llcorner\left\{v<k_{n}\right\}+\eta_{k_{n}} \quad \text { in } \mathcal{D}^{\prime}(\Omega),\right.
$$

but we have also

$$
-\Delta_{p} T_{k_{n}}(v)=\lambda f(1+g(v))^{p-1} \chi_{\left\{v<k_{n}\right\}}+\mu_{k_{n}} \quad \text { in } \mathcal{D}^{\prime}(\Omega),
$$

thus $\eta_{k_{n}}=\mu_{k_{n}}$, and $\mu=\eta_{s}$, and

$$
-\Delta_{p} v=f(1+g(v))^{p-1}+\eta_{s} \quad \text { in } \mathcal{D}^{\prime}(\Omega) ;
$$

hence in the renormalized sense; and $\mu_{k_{n}}$ converges to $\eta_{s}$ in the narrow topology.

General case. From [24], there exists $m \in \mathcal{M}_{0}(\Omega)$ and $\eta \in \mathcal{M}_{b}^{+}(\Omega)$ such that $M=m+\eta$, and there exists a sequence $\left(k_{n}\right)$ tending to $\infty$, such that there exists $M_{k_{n}} \in W^{-1, p^{\prime}}(\Omega) \cap \mathcal{M}_{b}(\Omega)$ such that $-\Delta_{p} T_{k_{n}}(v)=M_{k_{n}}$ in $\mathcal{D}^{\prime}(\Omega)$, and $\eta_{k_{n}}=M_{k_{n}\left\llcorner\left\{v=k_{n}\right\}\right.} \in \mathcal{M}_{0}^{+}(\Omega)$ and $M_{k_{n}}=m\left\llcorner\left\{v<k_{n}\right\}+\eta_{k_{n}}\right.$, and $\left(\eta_{k_{n}}\right)$ converges weakly* to $M$; and for any for any $h \in W^{1, \infty}(\mathbb{R})$ such that $h^{\prime}$ has a compact support, and any $\varphi \in \mathcal{D}(\Omega)$

$$
\int_{\Omega}|\nabla v|^{p-2} \nabla v \cdot \nabla(h(v) \varphi) d x=\int_{\Omega} h(v) \varphi d m+h(\infty) \int_{\Omega} \varphi d \eta .
$$

But $M_{k_{n}}=m\left\llcorner\left\{v<k_{n}\right\}+\eta_{k_{n}}=\phi \chi_{\left\{v<k_{n}\right\}}+\mu_{k_{n}}\right.$, hence $\eta_{k_{n}}=\mu_{k_{n}}$ and $m\left\llcorner\left\{v<k_{n}\right\}=\phi \chi_{\left\{v<k_{n}\right\}}\right.$, and $\{v=\infty\}$ is of capacity 0 , thus $m=\phi$, and $\mu=\eta$, thus (1.13) holds.

Moreover if $\Lambda<\infty$, then $L<\infty$, and $u, v \in W_{0}^{1, p}(\Omega) \cap L^{\infty}(\Omega)$, and $\mu=\alpha=0$, and $u, v$ are variational solution of $(\mathrm{PU} \lambda)$, and $(\mathrm{PV} \lambda)$. If $g$ is bounded, in particular if $L=\Lambda=\infty$ and $\beta \in L^{1}((0, \infty))$, then $\mu=e^{\gamma(\infty)} \alpha_{s}$, thus $\mu$ is singular; and $\mu_{k}$ converges in the narrow topology, thus $v$ is a renormalized solution of (1.11). If $\Lambda=\infty$ and $\beta \notin L^{1}((0, \infty))$, then $\alpha_{s}=0$ from (3.6). 


\section{The case $\beta$ constant, g linear}

We begin by the case of problems (1.4) and (1.5), where $f \not \equiv 0$, and

$$
\beta(u) \equiv p-1, \text { or equivalently } g(v)=v,
$$

where the eigenvalue $\lambda_{1}(f)$ defined at (1.16) is involved.

\subsection{Some properties of $\lambda_{1}(f)$}

(i) Let $f \in L^{1}(\Omega), f \geqq 0, f \not \equiv 0$, such that $\lambda_{1}(f)>0$. Let $C>0$. Then for any $\varepsilon>0$, there exists $K_{\varepsilon}=K_{\varepsilon}(\varepsilon, p, C)>0$ such that for any $v, w \in W_{0}^{1, p}(\Omega)$,

$$
\begin{aligned}
\int_{\Omega} f(C+|v|)^{p} d x \leqq(1 & +\varepsilon) \int_{\Omega} f|v|^{p} d x+K_{\varepsilon} \leqq \frac{1+\varepsilon}{\lambda_{1}(f)}\|\nabla v\|_{L^{p}(\Omega)}^{p}+K_{\varepsilon} \\
\int_{\Omega} f(C+|v|)^{p-1}|w| d x & \leqq\left(\int_{\Omega} f(C+|v|)^{p} d x\right)^{1 / p^{\prime}}\left(\int_{\Omega} f|w|^{p} d x\right)^{1 / p} \\
& \leqq \frac{1}{\lambda_{1}(f)^{1 / p}}\left(\int_{\Omega} f(C+|v|)^{p} d x\right)^{1 / p^{\prime}}\|\nabla w\|_{L^{p}(\Omega)}
\end{aligned}
$$

Thus $f(C+|v|)^{p-1} \in W^{-1, p^{\prime}}(\Omega) \cap L^{1}(\Omega)$, in particular $f \in W^{-1, p^{\prime}}(\Omega)$, and with new $\varepsilon$ and $K_{\varepsilon}$,

$$
\left\|f(C+|v|)^{p-1}\right\|_{W^{-1, p^{\prime}}(\Omega)} \leqq \frac{1+\varepsilon}{\lambda_{1}(f)}\|\nabla v\|_{L^{p}(\Omega)}^{p-1}+K_{\varepsilon} .
$$

(ii) If $f \in L^{r}(\Omega)$, with $r \geqq N / p>1$, or $r>1=N / p$, then $\lambda_{1}(f)>0$, and $\lambda_{1}(f)$ is attained at some first nonnegative eigenfunction $\phi_{1} \in W_{0}^{1, p}(\Omega)$ of problem (1.15), from [48]. If $r>N / p$, then $\phi_{1} \in L^{\infty}(\Omega)$, from Proposition 2.14, and $\phi_{1}$ is locally Hölder continuous, from [21]. If $r=N / p>1$, then $\phi_{1} \in L^{k}(\Omega)$ for any $k \geqq 1$.

(iii) If $0 \in \Omega, p<N$ and $f(x)=1 /|x|^{p}$, then $f \notin L^{N / p}(\Omega)$, but $\lambda_{1}(f)>0$ from the Hardy inequality, given by $\lambda_{1}(f)=((N-p) / p)^{p}$ and $\lambda_{1}(f)$ is not attained.

\subsection{Proof of Theorem 1.2}

Theorem 1.2 is a consequence of Theorem 1.1 and of the two following results. The first one is relative to the case without measure:

Theorem 4.1 If $\lambda>\lambda_{1}(f) \geqq 0$, or $\lambda=\lambda_{1}(f)>0$ and $f \in L^{N / p}(\Omega), p<N$, then problem

$$
-\Delta_{p} v=\lambda f(1+v)^{p-1} \quad \text { in } \Omega, \quad v=0 \quad \text { on } \partial \Omega .
$$

admits no renormalized solution, and problem (1.4) has no solution. If $0<\lambda<\lambda_{1}(f)$ there exists a unique positive solution $v \in W_{0}^{1, p}(\Omega)$. If moreover $f \in L^{r}(\Omega), r>N / p$, then $v \in L^{\infty}(\Omega)$. If $f \in L^{N / p}(\Omega), p<N$, then $v \in L^{k}(\Omega)$ for any $k>1$. 
Proof. (i) If (4.4) has a solution then also problem (1.4) has a solution $u \in \mathcal{W}$, from Theorem 1.1. And $u \in W_{0}^{1, p}(\Omega)$ from Remark 3.2. Taking $\varphi=\psi^{p}$ with $\psi \in \mathcal{D}^{\prime}(\Omega), \psi \geqq 0$ as a test function we obtain

$$
\begin{aligned}
(p-1) \int_{\Omega}|\nabla u|^{p} \psi^{p} d x+\lambda \int_{\Omega} f \psi^{p} d x & =p \int_{\Omega} \psi^{p-1}|\nabla u|^{p-2} \nabla u . \nabla \psi d x \\
& \leqq \int_{\Omega}|\nabla \psi|^{p} d x+(p-1) \int_{\Omega}|\nabla u|^{p} \psi^{p} d x
\end{aligned}
$$

then from the Young inequality,

$$
\lambda \int_{\Omega} f \psi^{p} d x \leqq \int_{\Omega}|\nabla \psi|^{p} d x
$$

by density we obtain that $\lambda \leqq \lambda_{1}(f)$. In particular if $\lambda_{1}(f)=0$ there is no solution for $\lambda>0$.

(ii) Assume $\lambda=\lambda_{1}(f)>0$ and $f \in L^{N / p}(\Omega), p<N$. Taking an eigenfunction $\phi_{1} \in W_{0}^{1, p}(\Omega)$ as above, we get

$$
\int_{\Omega}\left|\nabla \phi_{1}\right|^{p} d x=\lambda_{1}(f) \int_{\Omega} f \phi_{1}^{p} d x
$$

Consider a sequence of nonnegative functions $\psi_{n} \in \mathcal{D}(\Omega)$ converging to $\phi_{1}$ strongly in $W_{0}^{1, p}(\Omega)$. Taking $\psi_{n}^{p} \in W_{0}^{1, p}(\Omega) \cap L^{\infty}(\Omega)$ as a test function, we find

$$
(p-1) \int_{\Omega}|\nabla u|^{p} \psi_{n}^{p} d x+\lambda_{1}(f) \int_{\Omega} f \psi_{n}^{p-1} d x=p \int_{\Omega} \psi_{n}^{p-1}|\nabla u|^{p-2} \nabla u . \nabla \psi_{n} d x .
$$

For any function $\phi \in W_{0}^{1, p}(\Omega)$, we set

$$
\begin{gathered}
L(u, \phi):=(p-1)|\nabla u|^{p} \phi^{p}+|\nabla \phi|^{p}-p \phi^{p-1}|\nabla u|^{p-2} \nabla u . \nabla \phi, \\
L_{1}(u, \phi):=(p-1)|\nabla u|^{p} \phi^{p}+|\nabla \phi|^{p}-p \phi^{p-1}|\nabla u|^{p-1}|\nabla \phi| .
\end{gathered}
$$

Thus $0 \leqq L_{1}(u, \phi) \leqq L(u, \phi)$. From (4.6),

$$
\int_{\Omega} L_{1}\left(u, \psi_{n}\right) d x+\lambda_{1}(f) \int_{\Omega} f \psi_{n}^{p} d x \leqq \int_{\Omega} L\left(u, \psi_{n}\right) d x+\lambda_{1}(f) \int_{\Omega} f \psi_{n}^{p} d x=\int_{\Omega}\left|\nabla \psi_{n}\right|^{p} d x
$$

then from the Fatou Lemma applied to a subsequence,

$$
\int_{\Omega} L_{1}\left(u, \phi_{1}\right) d x+\lambda_{1}(f) \int_{\Omega} f \phi_{1}^{p} d x \leqq \int_{\Omega} L\left(u, \phi_{1}\right) d x+\lambda_{1}(f) \int_{\Omega} f \phi_{1}^{p} d x=\int_{\Omega}\left|\nabla \phi_{1}\right|^{p} d x,
$$

hence from (4.5), we obtain $L_{1}\left(u, \phi_{1}\right)=L\left(u, \phi_{1}\right)=0$ a.e. in $\Omega$. Then

$$
\phi_{1}|\nabla u|=(p-1)\left|\nabla \phi_{1}\right|, \text { and }|\nabla u|^{p-2} \nabla u . \nabla \phi_{1}=|\nabla u|^{p-1}\left|\nabla \phi_{1}\right| \quad \text { a.e. in } \Omega \text {, }
$$




$$
\nabla u=(p-1) \frac{\nabla \phi_{1}}{\phi_{1}}=\nabla\left(\ln \left(\phi_{1}^{p-1}\right)\right) \quad \text { a.e. in } \Omega
$$

then $u=\ln \left(\phi_{1}^{p-1}\right)+k$, or $\phi_{1}^{p-1}=e^{u-k} \geqq e^{-k}$ a.e. in $\Omega$, which is contradictory.

(iii) Assume that $0<\lambda<\lambda_{1}(f)$. Then $f \in W^{-1, p^{\prime}}(\Omega)$ from above, thus $v_{1}=\mathcal{G}(\lambda f) \in W_{0}^{1, p}(\Omega)$ and $v_{1} \geqq 0$, see Remark 2.10, and $f\left(1+v_{1}\right)^{p-1} \in W^{-1, p^{\prime}}(\Omega)$. By induction we define $v_{n}=$ $\mathcal{G}\left(\lambda f\left(v_{n-1}+1\right)^{p-1} \in W_{0}^{1, p}(\Omega)\right.$, then

$$
-\Delta_{p} v_{n}=\lambda f\left(v_{n-1}+1\right)^{p-1} \quad \text { in } W^{-1, p^{\prime}}(\Omega) .
$$

Taking $v_{n}$ as test function in (4.7), then from (4.1),

$$
\int_{\Omega}\left|\nabla v_{n}\right|^{p} d x=\lambda \int_{\Omega} f\left(v_{n-1}+1\right)^{p-1} v_{n} d x \leqq \lambda \int_{\Omega} f\left(v_{n}+1\right)^{p} d x \leqq \frac{\lambda(1+\varepsilon)}{\lambda_{1}(f)} \int_{\Omega}\left|\nabla v_{n}\right|^{p} d x+\lambda K_{\varepsilon} .
$$

Taking $\varepsilon>0$ small enough, it follows that $\left(v_{n}\right)$ is bounded in $W_{0}^{1, p}(\Omega)$. The sequence is nondecreasing, thus it converges weakly in $W_{0}^{1, p}(\Omega)$, and a.e. in $\Omega$ to $v=\sup v_{n}$. For any $w \in W_{0}^{1, p}(\Omega)$, $\left|f\left(v_{n-1}+1\right)^{p-1} w\right| \leqq f(1+v)^{p-1}|w|=h$ and $h \in L^{1}(\Omega)$ from (4.2), thus $f\left(v_{n-1}+1\right)^{p-1}$ converges to $f(1+v)^{p-1}$ weakly in $W^{-1, p^{\prime}}(\Omega)$. Then $v$ is solution of (4.4), by compacity of $\left(-\Delta_{p}\right)^{-1}$, see [56]. The regularity follows from Proposition 2.14 (iii).

Uniqueness is based on Lemma 2.12, Let $v, \hat{v} \in W_{0}^{1, p}(\Omega)$ be two nonnegative solutions. Then $v \not \equiv 0$ and $\hat{v} \not \equiv 0$ since $f \not \equiv 0$. Since $-\Delta_{p} v \in W^{-1, p^{\prime}}(\Omega) \cap L^{1}(\Omega)$ and $\left(-\Delta_{p} v\right) v \geqq 0$, we obtain $\int_{\Omega}\left(-\Delta_{p} v\right) v d x=\int_{\Omega}|\nabla v|^{p} d x$, hence

$$
\int_{\Omega}\left(\frac{-\Delta_{p} v}{v^{p-1}}+\frac{\Delta_{p} \hat{v}}{\hat{v}^{p-1}}\right) v^{p} d x \geqq 0 ; \quad \int_{\Omega}\left(-\frac{\Delta_{p} \hat{v}}{\hat{v}^{p-1}}+\frac{-\Delta_{p} v}{v^{p-1}}\right) \hat{v}^{p-1} d x \geqq 0 ;
$$

but

$$
\int_{\Omega}\left(\frac{-\Delta_{p} v}{v^{p-1}}+\frac{\Delta_{p} \hat{v}}{\hat{v}^{p-1}}\right)\left(v^{p}-\hat{v}^{p}\right) d x=\lambda \int_{\Omega} f\left(\left(1+\frac{1}{v}\right)^{p-1}-\left(1+\frac{1}{\hat{v}}\right)^{p-1}\right)\left(v^{p}-\hat{v}^{p}\right) d x \leqq 0 ;
$$

then the two integrals in (4.8) are zero, hence

$$
\int_{\Omega}\left(|\nabla v|^{p}-p \frac{\hat{v}^{p-1}}{v^{p-1}}|\nabla \hat{v}|^{p-2} \nabla \hat{v} \cdot \nabla v+(p-1)|\nabla \hat{v}|^{p} \frac{\hat{v}^{p}}{v^{p}}\right) d x=0,
$$

thus $v=k \hat{v}$ for some $k>0$. Then $f\left((1+k v)^{p-1}-(k+k v)^{p-1}\right)=0$ a.e. in $\Omega$, thus $k=1$.

The second result is valid for measures which are not necessarily singular; it extends [2, Theorem $2.6]$ relative to $p=2$. The proof of a more general result will be given at Theorem 6.2.

Theorem 4.2 If $0<\lambda<\lambda_{1}(f)$, for any measure $\mu \in \mathcal{M}_{b}^{+}(\Omega)$, there exists at least one renormalized solution $v \geqq 0$ of problem

$$
-\Delta_{p} v=\lambda f(1+v)^{p-1}+\mu \quad \text { in } \Omega, \quad v=0 \quad \text { on } \partial \Omega .
$$




\section{Problem $(\mathrm{PV} \lambda)$ without measures}

Next we study problem (PV $\lambda)$ for a general function $g$.

\subsection{The range of existence for $\lambda$}

The existence of solutions of (PV $\lambda$ ) depends on the assumptions on $g$ and $f$ and the value of $\lambda$. We will sometimes make growth assumptions on $g$ of the form (1.3) for some $Q>0$ and then our assumptions on $f$ will depend on $Q$.

We begin by a simple existence result, where $g$ only satisfies (1.2), $\Lambda \leqq \infty$, with no growth condition, under a weak assumption on $f$, satisfied in particular when $f \in L^{r}(\Omega), r>N / p$.

Proposition 5.1 Assume (1.2) and $\mathcal{G}(f) \in L^{\infty}(\Omega)$. Then for $\lambda>0$ small enough, problem (PV $\left.\lambda\right)$ has a minimal bounded solution $\underline{v}_{\lambda}$ such that $\|v\|_{L^{\infty}(\Omega)}<\Lambda$.

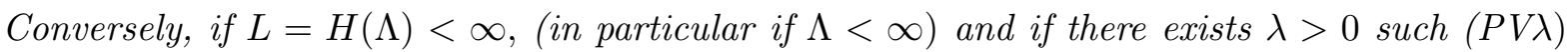
has a renormalized solution, then $\mathcal{G}(f) \in L^{\infty}(\Omega)$.

Proof. Let $w=\mathcal{G}(f) \in W_{0}^{1, p}(\Omega) \cap L^{\infty}(\Omega)$. Let $a>0$ such that $a\|w\|_{L^{\infty}(\Omega)}<\Lambda$. Let $\lambda_{0}=$ $a\left(\left(1+g\left(a\|w\|_{L^{\infty}(\Omega)}\right)\right)\right)^{-(p-1)}$ and $\lambda \leqq \lambda_{0}$ be fixed. Then

$$
-\Delta_{p}(a w)=a f(x)=\lambda_{0}\left(\left(1+g\left(a\|w\|_{L^{\infty}(\Omega)}\right)\right)\right)^{(p-1)} \geqq \lambda(1+g(a w))^{p-1}
$$

since $g$ is nondecreasing. Between the subsolution 0 and the supersolution $a w$, there exists a minimal solution $\underline{v}_{\lambda}$ obtained as the nondecreasing limit of the iterative scheme

$$
v_{n}=\mathcal{G}\left(\lambda f(x)\left(1+g\left(v_{n-1}\right)\right)^{p-1}\right), n \geqq 1 .
$$

Then $\left\|\underline{v}_{\lambda}\right\|_{L^{\infty}(\Omega)} \leqq a\|w\|_{L^{\infty}(\Omega)}<\Lambda$.

Conversely, let $v$ be a solution of $(\operatorname{PV} \lambda)$. Then $u=H(v)$ is a solution of $(\operatorname{PU} \lambda)$ and $L \geqq u \geqq$ $\lambda^{1 /(p-1)} \mathcal{G}(f)$ a.e. in $\Omega$, hence $\mathcal{G}(f) \in L^{\infty}(\Omega)$.

Remark 5.2 The converse result is sharp. Take $f=1 /|x|^{p}$ with $0 \in \Omega$, then $\mathcal{G}(f) \notin L^{\infty}(\Omega)$. Hence if $L<\infty$ there is no solution of $(P V \lambda)$ for any $\lambda>0$; for example, there is no solution of problem

$$
-\Delta_{p} v=\frac{\lambda}{|x|^{p}}(1+v)^{Q} \quad \text { in } \Omega, \quad v=0 \quad \text { on } \partial \Omega .
$$

for $Q>p-1$. Otherwise from Theorem 1.2, for $Q=p-1$ and $0<\lambda<\lambda_{1}(f)$, there exists a solution; in that case $H(\infty)=\infty$. 
Remark 5.3 When $\Lambda<\infty$, and $g$ has an asymptote at $\Lambda$, it may exist solutions with $\|v\|_{L^{\infty}(\Omega)}=\Lambda$. Consider example 9 of paragraph 3.2 with $p=2$ and $\Omega=B(0,1)$. Here $1+g(v)=(1-v)^{-Q}, Q>0$, and $\beta(u)=Q(1-(Q+1) u)$. For $\lambda=2((N-2) Q+N) /(Q+1)^{2}$, problem $(P U \lambda)$ admits the solution $u=\left(1-r^{2}\right) /(Q+1)$. Then $v=\Psi(u)=1-r^{2 /(Q+1)} \in W_{0}^{1,2}(\Omega)$, and $\|v\|_{L^{\infty}(\Omega)}=1$.

The range of $\lambda \geqq 0$ for which problem $(\mathrm{PV} \lambda)$ has a solution depends a priori on the regularity of the solutions. We introduce three classes of solutions. In case $\Lambda<\infty$ the notion of solution includes the fact that $0 \leqq v(x)<\Lambda$ a.e. in $\Omega$.

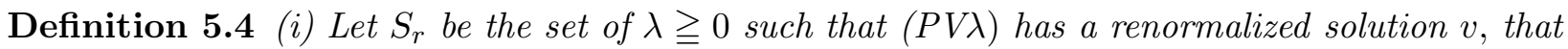
means $w \in \mathcal{W}$.

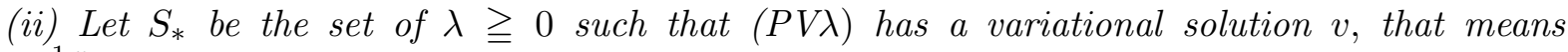
$v \in W_{0}^{1, p}(\Omega)$.

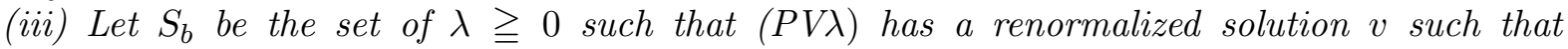
$\|v\|_{L^{\infty}(\Omega)}<\Lambda$.

Remark 5.5 The sets $S_{r}, S_{*}, S_{b}$ are intervals:

$$
S_{r}=\left[0, \lambda_{r}\right), \quad S_{*}=\left[0, \lambda^{*}\right), \quad S_{b}=\left[0, \lambda_{b}\right) \quad \text { with } \lambda_{b} \leqq \lambda^{*} \leqq \lambda_{r} \leqq \infty .
$$

Indeed if $\lambda_{0}$ belongs to some of these sets, and $v_{\lambda_{0}}$ is a solution of $\left(P V \lambda_{0}\right)$, then $v_{\lambda_{0}}$ is a supersolution of $(P V \lambda)$ for any $\lambda<\lambda_{0}$. Between the subsolution 0 and $v_{\lambda_{0}}$, there exists a minimal solution of $(P V \lambda)$, obtained as the nondecreasing limit of the iterative scheme (5.1).

In case $\Lambda=\infty, S_{b}$ is the set of of $\lambda \geqq 0$ such that $(P V \lambda)$ has a solution $v \in W_{0}^{1, p}(\Omega) \cap L^{\infty}(\Omega)$. For any $\lambda<\lambda_{b}$ there exists a minimal bounded solution $\underline{v}_{\lambda}$. And $\lambda_{b} \leqq \lambda^{*}$ since any renormalized bounded solution is in $W_{0}^{1, p}(\Omega)$ from Remark 2.5.

In case $\Lambda<\infty$, then $\lambda_{r}=\lambda^{*}$, since $S_{r}=S_{*}$, from Remark 2.5. Moreover $\lambda^{*}<\infty$. Indeed any solution $v$ of $(P V \lambda)$ satisfies $\lambda \mathcal{G}(f) \leqq v<\Lambda$ a.e. in $\Omega$, and $\mathcal{G}(f) \not \equiv 0$.

A main question is to know if $\lambda_{b}=\lambda^{*}=\lambda_{r}$, as it is the case when $g(v)=v$, from Theorem 1.2, where $\lambda^{*}=\lambda_{1}(f)$. It was shown when $g$ is defined on $[0, \infty)$ and convex in [15] for $p=2$. The method used was precisely based on the transformation $u=H(v)$, even if problem (PU $\lambda$ ) was not introduced. By using the equations satisfied by truncations as in the proof of Theorem 1.1, we can extend the kea point of the proof:

Theorem 5.6 Let $g_{1}, g_{2} \in C^{1}([0, \Lambda))$ be nondecreasing, with $0<g_{2} \leqq g_{1}$ on $[0, \Lambda)$. Let $v \in \mathcal{W}(\Omega)$ such that $-\Delta_{p} v \geqq 0$ a.e. in $\Omega$, and $0 \leqq v<\Lambda$ a.e. in $\Omega$. Set

$$
H_{1}(\tau)=\int_{0}^{\tau} \frac{d s}{g_{1}(s)}, \quad H_{2}(\tau)=\int_{0}^{\tau} \frac{d s}{g_{2}(s)}
$$


Assume that

$$
0 \leqq g_{2}^{\prime} \circ H_{2}^{-1} \leqq g_{1}^{\prime} \circ H_{1}^{-1} \quad \text { on }\left[0, H_{1}(\Lambda)\right)
$$

Then $\bar{v}=H_{2}^{-1}\left(H_{1}(v)\right) \in \mathcal{W}$, and $\bar{v} \leqq v$, and

$$
-\Delta_{p} \bar{v} \geqq\left(\frac{g_{2}(\bar{v})}{g_{1}(v)}\right)^{p-1}\left(-\Delta_{p} v\right) \quad \text { in } L^{1}(\Omega) .
$$

Proof. We can assume that $g_{1}(0)=1$. Let $u=H_{1}(v)$, and $F=-\Delta_{p} v$. Applying Theorem 1.1 with $g=g_{1}-1$ and $f=F g_{1}(v)^{1-p} \leqq F$, the function $u$ is a renormalized solution of

$$
-\Delta_{p} u=\beta_{1}(u)|\nabla u|^{p}+F g_{1}(v)^{1-p} \quad \text { in } \Omega, \quad u=0 \quad \text { on } \partial \Omega,
$$

where $\beta_{1}(u)=(p-1) g_{1}^{\prime}(v)=(p-1) g_{1}^{\prime}\left(H_{1}^{-1}(u)\right)$. Let

$$
\bar{v}=H_{2}^{-1}(u)=\left(H_{2}^{-1} \circ H_{1}\right)(v)
$$

then $\bar{v} \leqq v$, because $g_{2} \leqq g_{1}$. Moreover $g_{1}^{\prime}(v) \geqq g_{2}^{\prime}(\bar{v})$, thus we can write

$$
\beta_{1}(u)|\nabla u|^{p}=(p-1) g_{2}^{\prime}(\bar{v})|\nabla u|^{p}+\eta=\beta_{2}(u)|\nabla u|^{p}+\eta,
$$

with $\eta \in L^{1}(\Omega), \eta \geqq 0$; thus

$$
-\Delta_{p} u=\beta_{2}(u)|\nabla u|^{p}+\bar{f}
$$

with $\bar{f}=F g_{1}(v)^{1-p}+\eta$. From Lemma 3.4, the truncations $T_{k}(v), T_{K}(u), T_{k}(\bar{v})$ satisfy respectively

$$
\begin{aligned}
-\Delta_{p} T_{k}(v) & =F \chi_{\{v \leqq k\}}+\mu_{k}, \\
-\Delta_{p} T_{K}(u) & =\beta_{1}\left(T_{K}(u)\right)\left|\nabla T_{K}(u)\right|^{p}+F g_{1}(v)^{1-p} \chi\{u \leqq K\}+\alpha_{K}, \\
-\Delta_{p} T_{k}(\bar{v}) & =\bar{f} g_{2}(\bar{v})^{p-1} \chi_{\{\bar{v} \leqq k\}}+\bar{\mu}_{k},
\end{aligned}
$$

in $\mathcal{D}^{\prime}(\Omega)$, where

$$
\alpha_{K}=g_{1}(v)^{1-p} \mu_{k}, \quad \bar{\mu}_{k}=\left(g_{2}(k) / g_{1}(k)\right)^{p-1} \mu_{k} .
$$

As in the proof of Theorem 1.1, we obtain $\bar{f} g_{2}(\bar{v})^{p-1} \in L^{1}(\Omega)$, and $\bar{f} g_{2}(\bar{v})^{p-1} \chi_{\{\bar{v}<k\}}$ converges to $\bar{f} g_{2}(\bar{v})^{p-1}$ strongly in $L^{1}(\Omega)$. Moreover $\mu_{k}$ converges to 0 in the narrow topologyas $k \rightarrow \Lambda$, thus $\lim \mu_{k}(\Omega)=0$; and $g_{2}(k) \leqq g_{1}(k)$, thus $\lim \bar{\mu}_{k}(\Omega)=0$, and $\bar{\mu}_{k}$ converges to 0 in the narrow topology. Then from Theorem 2.6, $\bar{v}$ is a renormalized solution of

$$
-\Delta_{p} \bar{v}=\bar{f} g_{2}(\bar{v})^{p-1} \quad \text { in } \Omega, \quad \bar{v}=0 \quad \text { on } \partial \Omega .
$$

Then $-\Delta_{p} \bar{v} \in L^{1}(\Omega)$, and $\bar{v}$ satisfies (5.4). 
Remark 5.7 Assumption (5.3) is equivalent to the concavity of the function $\bar{\phi}=H_{2}^{-1} \circ H_{1}$; and (5.4) means that

$$
-\Delta_{p} \bar{\phi}(v) \geqq\left(\bar{\Phi}^{\prime}(v)\right)^{p-1}\left(-\Delta_{p} v\right) \quad \text { in } L^{1}(\Omega) .
$$

If we take any concave function $\phi$ this inequality is formal. For the particular choice $\phi=\bar{\phi}$, the inequality is not formal, since no measure appears.

Our main result covers in particular Theorem 1.3. Some convexity assumptions are weakened:

Theorem 5.8 Let $g$ satisfying (1.2), and $H$ be defined by (3.2) on $[0, \Lambda)$, and $f \in L^{1}(\Omega)$. In case $\Lambda=\infty, L=H(\Lambda)=\infty$ we suppose $f \in L^{r}(\Omega), r>N / p$. Assume that for some $\lambda>0$ there exists a renormalized solution $v$ of

$$
-\Delta_{p} v=\lambda f(x)(1+g(v))^{p-1} \quad \text { in } \Omega, \quad v=0 \quad \text { on } \partial \Omega
$$

such that $0 \leqq v(x)<\Lambda$ a.e. in $\Omega$.

(i) Suppose that $H \times(1+g)$ is convex on $[0, \Lambda)$, or that $g$ is convex near $\Lambda$. Then for any $\varepsilon \in(0,1)$ there exists a bounded solution $w$, such that $\|w\|_{L^{\infty}(\Omega)}<\Lambda$ of

$$
-\Delta_{p} w=\lambda(1-\varepsilon)^{p-1} f(x)(1+g(w))^{p-1} \quad \text { in } \Omega, \quad w=0 \quad \text { on } \partial \Omega .
$$

In other words, $\lambda_{b}=\lambda^{*}=\lambda_{r}$.

(ii) Suppose that $g$ is convex on $[0, \Lambda)$. Then for any $\varepsilon \in(0,1)$ there exists a bounded solution $w$ such that $\|w\|_{L^{\infty}(\Omega)}<\Lambda$ of

$$
-\Delta_{p} w=\lambda f(x)(1+g(w)-\varepsilon)^{p-1} \quad \text { in } \Omega, \quad w=0 \quad \text { on } \partial \Omega .
$$

In particular if $\lambda^{*}<\infty$, for any $c>0$, there exists no solution of problem

$$
-\Delta_{p} v=\lambda^{*} f(x)(1+g(v)+c)^{p-1} \quad \text { in } \Omega, \quad v=0 \quad \text { on } \partial \Omega .
$$

Proof. (i) First case: $L=H(\Lambda)=\int_{0}^{\Lambda} d s /(1+g(s))<\infty$.

- First suppose $H \times(1+g)$ convex on $[0, \Lambda)$. We take $g_{1}=1+g$ and $g_{2}=(1-\varepsilon) g_{1}$. Then

$$
H_{2}=H /(1-\varepsilon), \quad H_{2}^{-1}(u)=H^{-1}((1-\varepsilon) u)=\Psi((1-\varepsilon) u) .
$$

Condition (5.3) is equivalent to $(1-\varepsilon) u g^{\prime}\left(\Psi\left((1-\varepsilon)^{1 /(p-1)} u\right) \leqq u g^{\prime}(\Psi(u))\right.$. In terms of $u$, it means that the function $u \longmapsto u \beta(u)$ is non decreasing; in terms of $v$ it means that $H \times g^{\prime}$ is nondecreasing. This is true when $H \times(1+g)$ is convex, since $(H \times(1+g))^{\prime}=1+H \times g^{\prime}$. Then from Proposition 5.6, the function $\bar{v}=\Psi((1-\varepsilon) H(v))$ satisfies

$$
-\Delta_{p} \bar{v} \geqq \lambda(1-\varepsilon)^{p-1} f(x)(1+g(\bar{v}))^{p-1} .
$$


Thus there exists a solution $w$ of (5.5) such that $w \leqq \bar{v}$. And $\bar{v}(x) \leqq \Psi((1-\varepsilon) L)<\Lambda$ a.e. in $\Omega$, hence $w$ is bounded, and moreover $\|w\|_{L^{\infty}(\Omega)}<\Lambda$.

- Next suppose $g$ convex on $[A, \Lambda)$, with $0 \leqq A<\Lambda$. Let $M=1+g(A)$. Taking $\varepsilon>0$ small enough, we construct a convex nondecreasing function $g_{1} \in C^{1}([0, \Lambda))$ such that $g_{1} \geqq 1+g$, and

$$
g_{1}(s)=M \text { on }[0, A-c], \quad g_{1}(s) \leqq M(1+2 \varepsilon) \text { on }[0, A+d], \quad g_{1}(s)=1+g(s) \text { on }[A+d, \infty),
$$

with $c=2 \varepsilon M$ and $d \leqq 2 \varepsilon M g^{\prime}(A)$ : we use a portion of circle tangent to the graph of $1+g$ and to the line of ordinate $M$; in case $g^{\prime}(A)=0$ we take $\left.g_{1}=1+g\right)$. We set $g_{2}=(1-\varepsilon) g_{1}$. The function $\bar{v}=H_{2}^{-1}\left(H_{1}(v)\right)=\Psi_{1}\left((1-\varepsilon) H_{1}(v)\right)$ satisfies

$$
-\Delta_{p} \bar{v} \geqq \lambda f(x) F_{\varepsilon}^{p-1}, \quad \text { where } F_{\varepsilon}=(1-\varepsilon) \frac{g_{1}(\bar{v})}{g_{1}(v)}(1+g(v)),
$$

and $\bar{v} \leqq v$. On the set $\{\bar{v} \leqq v \leqq A+d\}$, we find $M \leqq g_{1}(\bar{v})$ and $g_{1}(v) \leqq M(1+2 \varepsilon)$, thus $F_{\varepsilon} \geqq$ $(1-3 \varepsilon)(1+g(\bar{v}))$. On the set $\{A+d \leqq \bar{v} \leqq v\}$, we get $g_{1}(\bar{v})=g_{1}(v)=1+g(v)$, thus $F_{\varepsilon} \geqq$ $(1-\varepsilon)(1+g(\bar{v}))$. On the set $\{\bar{v} \leqq A+d \leqq v\}$, there holds $M \leqq g_{1}(\bar{v}) \leqq M(1+2 \varepsilon) \leqq 1+g(v)$, thus again $F_{\varepsilon} \geqq(1-\varepsilon)(1+g(\bar{v}))$. Then again $-\Delta_{p} \bar{v} \geqq \lambda(1-\varepsilon)^{p-1} f(x)(1+g(\bar{v}))^{p-1}$, and we conclude as above by replacing $\varepsilon$ by $3 \varepsilon$.

Second case: $L=\infty$. Here $\bar{v}$ can be unbounded. Extending [15, we perform a bootstrapp based on Lemma 2.13. The function $H_{1}$ is concave, thus

$$
\left.H_{1}(v)-H_{1}(\bar{v}) \leqq(v-\bar{v}) H_{1}^{\prime}(\bar{v})\right)=\frac{v-\bar{v}}{g_{1}(\bar{v})} \leqq \frac{v}{g_{1}(\bar{v})}
$$

and $H_{1}(\bar{v})=(1-\varepsilon) H_{1}(v)$, hence $\varepsilon(1+g(\bar{v})) \leqq \varepsilon g_{1}(\bar{v}) \leqq v / H_{1}(v) \leqq C(1+v)$ for some $C>0$. Then $(1+g(w))^{p-1} \in L^{\sigma}(\Omega)$ for any $\sigma \in[1, N /(N-p))$. Since $f \in L^{r}(\Omega), r>N / p$, from Hölder inequality, there exists $m_{1}>1$ such that $f g(w)^{p-1} \in L^{m_{1}}(\Omega)$. If $p=N$, then $\bar{v} \in L^{\infty}(\Omega)$ from Lemma 2.13 and we conclude as above. Next assume $p<N$. We can suppose $m_{1}<N / p$. Setting $w_{1}=w, w_{1}$ is a solution of $\left(\operatorname{PV}(1-\varepsilon)^{p-1} \lambda\right)$, and $-\Delta_{p} w_{1} \in L^{m_{1}}(\Omega)$; from Lemma 2.13, $w_{1}^{s} \in L^{1}(\Omega)$ with $s=(p-1) N m_{1} /\left(N-p m_{1}\right)$. Replacing $1+g$ by $(1-\varepsilon)(1+g)$ we construct in the same way a solution $w_{2}$ of $\left(\mathrm{PV}(1-\varepsilon)^{2(p-1)} \lambda\right)$ such that $\left.g\left(w_{2}\right) \leqq C\left(1+w_{1}\right)\right)$, By induction we construct a solution $w_{n}$ of $\left(\mathrm{PV}(1-\varepsilon)^{n(p-1)} \lambda\right)$ such that $\left.g\left(w_{n}\right) \leqq C\left(1+w_{n-1}\right)\right)$, thus $f g\left(w_{n}\right)^{p-1} \in L^{m_{n}}(\Omega)$, with $1 / m_{n}-1 / r=1 / m_{n-1}-p / N$. There exists a finite $\bar{n}=\bar{n}(r, p, N)$ such that $m_{\bar{n}}>N / p$, thus $w_{\bar{n}+1} \in L^{\infty}(\Omega)$ from Lemma 2.13. Since $\varepsilon$ is arbitrary, we obtain a bounded solution of (5.5).

(ii) Suppose that $g$ is convex on $[0, \Lambda)$. We take $g_{1}=1+g$ and $g_{2}=g_{1}-\varepsilon$, then (5.3) is satisfied, because $g^{\prime}$ is nondecreasing and $H_{1} \leqq H_{2}$. Then we construct a solution $w$ of (5.6), such that $w \leqq \bar{v}=H_{2}^{-1}\left(H_{1}(v)\right)$. Here we only find $w(x) \leqq \bar{v}(x)<L$ a.e. in $\Omega$, by contradiction, but not $\|w\|_{L^{\infty}(\Omega)}<\Lambda$. As above (5.8) holds. And $H_{1}(v)=H_{2}(\bar{v})$, hence

$$
H_{1}(v)-H_{1}(\bar{v})=\varepsilon \int_{0}^{\bar{v}} \frac{d s}{g_{1}(s)\left(g_{1}(s)-\varepsilon\right)} d s \geqq \varepsilon \int_{0}^{\bar{v}} \frac{d s}{g_{1}(s)^{2}} d s
$$


Then there exists $C>0$ such that $H_{1}(v)-H_{1}(\bar{v}) \geqq C \varepsilon$, a.e. on the set $\{\bar{v}>1\}$, thus $g_{1}(\bar{v}) \leqq$ $v / \varepsilon C(A)$ on this set. Hence there exists $C_{\varepsilon}>0$ such that $\varepsilon g_{1}\left(v_{1}\right) \leqq C_{\varepsilon}(1+v)$. Replacing $g$ by $g-n \varepsilon$, in a finite number of steps as above we find a solution of (5.5), since $\varepsilon$ is arbitrary.

Assume that there exists a solution of (5.7) for some $c>0$. Then

$$
-\Delta_{p} v=\lambda^{*}(1+c)^{p-1} f(x)(1+g(v) /(1+c))^{p-1} \quad \text { in } \Omega .
$$

Considering $g /(1+c)$ and $\varepsilon=c / 2(1+c)$, there exists a bounded solution $w$ such that $\|w\|_{L^{\infty}(\Omega)}<\Lambda$, of

$$
-\Delta_{p} w=\lambda^{*} f(x)(1+g(w)+c / 2)^{p-1} \quad \text { in } \Omega,
$$

We take $\alpha>0$ small enough such that $\alpha \leqq c / 2\left(1+\|g(w)\|_{L^{\infty}(\Omega)}\right)$. Then $w$ is a supersolution of $\left(\operatorname{PV} \lambda^{*}(1+\alpha)^{p-1}\right)$, thus there exists a solution $y$ of this problem such that $\|y\|_{L^{\infty}(\Omega)}<\Lambda$, which contradicts the definition of $\lambda^{*}$.

\subsection{Cases where $g$ has a slow growth}

In the linear case $g(v)=v$, we have shown that $\lambda^{*}=\lambda_{1}(f)$. Next we consider the cases where $g$ has a slow growth, that means $g$ satisfies (1.3) for some $Q \in\left(0, Q_{1}\right)$.

First suppose that $g$ is at most linear near $\infty$ and show a variant of Theorem 4.1 ,

Corollary 5.9 Assume that $\Lambda=\infty$, and $g$ satisfies (1.3) with $Q=p-1$, that means

$$
0 \leqq M_{p-1}^{1 /(p-1)}=\varlimsup_{\tau \rightarrow \infty} \frac{g(\tau)}{\tau}<\infty,
$$

Then $\lambda^{*} \geqq M_{p-1} \lambda_{1}(f)$ : if $M_{p-1} \lambda<\lambda_{1}(f)$ there exists at least a solution $v \in W_{0}^{1, p}(\Omega)$ to problem $(P V \lambda)$; if $(1+g(v)) / v$ is decreasing, the solution is unique.

If $f \in L^{r}(\Omega), r>N / p$, any solution satisfies $v \in L^{\infty}(\Omega)$, thus $\lambda_{b}=\lambda^{*}$. If $f \in L^{N / p}(\Omega)$ and $p<N$, any solution $v \in L^{k}(\Omega)$ for any $k>1$.

Proof. Let $M>M_{p-1}$ such that $M \lambda<\lambda_{1}(f)$. There exists $A>0$ such that $(1+g(s))^{p-1} \leqq$ $M(A+s)^{p-1}$ on $[0, \infty)$. Defining $v_{1}=\mathcal{G}(\lambda f) \in W_{0}^{1, p}(\Omega)$ as in the linear case of Theorem 4.1, and $v_{n}=\mathcal{G}\left(\lambda f\left(1+g\left(v_{n-1}\right)\right)^{p-1}\right) \in W_{0}^{1, p}(\Omega)$, we find from (4.3)

$$
\int_{\Omega}\left|\nabla v_{n}\right|^{p} d x \leqq \lambda M \int_{\Omega} f\left(A+v_{n-1}\right)^{p-1} v_{n} d x \leqq \frac{\lambda M(1+\varepsilon)}{\lambda_{1}(f)} \int_{\Omega}\left|\nabla v_{n}\right|^{p} d x+\lambda K_{\varepsilon},
$$

with a new $K_{\varepsilon}>0$, and conclude as in the linear case. Uniqueness follows from Lemma 2.12, and regularity from Proposition 2.14, (iii).

Corollary 5.9 obviously applies to the case where $g$ is sublinear near $\infty$, that means $g$ satisfies (1.3) with $Q<p-1$, and shows that if $\lambda_{1}(f)>0$, then $\lambda^{*}=\infty$. In fact existence of a renormalized solution can be obtained for some functions $f$ without assuming $\lambda_{1}(f)>0$, as it was observed in [60]: 
Proposition 5.10 Assume that $p<N, \Lambda=\infty$, and $g$ satisfies (1.3) with $Q \in(0, p-1)$ and $f \in L^{r}(\Omega), r \in(1, N / p)$, with $Q r^{\prime}<Q_{1}$.

Then for any $\lambda>0$ there exists a renormalized solution $v$ of $(P V \lambda)$ such that $v^{d} \in L^{1}(\Omega)$ for $d=N r(p-1-Q) /(N-p r)$. In particular $\lambda_{r}=\infty$.

If $(Q+1) r^{\prime} \leqq p^{*}$, then $v \in W_{0}^{1, p}(\Omega)$, thus $\lambda^{*}=\infty$.

If $(Q+1) r^{\prime}>p^{*}$, then $|\nabla v|^{\theta} \in L^{1}(\Omega)$ for $\theta=N r(p-1-Q) /(N-(Q+1) r)$.

Proof. Let $M>0$ such that $(1+g(t))^{p-1} \leqq M(1+t)^{Q}$ for $t \geqq 0$. For any fixed $n \in \mathbb{N}$, there exists $v_{n} \in W_{0}^{1, p}(\Omega)$ such that

$$
-\Delta_{p} v_{n}=\lambda T_{n}\left(f(x)\left(1+g\left(v_{n}\right)\right)^{p-1}\right) .
$$

It is obtained for example as the limit of the nondecreasing iterative sheme $v_{n, k}=\mathcal{G}\left(\lambda T_{n}(f(x)(1+\right.$ $\left.\left.\left.g\left(v_{n, k-1}\right)\right)^{p-1}\right)\right), k \geqq 1, v_{n, 0}=0$. We take $\phi_{\beta}\left(v_{n}\right)$ as a test function, where $\phi_{\beta}(w)=\int_{0}^{w}(1+|t|)^{-\beta} d t$, for given real $\beta<1$. Setting $\alpha=1-\beta / p$ and $w_{n}=\left(1+v_{n}\right)^{\alpha}-1$, we get

$$
\frac{1}{\alpha^{p}} \int_{\Omega}\left|\nabla w_{n}\right|^{p} d x=\int_{\Omega} \frac{\left|\nabla v_{n}\right|^{p}}{\left(1+v_{n}\right)^{\beta}} d x \leqq(1-\beta)^{-1} \lambda M \int_{\Omega} f\left(1+v_{n}\right)^{1-\beta+Q} d x .
$$

From the Sobolev injection, There exists $C>0$ such that

$$
\left(\int_{\Omega} w_{n}^{p^{*}} d x\right)^{p / p^{*}} \leqq C \int_{\Omega} f\left(1+w_{n}\right)^{(1-\beta+Q) / \alpha} d x \leqq C\|f\|_{L^{1}(\Omega)}+C\|f\|_{L^{r}(\Omega)}\left(\int_{\Omega} w_{n}^{(1-\beta+Q) r^{\prime} / \alpha} d x\right)^{1 / r^{\prime}}
$$

Taking $\beta=\left((Q+1) r^{\prime}-p^{*}\right) /\left(r^{\prime}-N /(N-p)<1\right.$, we find $(1-\beta+Q) r^{\prime} / \alpha=p^{*}$. Then $\left(w_{n}\right)$ is bounded in $W_{0}^{1, p}(\Omega)$, thus $\left(v_{n}^{d}\right)$ is bounded in $L^{1}(\Omega)$. If $(Q+1) r^{\prime} \leqq p^{*}$ then $\beta \leqq 0$, thus $\left(v_{n}\right)$ is bounded in $W_{0}^{1, p}(\Omega)$. If $(Q+1) r^{\prime}>p^{*}$, then $\beta>0$, and

$$
\int_{\Omega}\left|\nabla v_{n}\right|^{\theta} d x \leqq\left(\int_{\Omega} \frac{\left|\nabla v_{n}\right|^{p}}{\left(1+v_{n}\right)^{\beta}} d x\right)^{1 / \theta}\left(\int_{\Omega}\left(1+v_{n}\right)^{d} d x\right)^{\beta \theta / d p}
$$

thus $\left(\left|\nabla v_{n}\right|^{\theta}\right)$ is bounded in $L^{1}(\Omega)$, where $\theta<p$. Then $\left.\left(f(x) g\left(v_{n}\right)\right)^{p-1}\right)$ is bounded in $L^{\sigma}(\Omega)$ with $\sigma=r d(r Q+d)>1$. From Remark 2.11, up to a subsequence, $\left(v_{n}\right)$ converges a.e. in $\Omega$ to a renormalized solution of the problem with the same regularity.

Remark 5.11 (i) The fact that $\lambda_{r}=\infty$ is much more general, as it will be shown at Theorem 6.2. (ii) The regularity of the solution constructed at Proposition 5.10 is a little stronger that the one exspected from Proposition (2.14) (vi). We do not know if any solution of the problem has the same regularity. 
Our next result concerns any function $g$ with a slow growth, without assumption of convexity. It is a direct consequence of Proposition 2.14;

Proposition 5.12 Assume that $\Lambda=\infty$, and $g$ satisfies (1.3) with $Q \in\left[p-1, Q_{1}\right)$ and $f \in L^{r}(\Omega)$ with $Q r^{\prime}<Q_{1}$.

Then any renormalized solution of $(P V \lambda)$ is in $W_{0}^{1, p}(\Omega) \cap L^{\infty}(\Omega)$. Thus $\lambda_{b}=\lambda^{*}=\lambda_{r}$.

Remark 5.13 It holds in particular when $p=N, g$ satisfies (1.3) for some $Q \geqq N-1$ and $f \in L^{r}(\Omega), r>1$.

\subsection{Superlinear case: Extremal solutions}

In this paragraph we assume for simplicity that $g$ is defined on $[0, \infty)$.

Definition 5.14 Assume that $0<\lambda_{b} \leqq \lambda^{*} \leqq \lambda_{r}<\infty$. The function

$$
v^{*}=\sup _{\lambda \nearrow \lambda_{b}} \underline{v}_{\lambda}
$$

where $\underline{v}_{\lambda}$ is the minimal bounded solution of $(P V \lambda)$ is called extremal.

Remark 5.15 Assume that $g$ is at least linear near $\infty: \underline{\lim }_{\tau \longrightarrow \infty} g(\tau) / \tau>0$ (it holds in particular when $g$ is convex, $g \neq 0$ ).

(i) Then $\lambda_{r}<\infty$. Indeed there exists $c>0$ such that $1+g(\tau) \geqq c(1+\tau)$ for any $\tau \in[0, \infty)$. If $(P V \lambda)$ has a solution, then there exists a solution of problem

$$
-\Delta_{p} v=\lambda c^{1 /(p-1)} f(x)(1+v)^{p-1} \quad \text { in } \Omega, \quad w=0 \quad \text { on } \partial \Omega .
$$

Then $\lambda \leqq c^{-1 /(p-1)} \lambda_{1}(f)$ from Theorem 4.1 .

(ii) The function $v^{*}$ is well defined with values in $[0, \infty]$ as soon as $\mathcal{G}(f)<\infty$. For simplification, we will assume in the main results that $f \in L^{r}(\Omega), r>N / p$.

Next we study the case $g$ superlinear near $\infty$ :

$$
g \in C^{1}([0, \infty)), g(0)=0 \text { and } g \text { is nondecreasing, and } \lim _{s \longrightarrow \infty} \frac{g(s)}{s}=\infty .
$$

Here the first question is to know if $v^{*}$ satisfies the limit problem $\left(\mathrm{PV} \lambda_{b}\right)$ and in what sense.

The case $p=2$ was studied in [15] for $g$ convex, with $f=1$. In fact the proof does uses the convexity, and extends to more general $f$ and we recall it below. 
Lemma 5.16 ([15]) Assume $p=2$ and (5.10), $f \in L^{r}(\Omega), r>N / 2$. Then $v^{*}$ is a very weak solution of $\left(P V \lambda_{b}\right)$, that means $v^{*} \in L^{1}(\Omega), g\left(v^{*}\right) \in L^{1}(\Omega, \rho d x)$ where $\rho$ is the distance to $\partial \Omega$, and

$$
-\int_{\Omega} v^{*} \Delta \zeta d x=\int_{\Omega} f g\left(v^{*}\right) \zeta d x, \quad \forall \zeta \in C^{2}(\bar{\Omega}), \zeta=0 \text { on } \partial \Omega .
$$

Proof. Let $\lambda_{n} \nearrow \lambda_{b}$ and $v_{n}=\underline{v}_{\lambda_{n}}$; multiplying the equation relative to $v_{n}$ by a first eigenfunction $\Phi_{1}>0$ of the Laplacian with the weight $f$, one finds

$$
\lambda_{1}(f) \int_{\Omega} f v_{n} \Phi_{1} d x=\lambda_{n} \int_{\Omega} f\left(1+g\left(v_{n}\right)\right) \Phi_{1} d x
$$

and the superlinearity of $g$ implies that $\int_{\Omega} f\left(1+g\left(v_{n}\right)\right) \Phi_{1} d x$ is bounded, thus $\left(f g\left(v_{n}\right)\right)$ is bounded in $L^{1}(\Omega, \rho d x)$. Using the test function $\varphi=\mathcal{G}(1)$, it implies that $\left(v_{n}\right)$ is bounded in $L^{1}(\Omega)$ from the Höpf Lemma. Then $v^{*} \in L^{1}(\Omega)$ and satisfies (5.11).

When moreover $g$ is convex, it was proved in [54] that $v^{*}$ is more regular, in particular $g\left(v^{*}\right) \in$ $L^{1}(\Omega)$, by using stability properties of $v^{*}$. Thus $v^{*}$ is a renormalized solution of $\left(\mathrm{PV} \lambda^{*}\right)$. In case $p \neq 2$ there is no notion of very weak solution.

\subsubsection{Without convexity}

Without convexity we obtain a local result:

Proposition 5.17 Assume (5.10) and $f \in L^{r}(\Omega), r>N / p$. Then $v^{*}$ is a local renormalized solution of $\left(P V \lambda_{b}\right)$. In particular $T_{k}\left(v^{*}\right) \in W_{\text {loc }}^{1, p}(\Omega)$ for any $k>0, v^{* p-1} \in L_{\text {loc }}^{\sigma}(\Omega)$, for any $\sigma \in[1, N /(N-p))$, and $\left(\left|\nabla v^{*}\right|\right)^{p-1} \in L_{l o c}^{\tau}(\Omega)$, for any $\tau \in[1, N /(N-1))$, and

$$
-\Delta_{p} v^{*}=\lambda^{*} f\left(1+g\left(v^{*}\right)\right)^{p-1} \quad \text { in } \mathcal{D}^{\prime}(\Omega)
$$

For the proof we use the following Lemma:

Lemma 5.18 Assume $f \in L^{1}(\Omega)$, and $g$ satisfies (5.10). Let $\left(\lambda_{n}\right)$ be a sequence of reals such that $\underline{\lim } \lambda_{n}>0$, and $\left(v_{n}\right)$ be a sequence of renormalized solutions of problem $\left(P V \lambda_{n}\right)$. Then $\left(f g\left(v_{n}\right)^{p-1}\right)$ is bounded in $L_{l o c}^{1}(\Omega)$, and $\left(v_{n}^{p-1}\right)$ is bounded in $L_{l o c}^{\sigma}(\Omega)$, for any $\sigma \in[1, N /(N-p))$.

Proof of Lemma 5.18. From Lemma 2.16, for any $x_{0}$ such that $B\left(x_{0}, 4 \rho\right) \subset \Omega$, there exists a constant $C=C(N, p)$ such that

$$
\lambda_{n} \int_{B\left(x_{0}, \rho\right)} f\left(1+g\left(v_{n}\right)\right)^{p-1} d x \leqq C \rho^{N-p} \min _{B\left(x_{0}, \rho\right)} v_{n}^{p-1} \leqq \frac{C \rho^{N-p}}{\int_{B\left(x_{0}, \rho\right)} f d x} \int_{B\left(x_{0}, \rho\right)} f v_{n}^{p-1} d x
$$


Then there exist $c=c\left(N, p, \rho, f, x_{0}, \underline{\lim } \lambda_{n}\right)>0$ such that

$$
\int_{B\left(x_{0}, \rho\right)} f g\left(v_{n}\right)^{p-1} d x \leqq c \int_{B\left(x_{0}, \rho\right)} f v_{n}^{p-1} d x .
$$

From (5.10), there exists $A>0$ such that $g(t) \geqq(2 c)^{1 /(p-1)} t$ for any $t \geqq A$, thus

$$
\int_{B\left(x_{0}, \rho\right)} f g\left(v_{n}\right)^{p-1} d x \leqq c \int_{B\left(x_{0}, \rho\right)} f v_{n}^{p-1} d x \leqq 2 A^{p-1} c \int_{B\left(x_{0}, \rho\right)} f d x \leqq 2 A^{p-1} c\|f\|_{L^{1}(\Omega)},
$$

and the claim is proved. Moreover we deduce that

$$
\min _{B\left(x_{0}, \rho\right)} v_{n}^{p-1} \leqq c^{\prime}=c^{\prime}\left(N, p, \rho, f, g, x_{0}\right)
$$

from the weak Harnack inequality, $\left(v_{n}^{p-1}\right)$ is bounded in $L_{l o c}^{\sigma}(\Omega)$, for any $\sigma \in[1, N /(N-p))$.

Proof of Proposition 5.17, Let $\lambda_{n} \nearrow \lambda_{b}$, and $v_{n}=\underline{v}_{\lambda_{n}}$. From Lemma [5.18, $\left(f g\left(v_{n}\right)^{p-1}\right)$ is bounded in $L_{l o c}^{1}(\Omega)$, and $\left(v_{n}^{p-1}\right)$ is bounded in $L_{l o c}^{\sigma}(\Omega)$, for any $\sigma \in[1, N /(N-p))$. Then from [9, Theorem 3.2], there exists a subsequence converging a.e. in $\Omega$. And $\left(v_{n}\right)$ is nondecreasing thus the whole sequence converges to $v^{*}$. And $g$ is nondecreasing, thus $f g\left(v^{*}\right)^{p-1} \in L_{l o c}^{1}(\Omega)$ from the Beppo-Levi Theorem, and $\left(f g\left(v_{n}\right)^{p-1}\right)$ converges to $f g\left(v^{*}\right)^{p-1}$ weakly in $L_{l o c}^{1}(\Omega)$; thus $\left(\lambda_{n} f(x)(1+\right.$ $\left.g\left(v_{n}\right)\right)^{p-1}$ ) converges to $\lambda^{*} f(x)\left(1+g\left(v^{*}\right)\right)^{p-1}$ weakly in $L_{l o c}^{1}(\Omega)$. From [9, Theorem 3.3], $v^{*}$ is a local renormalized solution of $\left(\mathrm{PV} \lambda_{b}\right)$.

Our next results use the Euler function linked to the problem. From the Maximum Principle, problem $(\mathrm{PV} \lambda)$ is equivalent to

$$
-\Delta_{p} v=\lambda f(x) \varphi(v)=\lambda f(x)\left(1+g\left(v^{+}\right)\right)^{p-1} \quad \text { in } \Omega, \quad v=0 \quad \text { on } \partial \Omega .
$$

where

$$
\varphi(t)=\left(1+g\left(t^{+}\right)\right)^{p-1} ; \quad \Phi(t)=\int_{0}^{t} \varphi(s) d s=\int_{0}^{t}\left(1+g\left(s^{+}\right)\right)^{p-1} d s,
$$

thus $\Phi \in C^{1}(\mathbb{R})$. For any $f \in L^{1}(\Omega)$ and any $v \in W_{0}^{1, p}(\Omega)$ such that $f \Phi(v) \in L^{1}(\Omega)$, we set

$$
J_{\lambda}(v)=\frac{1}{p} \int_{\Omega}|\nabla v|^{p} d x-\lambda \int_{\Omega} f \Phi(v) d x .
$$

In particular the function $J_{\lambda}$ is defined on $W_{0}^{1, p}(\Omega) \cap L^{\infty}(\Omega)$. Let us recall some important properties of $J_{\lambda}$.

Proposition 5.19 ([19]) Assume $f \in L^{1}(\Omega)$ and (1.2). Let $\lambda>0$ such that there exists a supersolution $\bar{v} \in W_{0}^{1, p}(\Omega)$ of $(P V \lambda)$. Then $J_{\lambda}$ is defined on $\mathcal{K}_{\bar{v}}=\left\{v \in W_{0}^{1, p}(\Omega): 0 \leqq v \leqq \bar{v}\right\}$ and attains its minimum on $\mathcal{K}_{\bar{v}}$ at some point $v$ which is a solution of $(P V \lambda)$. In particular if $0<\lambda<\lambda_{b}$, then

$$
J_{\lambda}\left(\underline{v}_{\lambda}\right)=\min _{\mathcal{K}_{\underline{v}_{\lambda}}} J_{\lambda}(v) \leqq 0 .
$$


Remark 5.20 In fact $J_{\lambda}\left(\underline{v}_{\lambda}\right)<0$. Indeed if $J_{\lambda}\left(\underline{v}_{\lambda}\right)=0$, then for any $t \in(0,1), J_{\lambda}\left(\underline{t}_{\lambda}\right) \geqq 0$, thus

$$
t^{p} \int_{\Omega}\left|\nabla \underline{v}_{\lambda}\right|^{p} d x \geqq p \lambda \int_{\Omega} f \Phi\left(t \underline{v}_{\lambda}\right) d x \geqq p \lambda t \int_{\Omega} f \underline{\underline{v}}_{\lambda} d x
$$

thus $f \underline{v}_{\lambda}=0$, and $f>0$, thus $\underline{v}_{\lambda}=0$, which is contradictory.

Next we give a global result under the well-known Ambrosetti-Rabinowitz condition on $g$ :

Proposition 5.21 Assume (5.10), $f \in L^{r}(\Omega), r>N / p$ and

$$
\varliminf_{t \rightarrow \infty} t \varphi(t) / \Phi(t)=k>p .
$$

Then $v^{*} \in W_{0}^{1, p}(\Omega)$ and is a variational solution of $\left(P V \lambda_{b}\right)$.

Proof. Let $\lambda_{n} \nearrow \lambda_{b}$, and $v_{n}=\underline{v}_{\lambda_{n}}$. From Proposition $\underline{5.19}$,

$$
J_{\lambda_{n}}\left(v_{n}\right)=\frac{1}{p} \int_{\Omega}\left|\nabla v_{n}\right|^{p} d x-\lambda_{n} \int_{\Omega} f \Phi\left(v_{n}\right) d x \leqq 0
$$

and

$$
\int_{\Omega}\left|\nabla v_{n}\right|^{p} d x=\lambda_{n} \int_{\Omega} f\left(1+g\left(v_{n}\right)\right)^{p-1} v_{n} d x
$$

then there exists $B>0$ and $C>0$ such that

$$
\left.0 \geqq p J_{\lambda_{n}}\left(v_{n}\right)=\lambda_{n} \int_{\Omega} f\left(v_{n} \varphi\left(v_{n}\right)-p \Phi\left(v_{n}\right)\right) d x \geqq \frac{1}{2} \lambda_{n}(k-p) \int_{\left\{v_{n} \geqq B\right\}} f \Phi\left(v_{n}\right)\right) d x-C \lambda_{n}
$$

thus $f \Phi\left(v_{n}\right)$ is bounded in $L^{1}(\Omega)$, and also $\int_{\Omega}\left|\nabla v_{n}\right|^{p} d x$ is bounded; then there exists a subsequence converging weakly in $W_{0}^{1, p}(\Omega)$, and necessarily to $v^{*}$. From Proposition 5.17, $v^{*}$ is a solution of $\left(\mathrm{PV} \lambda_{b}\right)$ in $\mathcal{D}^{\prime}(\Omega)$, thus in the variational sense.

Remark 5.22 Proposition applies in particular when $\underline{\lim }_{t \rightarrow \infty} t g^{\prime}(t) / g(t)=m>1$. It follows from the L'Hospital rule, since $\left.(t \varphi(t))^{\prime} / \Phi^{\prime}(t)=1+(p-1) t g^{\prime}(t) /(1+g(t))\right)$ for any $t>0$. This improves the result of [19], where moreover it is supposed that $g(t) \leqq C\left(1+t^{m}\right)$, and extends also the one of [16].

\subsubsection{With convexity}

Here we assume that $g$ satisfies is superlinear and convex near $\infty$. Recall that $\lambda_{b}=\lambda^{*}=\lambda_{r}<\infty$ from Theorem 5.8 and Remark 5.15. We first define some functions linked to $g$ and give their asymptotic properties. 
Lemma 5.23 Assume (5.10) with $g$ convex near $\infty$. Let for any $t \geqq 0$

$$
\begin{gathered}
j(t)=t g^{\prime}(t)-g(t), \quad \mathcal{J}(t)=t \varphi(t)-p \Phi(t) \\
h(t)=\int_{0}^{t} g^{\prime}(s)\left(g^{\prime}(t)-g^{\prime}(s)\right) d s=g(t) g^{\prime}(t)-\int_{0}^{t} g^{\prime 2}(s) d s .
\end{gathered}
$$

Then $\lim _{t \rightarrow \infty} j(t) / g^{\prime}(t)=\infty, \lim _{t \rightarrow \infty} \mathcal{J}(t) / \varphi(t)=\infty$. and $\lim _{t \rightarrow \infty} h(t) / j(t)=\infty$.

Proof. (i) The function $j$ is nondecreasing near $\infty$, since $g$ is convex near $\infty$. Thus $j$ has a limit $L$ in $(-\infty, \infty]$. Let us show that $L=\infty$; indeed if $L$ is finite, then $t g^{\prime}(t) \leqq g(t)+|L|+1$ for large $t$, thus $(g(t)+|L|+1) / t$ is nonincreasing, which contradicts (5.10). First assume that $g \in C^{2}((0, \infty))$ and $g^{\prime \prime}(t)>0$ : from the l'Hospital rule,

$$
\lim _{t \rightarrow \infty} j(t) / g^{\prime}(t)=\lim _{t \rightarrow \infty} j^{\prime}(t) / g^{\prime \prime}(t)=\lim _{t \rightarrow \infty} t=\infty .
$$

In the general case $g$ is convex for $t \geqq A \geqq 0$, and $\lim _{t \rightarrow \infty} g^{\prime}(t)=\infty$; thus for any $K>0$, there exists $t_{K}>A+2 K$ such that $g^{\prime}(t) \geqq 2 g^{\prime}(A+2 K)$ for $t \geqq t_{K}$. Then for $t \geqq t_{K}$,

$$
\begin{aligned}
j(t) & =\int_{0}^{t}\left(g^{\prime}(t)-g^{\prime}(s)\right) d s=\int_{0}^{A}\left(g^{\prime}(t)-g^{\prime}(s)\right) d s+\int_{A}^{t}\left(g^{\prime}(t)-g^{\prime}(s)\right) d s \\
& \geqq-g(A)+\int_{A}^{A+2 K}\left(g^{\prime}(t)-g^{\prime}(s)\right) d s \geqq-g(a)+K g^{\prime}(t),
\end{aligned}
$$

thus $\lim _{t \rightarrow \infty} j(t) / g^{\prime}(t)=\infty$. And

$$
\mathcal{J}^{\prime}(t)=(p-1)(1+g(t))^{p-2}(j(t)-1)=\varphi^{\prime}(t)(j(t)-1) / g^{\prime}(t)
$$

thus $\lim _{t \rightarrow \infty} \mathcal{J}^{\prime}(t) / \varphi^{\prime}(t)=\infty$; and $\lim _{t \longrightarrow \infty} \varphi(t)=\infty$, thus $\lim _{t \rightarrow \infty} \mathcal{J}(t) / \varphi(t)=\infty$.

(ii) First assume that $g \in C^{2}((0, \infty))$ and $g^{\prime \prime}(t)>0$. Then $h(t)=\int_{0}^{t} g(s) g^{\prime \prime}(s) d s$, and from the l'Hospital rule,

$$
\lim _{t \rightarrow \infty} h(t) / j(t)=\lim _{t \rightarrow \infty} h^{\prime}(t) / t g^{\prime \prime}(t)=\lim _{t \rightarrow \infty} g(t) / t=\infty .
$$

In the general case, for any $C>g^{\prime}(A)$, there exists $A_{1}>A>0$ such that $g^{\prime}(s) \geqq 2 C$, for $s \geqq A_{1}$ and $g^{\prime}(s) \leqq 2 C$ for $s \leqq A_{1}$ and there exists $B>5 A_{1}$ such that $g^{\prime}(t) \geqq 2 g^{\prime}\left(5 A_{1}\right)$ for $t \geqq B$. Then denoting $C_{A}=h(A)-C j(A)$, for $t \geqq B$,

$$
\begin{aligned}
h(t)-C j(t) & =C_{A}+\int_{A}^{t}\left(g^{\prime}(s)-C\right)\left(g^{\prime}(t)-g^{\prime}(s)\right) d s \\
& \geqq-\left|C_{A}\right|-C A_{1}\left(g^{\prime}(t)+2 C\right)+\int_{A_{1}}^{5 A_{1}}\left(g^{\prime}(s)-C\right)\left(g^{\prime}(t)-g^{\prime}(s)\right) d s \\
& \geqq-\left|C_{A}\right|-C A_{1}\left(g^{\prime}(t)+C\right)+2 A_{1} C g^{\prime}(t)=-\left|C_{A}\right|+C A_{1}\left(g^{\prime}(t)-C\right)
\end{aligned}
$$


thus $\lim _{t \rightarrow \infty} h(t) / j(t)=\infty$.

The following result will be used also in next Paragraph. The proof is new, using only the function $\mathcal{J}$. Notice that the proof given in [2] for $p=2$ was not extendable.

Proposition 5.24 Assume (5.10) with $g$ convex near $\infty$, and $f \in L^{1}(\Omega)$. Let $\left(\lambda_{n}\right)$ be a sequence of positive reals such that $\underline{\lim } \lambda_{n}>0$, and $\left(v_{n}\right)$ be a sequence of solutions of $\left(P V \lambda_{n}\right)$, such that $v_{n} \in W_{0}^{1, p}(\Omega), f \Phi\left(v_{n}\right) \in L^{1}(\Omega)$, and $J_{\lambda_{n}}\left(v_{n}\right) \leqq c \in \mathbb{R}$.

Then $\left(\Delta_{p} v_{n}\right)$ is bounded in $L^{1}(\Omega)$.

Proof. The function $v_{n} \in W_{0}^{1, p}(\Omega)$ still satisfies (5.16), thus

$$
p J_{\lambda_{n}}\left(v_{n}\right)=\lambda_{n} \int_{\Omega} f\left(v_{n} \varphi\left(v_{n}\right)-p \Phi\left(v_{n}\right)\right) d x=\lambda_{n} \int_{\Omega} f \mathcal{J}\left(v_{n}\right) d x \leqq c p,
$$

where $\mathcal{J}$ is defined at (5.17). Then from Lemma 5.23, $\int_{\Omega} f \varphi\left(v_{n}\right) d x$ is bounded, which means that $\left(\Delta_{p} v_{n}\right)$ is bounded in $L^{1}(\Omega)$.

As a consequence, we prove that the extremal solution is a solution of $\left(\mathrm{PV} \lambda^{*}\right)$ in a very simple way:

Corollary 5.25 Assume (5.10) with $g$ convex near $\infty$, and $f \in L^{r}(\Omega)$ with $r>N / p$. Then the extremal solution $v^{*}$ is a renormalized solution of $\left(P V \lambda^{*}\right)$.

Proof. Let $\lambda_{n} \nearrow \lambda^{*}$, and $v_{n}=\underline{v}_{\lambda_{n}}$. Then $J_{\lambda_{n}}\left(v_{n}\right) \leqq 0$ from Proposition 5.19, From Proposition 5.24. $\left(f g\left(v_{n}\right)^{p-1}\right)$ is bounded in $L^{1}(\Omega)$, and $\left(v_{n}^{p-1}\right)$ is bounded in $L^{\sigma}(\Omega)$, for any $\sigma \in[1, N /(N-p))$. Then from [9, Theorem 3.2], converges to $v^{*}$ a.e. in $\Omega$, as in Proposition 5.17. From the BeppoLevi theorem, $f g\left(v^{*}\right)^{p-1} \in L^{1}(\Omega)$, and $\left(f g\left(v_{n}\right)^{p-1}\right)$ converges to $f g(v)^{p-1}$ weakly in $L^{1}(\Omega)$; thus $\left(\lambda_{n} f(x)\left(1+g\left(v_{n}\right)\right)^{p-1}\right)$ converges to $\lambda^{*} f(x)(1+g(v))^{p-1}$ weakly in $L^{1}(\Omega)$. From Remark 2.11, $v$ is a renormalized solution of $\left(\mathrm{PV} \lambda^{*}\right)$.

Next we find again this result and get further informations on $v^{*}$ by using stability properties of the minimal bounded solutions. This extend the results of [54] for $p=2$ and of [64] for $p>2$ with $f \equiv 1$. Here we use the function $h$ defined at (5.18), introduced by [54]. We first extend the definition given in [19] for functions $v \in W_{0}^{1, p}(\Omega)$ :

Definition 5.26 A renormalized solution $v$ of problem $(P V \lambda)$ is called semi-stable if the "second derivative of $J_{\lambda}$ is nonnegative", in the sense

$$
\int_{\{\nabla v \neq 0\}}|\nabla v|^{p-2}\left((p-2)\left(\frac{\nabla v \cdot \nabla \psi}{|\nabla v|}\right)^{2}+|\nabla \psi|^{2}\right) d x \geqq(p-1) \lambda \int_{\Omega} f(1+g(v))^{p-2} g^{\prime}(v) \psi^{2} d x,
$$

for any $\psi \in \mathcal{D}(\Omega)$ if $p \geqq 2 ;$ for any $\psi \in \mathcal{D}(\Omega)$ such that $\psi \leqq C v$ and $|\nabla \psi| \leqq C|\nabla v|$ in $\Omega$ for some $C>0$ if $p<2$. 
The integral on the left-hand side is well defined. Indeed either $p>2$ and $|\nabla v|^{p-1} \in L^{1}(\Omega)$, or $p<2$ and

$$
\int_{\{\nabla v \neq 0\}}|\nabla v|^{p-2}|\nabla \psi|^{2} d x \leqq C \int_{\{|\nabla v|>1\}}|\nabla v|^{p-1}|\nabla \psi| d x+\int_{\{0<|\nabla v| \leqq 1\}}|\nabla \psi|^{2} d x
$$

When $v \in W_{0}^{1, p}(\Omega)$, (5.21) is valid for any $\psi \in W_{0}^{1, p}(\Omega)$, satisfying the conditions above when $p<2$.

Proposition 5.27 Assume (5.10) with $g$ convex near $\infty$, and $f \in L^{r}(\Omega), r>N / p$. Let $h$ be defined at (5.18). (i) Then

$$
f\left(1+g\left(v^{*}\right)\right)^{p-1} h\left(v^{*}\right) \in L^{1}(\Omega) .
$$

(ii) If $N<N_{0}=p p^{\prime} /(1+1 /(p-1) r)$, then $v^{*} \in L^{\infty}(\Omega)$.

If $N>N_{0}$, then $v^{* p-1} \in L^{k}(\Omega)$ for any $k<\bar{\sigma}$, where $1 / \bar{\sigma}=1-p p^{\prime} / N+1 / r(p-1)$.

If $N=N_{0}$, then $v^{*} \in L^{k}(\Omega)$ for any $k \geqq 1$.

(iii) If $N<N_{1}=p\left(1+p^{\prime}\right) /\left(1+p^{\prime} / r\right)$ then $v^{*} \in W_{0}^{1, p}(\Omega)$.

If $N>N_{1},\left|\nabla v^{*}\right|^{p-1} \in L^{\tau}(\Omega)$ for any $\tau<\bar{\tau}$ where $1 / \bar{\tau}=1+1 /(p-1) r-\left(p^{\prime}+1\right) / N$.

If $N=N_{1},\left|\nabla v^{*}\right| \in L^{s}(\Omega)$ for any $s<p$.

(iv) If $\underline{\lim }_{t \rightarrow \infty} h(t) / t>0$, then $v^{*} \in W_{0}^{1, p}(\Omega)$. It holds in particular if $\underline{\lim }_{t \rightarrow \infty}\left(g^{\prime}(t)-g(t) / t\right)>0$.

Proof. (i) Let $\lambda_{n} \nearrow \lambda^{*}$, and $v_{n}=\underline{v}_{\lambda_{n}}$. By hypothesis $g$ is convex for $t \geqq A$. From [19, Proposition 2.2], $v_{n}$ is semi-stable. Taking $\psi=g\left(v_{n}\right)$ in (5.21) with $\lambda=\lambda_{n}$ and $v=v_{n}$, we get

$$
\int_{\Omega}\left|\nabla v_{n}\right|^{p} g^{\prime 2}\left(v_{n}\right) d x \geqq \lambda_{n} \int_{\Omega} f\left(1+g\left(v_{n}\right)\right)^{p-2} g^{\prime}\left(v_{n}\right) g^{2}\left(v_{n}\right) d x .
$$

Taking $S\left(v_{n}\right)$,as a test function in $\left(\mathrm{PV} \lambda_{n}\right)$, where $S(t)=\int_{0}^{t} g^{\prime 2}(s) d s$, we find

$$
\int_{\Omega}\left|\nabla v_{n}\right|^{p} g^{\prime 2}\left(v_{n}\right) d x=\lambda_{n} \int_{\Omega} f\left(1+g\left(v_{n}\right)\right)^{p-1} S\left(v_{n}\right) d x
$$

By difference we obtain

$$
\begin{aligned}
& \int_{\Omega} f\left(1+g\left(v_{n}\right)\right)^{p-2}\left(\left(1+g\left(v_{n}\right)\right) S\left(v_{n}\right)-g^{\prime}\left(v_{n}\right) g^{2}\left(v_{n}\right)\right) d x \\
& =\int_{\Omega} f\left(1+g\left(v_{n}\right)\right)^{p-2}\left(S\left(v_{n}\right)-g\left(v_{n}\right) h\left(v_{n}\right)\right) d x \geqq 0 .
\end{aligned}
$$

Observing that $S(t) \leqq g(t) g^{\prime}(t)+|h(A)|$ for $t \geqq A$, and $\lim _{t \rightarrow \infty} h(t) / g^{\prime}(t)=\infty$, from Lemma 5.23, there exists $C>0$ such that

$$
\left.\int_{\Omega} f\left(1+g\left(v_{n}\right)\right)^{p-2} g\left(v_{n}\right) h\left(v_{n}\right)\right) d x \leqq C .
$$


And $\lim _{t \rightarrow \infty} g(t)=\infty$, thus $1+g(t) \leqq 2 g(t)$ for $t \geqq A$, hence $\left(f\left(1+g\left(v_{n}\right)\right)^{p-1} h\left(v_{n}\right)\right)$ is bounded in $L^{1}(\Omega)$, thus (5.22) holds. Then $f g\left(v^{*}\right)^{p-1} j\left(v^{*}\right) \in L^{1}(\Omega)$ from Lemma 5.23, hence $f g\left(v^{*}\right)^{p-1} g^{\prime}\left(v^{*}\right) \in$ $L^{1}(\Omega)$ and $f g\left(v^{*}\right)^{p} / v^{*} \in L^{1}(\Omega)$. In particular we find again that $\left(f\left(1+g\left(v^{*}\right)\right)^{p-1)} \in L^{1}(\Omega)\right.$, which was obtained in a shorter way at Theorem 5.25 .

(ii)The regularity of $v^{*}$ follows from the estimate $f\left(g\left(v^{*}\right)\right)^{p} / v^{*} \in L^{1}(\Omega)$ : Taking $r^{\prime}<\sigma<N /(N-p)$, we have $v^{* p-1} \in L^{\sigma}(\Omega)$. Defining $\theta$ by $p / \theta=p-1+1 / r+1 / \sigma$, we have $\theta \in\left(1, p^{\prime}\right)$, and from Hölder inequality,

$$
\begin{aligned}
\int_{\Omega}\left(f g\left(v^{*}\right)^{p-1}\right)^{\theta} d x & =\int_{\Omega}\left(\frac{f^{1 / p} g\left(v^{*}\right)}{v^{* 1 / p}}\right)^{(p-1) \theta}\left(f^{\theta / p} v^{* \theta / p^{\prime}}\right) d x \\
& \leqq\left(\int_{\Omega} \frac{f g\left(v^{*}\right)^{p}}{v^{*}} d x\right)^{\theta / p^{\prime}}\left(\int_{\Omega}\left(f^{\theta / p} v^{* \theta / p^{\prime}}\right)^{p^{\prime} /\left(p^{\prime}-\theta\right)} d x\right)^{1-\theta / p^{\prime}} \\
& \leqq\left(\int_{\Omega} \frac{f g\left(v^{*}\right)^{p}}{v^{*}} d x\right)^{\theta / p^{\prime}}\left(\int_{\Omega} f^{r} d x\right)^{\theta / p}\left(\int_{\Omega} v^{* \sigma(p-1)} d x\right)^{\theta / p \sigma}
\end{aligned}
$$

Then $f g\left(v^{*}\right)^{p-1} \in L^{\theta}(\Omega)$ with $\theta>1$. If $p=N$, then from Lemma 2.13, $v^{*} \in L^{\infty}(\Omega)$. Next assume $p<N$. Choosing $\sigma$ sufficiently close to $r^{\prime}$, one has $\theta<N / p$. From Lemma 2.13, as soon as $\theta<N / p$, we find $v^{* p-1} \in L^{\sigma_{1}}(\Omega)$ with $\sigma_{1}=N \theta /(N-p \theta)$. For $\sigma$ sufficiently close to $r^{\prime}$, we also find $\sigma_{1}>\sigma$. Then we can define an increasing sequence $\left(\sigma_{\nu}\right)$ and a sequence $\left(\theta_{\nu}\right)$, as long as $\theta_{\nu}<N / p$. If $\left(\sigma_{\nu}\right)$ has a limit $\bar{\sigma}$, then $1 / \bar{\sigma}=1-p p^{\prime} / N+1 / r(p-1)$, and $\left(\theta_{\nu}\right)$ converges to $\bar{\theta}=\left(1+1 / r(p-1)-p^{\prime} / N\right)^{-1}$. It follows that $v^{*} \in L^{\infty}(\Omega)$ if $N<N_{0}$. If $N \geqq N_{0}, v^{* p-1} \in L^{k}(\Omega)$ for any $k<\bar{\sigma}$.

(iii) Lemma 2.13 also gives estimates of the gradient: if $p=N$, then $v^{*} \in W_{0}^{1, N}(\Omega)$. If $p<N$, $\left(\left|\nabla v^{*}\right|^{p-1}\right) \in L^{\tau_{\nu}}(\Omega)$ with $1 / \tau_{\nu}=1 / \theta_{\nu}-1 / N$, as long as $\theta_{\nu}<N p /(N p-N+p)$, and $\left(\tau_{\nu}\right)$ converges to $\bar{\tau}=\left(1+1 /(p-1) r-\left(p^{\prime}+1\right) / N\right)^{-1}$. Then $v^{*} \in W_{0}^{1, p}(\Omega)$ if $\bar{\tau}>p^{\prime}$, that means if $N<N_{1}$. If $N \geqq N_{1},\left(\left|\nabla v^{*}\right|^{p-1}\right) \in L^{\tau}(\Omega)$ for any $\tau<\bar{\tau}$.

(iv) If $\underline{\lim }_{t \rightarrow \infty} h(t) / t>0$, then

$$
\int_{\Omega}\left|\nabla v_{n}\right|^{p} d x=\lambda_{n} \int_{\Omega} f\left(1+g\left(v_{n}\right)\right)^{p-1} v_{n} d x \leqq C
$$

thus $v^{*} \in W_{0}^{1, p}(\Omega)$. It holds in particular when $\underline{\lim }_{t \rightarrow \infty} j(t) / t>0$, from Lemma 5.23 .

Remark 5.28 if $p \geqq 2, v^{*}$ is semi-stable. Indeed $v_{n}=\underline{v}_{\lambda_{n}}$ satisfies (5.21) for any $\psi \in \mathcal{D}(\Omega)$. And $\left(\left|\nabla v_{n}\right|\right)$ converges strongly in $L^{1}(\Omega)$ to $\left|\nabla v^{*}\right|^{p-1}$, so that we can go to the limit from Lebesgue Theorem and Fatou Lemma.

Remark 5.29 In case $p=2, \Omega$ strictly convex, and $f=1$, then $v^{*} \in W_{0}^{1,2}(\Omega)$, for any function $g$ satisfying (5.10), from [55]. The proof uses the fact that $J_{\lambda^{*}}\left(v^{*}\right) \leqq 0$ and Pohozaev identity; the kea point is that $v^{*}$ is regular near the boundary, from results of [62]. In the general case $p>1$ with 
$f \equiv 1$, if we can prove that $v^{*}$ is regular near the boundary, then $v^{*} \in W_{0}^{1, p}(\Omega)$. Indeed Pohozaev identity extends to the p-Laplacian, see [40]. For general $f$ we cannot get the result by this way, even for $p=2$.

Remark 5.30 In the exponential case $1+g(v)=e^{v}$, with $f \equiv 1$, it has been proved that $v^{*} \in$ $W_{0}^{1, p}(\Omega)$, and $v^{*} \in L^{\infty}(\Omega)$ whenever $N<N_{2}=4 p /(p-1)+p$, see [32] and [35]. In the power case, $(1+g(v))^{p-1}=(1+v)^{m}$ the same happens; if $N \geqq N_{2}$, and $m<m_{c}$, where

$$
m_{c}=\frac{(p-1) N-2 \sqrt{(p-1)(N-1)}+2-p}{N-p-2-2 \sqrt{(N-1) /(p-1)}}
$$

then also $v^{*} \in L^{\infty}(\Omega)$, see [28]. The same conclusions hold when the function $g$ behaves like an exponential or a power, see [67], [19], [63], and [27]. Up to our knowledge, the gap between $N_{0}=p p^{\prime}$ and $\mathrm{N}_{2}$ remains for general $g$, excepted in the radial case, see [18].

We end this paragraph with a boundness property when $g$ has a slow growth:

Proposition 5.31 Assume that $g$ satisfies (5.10) and (1.3) for some $Q \in\left(p-1, Q_{1}\right)$, and $g$ is convex near $\infty$, and $f \in L^{r}(\Omega)$ with $Q r^{\prime}<Q_{1}$.

Then $v^{*} \in W_{0}^{1, p}(\Omega) \cap L^{\infty}(\Omega)$ and is a variational solution of $\left(P V \lambda^{*}\right)$.

Proof. As in Proposition 5.12, it follows from Corollary [5.25] and Proposition 2.14 (i).

\subsection{Boundedness and multiplicity under Sobolev conditions}

Next we assume only that $g$ is subcritical with the Sobolev exponent:

$$
\varlimsup_{\tau \longrightarrow \infty} \frac{g(\tau)^{p-1}}{\tau^{Q}}<\infty, \text { for some } Q \in\left(p-1, Q^{*}\right)
$$

and $f \in L^{r}(\Omega)$ with $(Q+1) r^{\prime}<p^{*}$. Then $J_{\lambda}$ is well defined on $W_{0}^{1, p}(\Omega)$ and $J_{\lambda} \in C^{1}\left(W_{0}^{1, p}(\Omega)\right)$.

Proposition 5.32 Assume (5.10) and (5.23), $g$ convex near $\infty$, and $f \in L^{r}(\Omega)$ with $(Q+1) r^{\prime}<p^{*}$. Let $\left(\lambda_{n}\right)$ be a sequence of positive reals such that $\lim \lambda_{n}=\lambda>0$, and $\left(v_{n}\right)$ be a sequence of solutions of $\left(P V \lambda_{n}\right)$ such that $v_{n} \in W_{0}^{1, p}(\Omega)$, and $J_{\lambda_{n}}\left(v_{n}\right) \leqq c \in \mathbb{R}$.

Then $\left(v_{n}\right)$ is bounded in $W_{0}^{1, p}(\Omega)$.

Proof. We still have

$$
p J_{\lambda_{n}}\left(v_{n}\right)=\lambda_{n} \int_{\Omega} f\left(v_{n} \varphi\left(v_{n}\right)-p \Phi\left(v_{n}\right)\right) d x=\lambda_{n} \int_{\Omega} f \mathcal{J}\left(v_{n}\right) d x
$$


where $\mathcal{J}$ is defined at (5.17). From Proposition 5.24, $\left(f g\left(v_{n}\right)^{p-1}\right)$ is bounded in $L^{1}(\Omega)$. Following the method of $([43])$, suppose that up to a subsequence, $\lim \left\|v_{n}\right\|_{W_{0}^{1, p}(\Omega)}=\infty$, and consider $w_{n}=$ $v_{n} /\left\|v_{n}\right\|_{W_{0}^{1, p}(\Omega)}$. Up to a subsequence, $\left(w_{n}\right)$ converges to a function $w$ weakly in $W_{0}^{1, p}(\Omega)$ and strongly in $L^{k+1}(\Omega)$, for any $k<Q^{*}$. For any $\zeta \in \mathcal{D}(\Omega)$,

$$
\int_{\Omega}\left|\nabla w_{n}\right|^{p-2} \nabla w_{n} \nabla \zeta d x=\left\|v_{n}\right\|_{W_{0}^{1, p}(\Omega)}^{1-p} \int_{\Omega}\left(1+g\left(v_{n}\right)\right)^{p-1} \zeta d x
$$

tends to 0 , thus $w=0$. Let $z_{n}=t_{n} v_{n}$, where

$$
t_{n}=\inf \left\{t \in[0,1]: J_{\lambda_{n}}\left(t v_{n}\right)=\max _{s \in[0,1]} J_{\lambda_{n}}\left(s v_{n}\right)\right\} .
$$

In fact $\lim J_{\lambda_{n}}\left(z_{n}\right)=\infty$. Indeed suppose that $\underline{\lim } J_{\lambda_{n}}\left(z_{n}\right)=M<\infty$. For given $K>0$, setting $u_{n}=$ $K w_{n}$, then up to a subsequence, $J_{\lambda_{n}}\left(u_{n}\right) \leqq J_{\lambda_{n}}\left(z_{n}\right) \leqq M+1$ for large $n$. And $\lim \int_{\Omega} f \Phi\left(u_{n}\right) d x=0$, from (5.23) and the assumptions on $f$, hence $\lim J_{\lambda_{n}}\left(u_{n}\right)=\lambda K^{p} / p$ from (5.14). Taking $K$ large enough leads to a contradiction. Then $t_{n} \in(0,1)$ for large $n$, thus

$$
\begin{gathered}
J_{\lambda_{n}}^{\prime}\left(z_{n}\right)\left(z_{n}\right)=\int_{\Omega}\left|\nabla z_{n}\right|^{p} d x-\lambda_{n} \int_{\Omega} f\left(1+g\left(z_{n}\right)\right)^{p-1} z_{n} d x=0, \\
\lambda_{n}^{-1} p J_{\lambda_{n}}\left(z_{n}\right)=\int_{\Omega} f\left(z_{n} \varphi\left(z_{n}\right)-p \Phi\left(z_{n}\right)\right) d x=\int_{\Omega} f \mathcal{J}\left(z_{n}\right) d x .
\end{gathered}
$$

And $\lim _{t \rightarrow \infty} j(t)=\infty$, from Lemma 5.23. Thus there exists $B>0$ such that $j(s)-1>0$ for $s \geqq B$, hence $\mathcal{J}(B) \leqq \mathcal{J}(t) \leqq \mathcal{J}(\tau)$ for any $B \leqq t \leqq \tau$ from (5.19). Moreover $z_{n} \leqq v_{n}$ a.e. in $\Omega$, thus $\left\{z_{n}>B\right\} \subset\left\{v_{n}>B\right\}$, then with different constants $C>0$,

$$
\int_{\Omega} f \mathcal{J}\left(z_{n}\right) d x \leqq C+\int_{\left\{z_{n}>B\right\}} f \mathcal{J}\left(z_{n}\right) d x \leqq C+\int_{\left\{v_{n}>B\right\}} f \mathcal{J}\left(v_{n}\right) d x \leqq C+\int_{\Omega} f \mathcal{J}\left(v_{n}\right) d x \leqq C+\lambda_{n}^{-1} p c
$$

therefore $\left(J_{\lambda_{n}}\left(z_{n}\right)\right)$ is bounded, and we reach a contradiction. Then $\left(v_{n}\right)$ is bounded in $W_{0}^{1, p}(\Omega)$.

As a consequence we obtain the boundedness of the extremal solution under estimate [5.23, which achieves the proof of Theorem [1.4.

Proposition 5.33 Assume (5.10) and (5.23), $g$ convex near $\infty$, and $f \in L^{r}(\Omega)$ with $(Q+1) r^{\prime}<p^{*}$. Then the extremal solution $v^{*} \in W_{0}^{1, p}(\Omega) \cap L^{\infty}(\Omega)$ and is a variational solution of $\left(P V \lambda^{*}\right)$.

Proof. Considering $\lambda_{n} \nearrow \lambda^{*}$, the sequence of minimal solutions $v_{n}=\underline{v}_{\lambda_{n}}$ satisfies $J_{\lambda_{n}}\left(v_{n}\right) \leqq 0$ from Proposition 5.19. From Proposition [5.32, $\left(v_{n}\right)$ is bounded in $W_{0}^{1, p}(\Omega)$, and converges to $v^{*}$ a.e. in $\Omega$, thus $v^{*} \in W_{0}^{1, p}(\Omega)$ and is a variational solution of $\left(\mathrm{PV} \lambda^{*}\right)$. Then $v \in L^{\infty}(\Omega)$ from Proposition 2.14 (iii). 
Next we show the multiplicity result of Theorem [1.5, where $f, g$ satisfy the assumptions of Proposition 5.32, We still use the Euler function $J_{\lambda}$ associated to (PV $\lambda$ ). Here two difficulties occur. For small $\lambda, J_{\lambda}$ has the geometry of Mountain Path near 0, but function $g$ can have a slow growth, and one cannot prove that the Palais-Smale sequences are bounded in $W_{0}^{1, p}(\Omega)$; then we use a result of [42] saying that there exist $\left(\lambda_{n}\right)$ converging to $\lambda$, such that $J_{\lambda_{n}}$ has a critical point $v_{n}$, and we prove that this sequence $\left(v_{n}\right)$ is bounded. For larger $\lambda$ it is not sure that $J_{\lambda}$ has the geometry of Mountain Path near the minimal solution $\underline{v}_{\lambda}$ of $(\operatorname{PV} \lambda)$, and we have to make further assumptions on $g$.

Proof of Theorem 1.5. For any $\lambda \in\left(0, \lambda^{*}\right)$ there exists at least one solution, the minimal one $\underline{v}_{\lambda}$, such that $J_{\lambda}\left(\underline{v}_{\lambda}\right)<0$, from Proposition 5.19 and Remark 5.20 ,

\section{(i) Existence of a second solution for $\lambda$ small enough.}

From (5.10) and (5.23), there exists $\lambda_{0} \in\left(0, \lambda^{*}\right)$ such that for any $\lambda<\lambda_{0}$, there exists $R_{\lambda}>0$ such that $\inf \left\{J_{\lambda}(v):\|v\|_{W_{0}^{1, p}(\Omega)}=R_{\lambda}\right\}>0$, and a function $w_{\lambda} \in W_{0}^{1, p}(\Omega)$ with $\left\|w_{\lambda}\right\|_{W_{0}^{1, p}(\Omega)}>R_{\lambda}$ and $J_{\lambda}\left(w_{\lambda}\right)<0$. Then $J_{\lambda}$ has the geometry of the Mountain Path near 0:

$$
c_{\lambda}=\inf _{\theta \in \Gamma} \max _{t \in[0,1]} J_{\lambda}(\theta(t))>0=\max \left(J_{\lambda}(0), J_{\lambda}\left(w_{\lambda}\right)\right),
$$

where $\Gamma=\left\{\theta \in C\left([0,1], W_{0}^{1, p}(\Omega)\right): \theta(0)=0, \theta(1)=w_{\lambda}\right\}$. Let $\lambda_{1} \in\left(0, \lambda_{0}\right)$ be fixed. Let us show the existence of a solution at the level $c_{\lambda_{1}}$. There exists $\delta>0$ such that the family of functions $\left(J_{\lambda}\right)_{\alpha \in\left[\lambda_{1}(1-\delta), \lambda_{1}(1+\delta)\right]}$ also satisfy the condition (5.24):

$$
c_{\lambda}=\inf _{\theta \in \Gamma} \max _{t \in[0,1]} J_{\lambda}(\theta(t))>0=\max \left(J_{\lambda}(0), J_{\lambda}\left(w_{\lambda_{1}}\right)\right) .
$$

From [42, for almost every $\lambda \in\left[\lambda_{1}(1-\delta), \lambda_{1}(1+\delta]\right.$, there exists a sequence $\left(v_{\lambda, m}\right)$, bounded in $W_{0}^{1, p}(\Omega)$, such that $\lim J_{\lambda}\left(v_{\lambda, m}\right)=c_{\lambda}$ and $\lim J_{\lambda}^{\prime}\left(v_{\lambda, m}\right)=0$ in $W^{-1, p^{\prime}}(\Omega)$. From (5.23) , the PalaisSmale condition holds: there exists a subsequence, converging to a function $v_{\lambda}$ strongly in $W_{0}^{1, p}(\Omega)$, and $J_{\lambda}\left(v_{\lambda}\right)=c_{\lambda}$, and $J_{\lambda}^{\prime}\left(v_{\lambda}\right)=0$, in other words $v_{\lambda}$ is a solution of $(\mathrm{PV} \lambda)$. This holds for a sequence $\left(\lambda_{n}\right)$ converging to $\lambda_{1}$. Let $v_{n}=v_{\lambda_{n}}$, then $v_{n}$ is a solution of $\left(\mathrm{PV} \lambda_{n}\right)$, thus

$$
J_{\lambda_{n}}\left(v_{n}\right)=\lambda_{n} \int_{\Omega} f\left(v_{n} \varphi\left(v_{n}\right)-p \Phi\left(v_{n}\right)\right) d x=c_{\lambda_{n}} \leqq c_{\lambda}+1 .
$$

From Proposition [5.32, $\left(v_{n}\right)$ is also bounded in $W_{0}^{1, p}(\Omega)$. Up to a subsequence $\left(v_{n}\right)$ converges to a function $v$ weakly in $W_{0}^{1, p}(\Omega)$ and strongly in $L^{k}(\Omega)$ for any $k<p^{*}$, and a.e. in $\Omega$. Then $\left(\lambda_{n} f\left(1+g\left(v_{n}\right)\right)^{p-1}\right)$ converges to $\lambda_{1} f(1+g(v))^{p-1}$ strongly in $L^{1}(\Omega)$. From Remark 2.11, $v$ is a solution of $\left(\mathrm{PV} \lambda_{1}\right)$. And $\left(f\left(v_{n} \varphi\left(v_{n}\right)-p \Phi\left(v_{n}\right)\right)\right)$ converges to $f(v \varphi(v)-p \Phi(v))$ strongly in $L^{1}(\Omega)$ then $\left(J_{\lambda_{n}}\left(v_{n}\right)\right)=\left(c_{\lambda_{n}}\right)$ converges to $J_{\lambda}(v)$, thus $J_{\lambda}(v)=c_{\lambda}$.

(i) Existence of a second solution for $\lambda<\lambda^{*}$. 
Let $\lambda_{1}<\lambda^{*}$ be fixed. Let $\lambda_{2} \in\left(\lambda_{1}, \lambda^{*}\right)$, and let $\underline{v}_{\lambda_{1}}, \underline{v}_{\lambda_{2}}$ be the minimal bounded solutions associated to $\lambda_{1}, \lambda_{2}$. Then on $\left[0, \underline{v}_{\lambda_{2}}\right]$ there exists a solution $v_{0}$ minimizing $J_{\lambda_{1}}$. From Proposition 5.19, $v_{0}$ is a solution of $\left(\mathrm{PV} \lambda_{1}\right)$ and $\underline{v}_{\lambda_{1}}$ is minimal, thus $\underline{v}_{\lambda_{1}} \leqq v_{0} \leqq \underline{v}_{\lambda_{2}}$.

- First suppose $p=2$ and $g$ is convex. Then $v_{0}=\underline{v}_{\lambda_{1}}$ and it is a strict minimum of $J_{\lambda_{1}}$. Indeed $\underline{v}_{\lambda_{2}}$ is semi-stable, thus for any $\varphi \in W_{0}^{1,2}(\Omega)$,

$$
\int_{\Omega}|\nabla \varphi|^{2} d x \geqq \lambda_{2} \int_{\Omega} f g^{\prime}\left(\underline{v}_{\lambda_{2}}\right) \varphi^{2} d x
$$

and $g^{\prime}\left(\underline{v}_{\lambda_{2}}\right) \geqq g^{\prime}\left(\underline{v}_{\lambda_{1}}\right)$, thus

$$
J_{\lambda_{1}}^{\prime \prime}\left(\underline{v}_{\lambda_{1}}\right) \cdot(\varphi, \varphi)=\int_{\Omega}|\nabla \varphi|^{2} d x-\lambda_{1} \int_{\Omega} f g^{\prime}\left(\underline{v}_{\lambda_{1}}\right) \varphi^{2} d x \geqq\left(1-\frac{\lambda_{1}}{\lambda_{2}}\right) \int_{\Omega}|\nabla \varphi|^{2} d x ;
$$

and $J_{\lambda_{1}}^{\prime}\left(\underline{v}_{\lambda_{1}}\right)=0$, then $\underline{v}_{\lambda_{1}}$ is a strict local minimum in $W_{0}^{1, p}(\Omega)$. Then there exists $R_{\lambda_{1}}>0$ and $w_{\lambda_{1}} \in W_{0}^{1, p}(\Omega)$ with $\left\|w_{\lambda 1}\right\|_{W_{0}^{1, p}(\Omega)}>R_{\lambda_{1}}$ such that

$$
\inf \left\{J_{\lambda}(v):\left\|v-\underline{v}_{\lambda_{1}}\right\|_{W_{0}^{1, p}(\Omega)}=R_{\lambda_{1}}\right\}>J_{\lambda_{1}}\left(\underline{v}_{\lambda_{1}}\right)>J_{\lambda_{1}}\left(w_{\lambda_{1}}\right) .
$$

Therefore $J_{\lambda_{1}}$ has the geometry of the Mountain Path near $\underline{v}_{\lambda_{1}}$. Using the results of [42] as above, we get the existence of a solution of $\left(\mathrm{PV}_{\lambda_{1}}\right)$ at a level $c_{\lambda_{1}}>J_{\lambda_{1}}\left(\underline{v}_{\lambda_{1}}\right)$, different from $\underline{v}_{\lambda_{1}}$.

- Next suppose that $g$ satisfies condition (5.15), without convexity assumption, and $f \in L^{\infty}(\Omega)$. If $v_{0} \neq \underline{v}_{\lambda_{1}}$ we have constructed a second solution. Next assume that $v_{0}=\underline{v}_{\lambda_{1}}$. Since $f \in L^{\infty}(\Omega)$, $\underline{v}_{\lambda_{2}}$ and $v_{0} \in C^{1, \alpha}(\bar{\Omega})$. From [34, Theorem 5.2], $v_{0}$ is a local minimum in $W_{0}^{1, p}(\Omega)$ : it minimizes $J_{\lambda_{1}}$ in a ball $B\left(v_{0}, \delta\right)$ of $W_{0}^{1, p}(\Omega)$. From (5.15), we get $t \varphi(t) \geqq(k+p) \Phi(t) / 2$ for $t>A>0$. Here the Palais-Smale sequences are bounded: if $v_{n} \in W_{0}^{1, p}(\Omega)$ satisfies $\lim J_{\lambda_{1}} .\left(v_{n}\right)=c$ and if $\xi_{n}=J_{\lambda_{1}}^{\prime}\left(v_{n}\right)$ tends to 0 in $W^{-1, p^{\prime}}(\Omega)$, one finds, with different constants $C>0$,

$$
\begin{aligned}
\int_{\Omega}\left|\nabla v_{n}\right|^{p} d x-\xi_{n}\left(v_{n}\right) & =\lambda_{1} \int_{\Omega} f v_{n}^{+} \varphi\left(v_{n}^{+}\right) d x-\int_{\Omega} f v_{n}^{-} d x \geqq \lambda_{1} \int_{\left\{v_{n} \geqq A\right\}} f v_{n}^{+} \varphi\left(v_{n}^{+}\right) d x-C\left\|v_{n}\right\|_{W_{0}^{1, p}(\Omega)} \\
& \geqq \lambda_{1} \frac{k+p}{2} \int_{\left\{v_{n} \geqq A\right\}} f \Phi\left(v_{n}\right) d x-C\left\|v_{n}\right\|_{W_{0}^{1, p}(\Omega)} \\
& \geqq \frac{k+p}{2 p} \int_{\Omega}\left|\nabla v_{n}\right|^{p} d x-C\left(1+\left\|v_{n}\right\|_{W_{0}^{1, p}(\Omega)}\right)
\end{aligned}
$$

thus $\left(v_{n}\right)$ is bounded in $W_{0}^{1, p}(\Omega)$. And there exists a function $\tilde{v}$ such that $J_{\lambda_{1}} .(\tilde{v})<J_{\lambda_{1}}$. $\left(v_{1}\right)$ and $\left\|\underline{v}_{\lambda_{1}}-\tilde{v}\right\| \geqq 1+\delta$. Let

$$
\tilde{c}_{\lambda}=\inf _{\theta \in \Gamma} \max _{t \in[0,1]} J_{\lambda}(\theta(t)) \geqq \max \left(J_{\lambda_{1}}\left(\underline{v}_{\lambda_{1}}\right), J_{\lambda_{1}}(\tilde{v})\right)
$$


where $\Gamma=\left\{\theta \in C\left([0,1], W_{0}^{1, p}(\Omega)\right): \theta(0)=\underline{v}_{\lambda_{1}}, \theta(1)=w_{\lambda}\right\}$. And $\underline{v}_{\lambda_{1}}$ is a local minimum. Then either the inequality is strict and there exists a solution at level $\tilde{c}_{\lambda}$ from Moutain Path Theorem. Or $\tilde{c}_{\lambda}=J_{\lambda_{1}}\left(v_{1}\right)$ and there exists a solution in $W_{0}^{1, p}(\Omega) \backslash B\left(\underline{v}_{\lambda_{1}}, \delta\right)$, from the variant of [37].

Remark 5.34 In case $p=N$, assumptions of growth are not needed in Propositions 5.31 and 5.33: for any $g$ satisfying (5.10), convex near $\infty$, and $f \in L^{r}(\Omega), r>1$, we have $v^{*} \in W_{0}^{1, N}(\Omega) \cap L^{\infty}(\Omega)$, from Proposition 5.27. However assumption (1.3) for some $Q>N-1$ is required in order to get the multiplicity result of Theorem 1.5 .

\section{Problem $(\mathrm{PV} \lambda)$ with measures}

Here we study the existence of a renormalized solution of problem

$$
-\Delta_{p} v=\lambda f(1+g(v))^{p-1}+\mu \quad \text { in } \Omega, \quad v=0 \quad \text { on } \partial \Omega
$$

where $\mu \in \mathcal{M}_{b}^{+}(\Omega), \mu \neq 0$. The problem is not easy for $p \neq 2$. Indeed the convergence and stability results relative to problem (2.2) are still restrictive, see Theorem 2.6.

Remark 6.1 In order to obtain an existence result, an assumption of slow growth condition is natural, as well as more assuptions on $f$. Take for example $p=2<N$ and $g(v)=v^{Q}$ for some $Q>0$, and let $\mu=\delta_{a}$ be a Dirac mass at some point $a \in \Omega$. If $v$ is a solution, then $v(x) \geqq C|x-a|^{2-N}$ near a; then necessarily $|x-a|^{(2-N) Q} f \in L^{1}(\Omega)$; then $Q<N /(N-2)$ if $f \equiv 1$. More generally if there exists a solution of (6.1), then $f \mathcal{G}(\mu) \in L^{1}(\Omega)$, where $\mathcal{G}(\mu)$ is the potential of $\mu$. This condition is always satisfied if $f \in L^{r}(\Omega)$ for some $r>N / 2$.

The existence result of Theorem [1.6 is a consequence of the next theorem, where $\mu \in \mathcal{M}_{b}(\Omega)$ is arbitrary, without assumption of sign. It improves a result announced in [39, Theorem 1.1.] for $Q>1$, with an incomplete proof. Our result covers the general case $Q>0$, and gives better informations in the case $Q=p-1$. We give here a detailed proof, valid for any $p \leqq N$, where the approximation of the measure is precised.

Theorem 6.2 Consider the problem

$$
-\Delta_{p} U=\lambda h(x, U)+\mu \quad \text { in } \Omega, \quad U=0 \quad \text { on } \partial \Omega,
$$

where $\mu \in \mathcal{M}_{b}(\Omega)$, and

$$
|h(x, U)| \leqq f(x)\left(K+|U|^{Q}\right),
$$

with $Q>0$ and $\lambda, K>0$, and $f \in L^{r}(\Omega)$ with $Q r^{\prime}<Q_{1}$. Then there exists a renormalized solution of (6.2) in any of the following cases:

$$
\begin{aligned}
& Q=p-1 \quad \text { and } \quad \lambda<\lambda_{1}(f) ; \\
& 0<Q<p-1 ; \\
& Q>p-1 \quad \text { and } \quad \lambda\|f\|_{L^{r}(\Omega)}\left(\lambda K\|f\|_{L^{r}(\Omega)}+|\mu|(\Omega)\right)^{(Q-p+1) /(p-1)}|\Omega|^{1 / r^{\prime}-Q / Q_{1}} \leqq C
\end{aligned}
$$


for some $C=C(N, p, Q)$ for $p<N$, and $C=C\left(N, Q, K_{N}(\Omega)\right)$ for $p=N$.

Proof. (i) Construction of a suitable approximation of $\mu$. Let

$$
\mu=\mu_{1}-\mu_{2}+\mu_{s}^{+}-\mu_{s}^{-}, \quad \text { with } \mu_{1}=\mu_{0}^{+}, \mu_{2}=\mu_{0}^{-} \in \mathcal{M}_{0}^{+}(\Omega), \quad \mu_{s}^{+}, \mu_{s}^{-} \in \mathcal{M}_{s}^{+}(\Omega),
$$

thus $\mu_{1}(\Omega)+\mu_{2}(\Omega)+\mu_{s}^{+}(\Omega)+\mu_{s}^{-}(\Omega) \leqq 2|\mu(\Omega)|$. Following the proof of [12], see also [26], for $i=1,2$, one has

$$
\mu_{i}=\varphi_{i} \gamma_{i}, \quad \text { with } \gamma_{i} \in \mathcal{M}_{b}^{+}(\Omega) \cap W^{-1, p^{\prime}}(\Omega) \text { and } \varphi_{i} \in L^{1}\left(\Omega, \gamma_{i}\right) .
$$

Let $\left(K_{n}\right)_{n \geqq 1}$ a increasing sequence of compacts of union $\Omega$, and set $\nu_{1, i}=T_{1}\left(\varphi_{i} \chi_{K_{1}}\right) \gamma_{i}$ and $\nu_{n, i}=$ $T_{n}\left(\varphi_{i} \chi_{K_{n}}\right) \gamma_{i}-T_{n-1}\left(\varphi_{i} \chi_{K_{n-1}}\right) \gamma_{i}$. By regularization there exist nonnegative $\phi_{n, i} \in \mathcal{D}(\Omega)$ such that $\left\|\phi_{n, i}-\nu_{n, i}\right\|_{W^{-1, p^{\prime}}(\Omega)} \leqq 2^{-n} \mu_{i}(\Omega)$. Then $h_{n, i}=\sum_{1}^{n} \phi_{k, i} \in \mathcal{D}(\Omega)$ and $\left(h_{n, i}\right)$ converges strongly in $L^{1}(\Omega)$ to a function $h_{i}$, and $\left\|h_{n, i}\right\|_{L^{1}(\Omega)} \leqq \mu_{i}(\Omega)$. Also $G_{n, i}=\sum_{1}^{n}\left(\nu_{k, i}-\phi_{k, i}\right) \in W^{-1, p^{\prime}}(\Omega) \cap \mathcal{M}_{b}(\Omega)$ and $\left(G_{n, i}\right)$ converges strongly in $W^{-1, p^{\prime}}(\Omega)$ to some $G_{i}$, and $\mu_{i}=h_{i}+G_{i}$, and $\left\|G_{n, i}\right\|_{\mathcal{M}_{b}(\Omega)} \leqq 2 \mu_{i}(\Omega)$. Otherwise by regularization there exist nonnegative $\lambda_{n}^{1}$ and $\lambda_{n}^{2} \in \mathcal{D}(\Omega)$ converging respectively to $\mu_{s}^{+}, \mu_{s}^{-}$in the narrow topology, with $\left\|\lambda_{n}^{1}\right\|_{L^{1}(\Omega)} \leqq \mu_{s}^{+}(\Omega),\left\|\lambda_{n}^{2}\right\|_{L^{1}(\Omega)} \leqq \mu_{s}^{-}(\Omega)$. Then the sequence of approximations of $\mu$ defined by

$$
\mu_{n}=h_{n, 1}-h_{n, 2}+G_{n, 1}-G_{n, 2}+\lambda_{n}^{1}-\lambda_{n}^{2}
$$

satisfies the conditions of stability of Theorem 2.6, and moreover is bounded with respect to $|\mu|(\Omega)$ by a universal constant:

$$
\left|\mu_{n}\right|(\Omega) \leqq 4|\mu|(\Omega)
$$

(ii) The approximate problem. For any fixed $n \in \mathbb{N}$, we search a variational solution of

$$
-\Delta_{p} U_{n}=\lambda T_{n}\left(h\left(x, U_{n}\right)\right)+\mu_{n}
$$

by using the Schauder Theorem. To any $V \in W_{0}^{1, p}(\Omega)$ we associate the solution $U=\mathcal{F}_{n}(V) \in$ $W_{0}^{1, p}(\Omega)$ of

$$
-\Delta_{p} U=\lambda T_{n}(h(x, V))+\mu_{n},
$$

where $T_{n}$ is the truncation function. We find $\|\nabla U\|_{L^{p}(\Omega)}^{p} \leqq \lambda n\|U\|_{L^{1}(\Omega)}+\left\|\mu_{n}\right\|_{W^{-1, p^{\prime}}(\Omega)}\|U\|_{W_{0}^{1, p}(\Omega)}$, thus $\|U\|_{W_{0}^{1, p}(\Omega)} \leqq C_{n}$ independent on $V$. Let $B_{n}=B\left(0, C_{n}\right)$ be the ball of $W_{0}^{1, p}(\Omega)$ of radius $C_{n}$. Then $\mathcal{F}_{n}$ is continuous and compact from $B_{n}$ into itself, thus it has a fixed point $U_{n}$. From Proposition 2.2 and Remark 2.3, using (2.6) with $\sigma=Q r^{\prime} /(p-1)$, we have

$$
\begin{aligned}
\left(\int_{\Omega}\left|U_{n}\right|^{Q r^{\prime}} d x\right)^{(p-1) / Q r^{\prime}} & \leqq C_{0}|\Omega|^{\ell}\left(\lambda \int_{\Omega}\left|T_{n}\left(h\left(x, U_{n}\right)\right)\right| d x+\left|\mu_{n}(\Omega)\right|\right) \\
& \left.\leqq C_{0}|\Omega|^{\ell}\left(\lambda\|f\|_{L^{r}(\Omega)} \int_{\Omega}\left|U_{n}\right|^{Q r^{\prime}} d x\right)^{1 / r^{\prime}}+\lambda K\|f\|_{L^{r}(\Omega)}+4|\mu|(\Omega)\right),
\end{aligned}
$$


with $\ell=(p-1) / Q r^{\prime}-(N-p) / N$, and $C_{0}=C_{0}(N, p, Q, r)$ for $p<N$, and $C_{0}=C_{0}\left(N, Q, r, K_{N}(\Omega)\right)$ for $p=N$.

(iii) Case $Q<p-1$. Then from (6.7), $\left(\left|U_{n}\right|^{Q r^{\prime}}\right)$ is bounded in $L^{1}(\Omega)$. In turn $\left(h\left(x, U_{n}\right)\right)$ is bounded in $L^{1}(\Omega)$, thus $\left(-\Delta_{p} U_{n}\right)$ is bounded in $L^{1}(\Omega)$. Then $\left(\left|U_{n}\right|^{p-1}\right)$ is bounded in $L^{s}(\Omega)$ for any $s \in[1, N /(N-p)$ ) (any $s \geqq 1$ if $p=N)$. Choosing $s>Q r^{\prime} /(p-1)$, it follows that $\left(\left|h\left(x, U_{n}\right)\right|\right)$ is bounded in $L^{1+\varepsilon}(\Omega)$ for some $\varepsilon>0$. From Theorem 2.6 we can extract a subsequence converging a.e. in $\Omega$ to a renormalized solution of problem (6.2).

(iv) Case $Q=p-1$. Assume $\lambda<\lambda_{1}(f)$. Let us show again that $\left(\left|U_{n}\right|^{Q r^{\prime}}\right)$ is bounded in $L^{1}(\Omega)$. If not, up to a subsequence, $a_{n}=\int_{\Omega}\left|U_{n}\right|^{(p-1) r^{\prime}} d x$ tends to $\infty$ and we set $w_{n}=a_{n}^{-1 /(p-1) r^{\prime}} U_{n}$. Then $w_{n} \in W_{0}^{1, p}(\Omega), \int_{\Omega} w_{n}^{(p-1) r^{\prime}} d x=1$, and satisfies

$$
-\Delta_{p} w_{n}=\eta_{n}+\varphi_{n}, \quad \eta_{n}=a_{n}^{-1 / r^{\prime}} \lambda T_{n}\left(h\left(x, U_{n}\right)\right), \quad \varphi_{n}=a_{n}^{-1 / r^{\prime}} \mu_{n} .
$$

And $\left(\varphi_{n}\right)$ converges to 0 strongly in $L^{1}(\Omega)$, and $\left(\eta_{n}\right)$ is bounded in $L^{1}(\Omega)$, since $f \in L^{r}(\Omega)$ and

$$
\left|\eta_{n}\right| \leqq \psi_{n}=\lambda f\left(C a_{n}^{-1 / r^{\prime}}+\left|w_{n}\right|^{p-1}\right) .
$$

From [25, Section 5.1], up to a subsequence, $\left(w_{n}\right)$ converges a.e. in $\Omega$ to a function $w$. And $\left(w_{n}^{(p-1) s}\right)$ is bounded in $L^{1}(\Omega)$, for any $s<N /(N-p)$, and $r^{\prime}<N /(N-p)$, thus $\left(\left|w_{n}\right|^{(p-1) r^{\prime}}\right)$ converges strongly in $L^{1}(\Omega)$ to $|w|^{(p-1) r^{\prime}}$; hence $w \not \equiv 0$. And $\left(\psi_{n}\right)$ converges strongly in $L^{1}(\Omega)$ to $\lambda f|w|^{p-1}$, hence $\left(\eta_{n}\right)$ converges strongly to some $\eta \in L^{1}(\Omega)$. Therefore, $w$ is a renormalized solution of problem

$$
-\Delta_{p} w=\eta, \quad \text { in } \Omega, \quad \text { and }|\eta| \leqq \lambda f(x)|w|^{p-1} \text { a.e. in } \Omega .
$$

From Proposition 2.14 (i), we get $w \in W_{0}^{1, p}(\Omega)$, since $r>N / p$. Then

$$
\lambda_{1}(f) \int_{\Omega}|w|^{p} d x \leqq \int_{\Omega}|\nabla w|^{p} d x \leqq \lambda \int_{\Omega} f|w|^{p} d x
$$

which is contradictory. Then as above there exists a renormalized solution of problem (6.2).

(v) Case $Q>p-1$. Here the estimate of $\left(\left|U_{n}\right|^{Q r^{\prime}}\right)$ does not hold, but we construct a special approximation $\left(U_{n}\right)$ satisfying the estimate: we still have, for any $V \in W_{0}^{1, p}(\Omega)$ and $U=\mathcal{F}_{n}(V)$,

$$
\left.\left(\int_{\Omega}|U|^{Q r^{\prime}} d x\right)^{(p-1) / Q r^{\prime}} \leqq C_{0}|\Omega|^{\ell}\left(\lambda\|f\|_{L^{r}(\Omega)} \int_{\Omega}|V|^{Q r^{\prime}} d x\right)^{1 / r^{\prime}}+\lambda K\|f\|_{L^{r}(\Omega)}+4|\mu|(\Omega)\right) .
$$

Setting

$$
\left.x(V)=\int_{\Omega}|V|^{Q r^{\prime}} d x\right)^{(p-1) / Q r^{\prime}}, \quad a=C_{0}|\Omega|^{\ell}\left(\lambda K\|f\|_{L^{r}(\Omega)}+4|\mu|(\Omega)\right), \quad b=C_{0}|\Omega|^{\ell} \lambda\|f\|_{L^{r}(\Omega)},
$$


we find $x(U) \leqq a+b x(V)^{Q /(p-1)}$. Since $Q>p-1$, then $x(U)<x(V)$ as soon as $a^{(Q-p+1) /(p-1)} b \leqq$ $C(p, Q)$, which is assumed in (6.5) and $x(V) \leqq y=y(a, b, p, Q)$ small enough. Using the Schauder Theorem in the set of functions $V \in W_{0}^{1, p}(\Omega)$ such that $x(V) \leqq y$ and $\|U\|_{W_{0}^{1, p}(\Omega)} \leqq C_{n}$ there exists a solution $U_{n} \in W_{0}^{1, p}(\Omega)$ of (6.6) such that $\int_{\Omega}\left|U_{n}\right|^{Q r^{\prime}} d x$ is bounded. We conclude as before.

Remark 6.3 In case $Q=p-1$, condition (6.3) is sharp, from Theorem 1.2. The proof given above for $Q>p-1$ still works for $Q=p-1$, but condition (6.5) obtained in that case is not sharp.

We end this paragraph by an non existence result.

Proposition 6.4 Let $\mu_{s} \in \mathcal{M}_{s}^{+}(\Omega)$ be any singular measure.

(i) For any $\lambda>\lambda_{1}(f)$, or $\lambda=\lambda_{1}(f)$ and $f \in L^{N / p}(\Omega), p<N$, there is no solution $v$ of

$$
-\Delta_{p} v=\lambda f(1+v)^{p-1}+\mu_{s} \quad \text { in } \Omega, \quad v=0 \quad \text { on } \partial \Omega .
$$

(ii) Let $g$ be defined on $[0, \infty)$ and $\underline{\lim }_{t \rightarrow \infty} g(\tau) / \tau>0$. If $\lambda>\lambda_{r}$, there is no solution $v$ of

$$
-\Delta_{p} v=\lambda f(1+g(v))^{p-1}+\mu_{s} \quad \text { in } \Omega, \quad v=0 \quad \text { on } \partial \Omega .
$$

Proof. It follows from Lemma 2.17; if there exists a solution with a measure, there exists a solution without measure. In case (i) it follows also from Theorems 4.1 and 1.1) problem (1.4) has no solution, thus the same happens for problem (6.10).

\section{Applications to problem (PU $\lambda)$}

From the existence results obtained for problem $(\mathrm{PV} \lambda)$, we deduce existence results for problem (PU $\lambda$ ) by using Theorem 1.1. Starting from a function $\beta$ satisfying (1.1), we associate to $\beta$ the function $g$ defined by the change of unknown, namely by (3.1). We recall that if $\beta$ is defined on $[0, \infty)$, then also is $g$; conversely if $g$ is defined on $[0, \infty)$, then $L<\infty$ if and only if $1 /(1+g(v)) \in$ $L^{1}((0, \infty))$, from Remark 3.1. In some results we assume that $g$ satisfies (1.3):

$$
\varlimsup_{\tau \longrightarrow \infty} \frac{g(\tau)^{p-1}}{\tau^{Q}}<\infty
$$

for some $Q>0$ or equivalently

$$
\varlimsup_{t \rightarrow L} \frac{e^{\gamma(t)}}{\Psi^{Q}(t)}<\infty .
$$

In the case $\beta$ constant Theorem 1.2 follows:

Proof of Theorem 1.2, Any renormalized solution $u$ of (1.4) satisfies $(p-1)|\nabla u|^{p} \in L^{1}(\Omega)$, thus $u \in W_{0}^{1, p}(\Omega)$.. If $\lambda<\lambda_{1}(f)$, there exists a unique solution $v_{0} \in W_{0}^{1, p}(\Omega)$ of (4.4) fromTheorem 
4.1. Then from Theorem 1.1, $u_{0}=H\left(v_{0}\right)$ is a solution of (1.4) such that $v_{0}=\Psi\left(u_{0}\right)=e^{u_{0}}-1 \in$ $W_{0}^{1, p}(\Omega)$. Reciprocally, if $u$ is a solution of (1.4), such that $v=\Psi(u) \in W_{0}^{1, p}(\Omega)$, then from Theorem 1.1, $v$ is a reachable solution of

$$
-\Delta_{p} v=\lambda f(1+v)^{p-1}+\mu
$$

for some measure $\mu \in \mathcal{M}_{b}^{+}(\Omega)$. Since $v \in W_{0}^{1, p}(\Omega)$, then $\mu \in \mathcal{M}_{0}(\Omega)$. Then from existence and uniqueness of the solutions of (2.2) when $\mu \in \mathcal{M}_{0}(\Omega), v$ is also a renormalized solution; as in the proof of Theorem 1.1 (case $p=2$ or $N$ ), it follows that $\mu \in M_{s}^{+}(\Omega)$, thus $\mu=0$, and $v=v_{0}$, then $u=u_{0}$. If $f \in L^{N / p}(\Omega)$, then $v_{0} \in L^{k}(\Omega)$ for any $k>1$, and also $u_{0}$, since $u_{0} \leqq v_{0}$. If $f \in L^{r}(\Omega), r>N / p$, then $u_{0}, v_{0} \in L^{\infty}(\Omega)$; and for any $\mu_{s} \in M_{s}^{+}(\Omega)$ there exists a solution $v_{s}$ of (1.17) from Theorem 1.6, thus a corresponding solution $u_{s} \in W_{0}^{1, p}(\Omega)$ of (1.4). The nonexistence follows from Proposition 6.4 .

Our next result follows from Corollary [5.9, Theorem 1.6 and Propositions [5.10, 5.12,

Corollary 7.1 Assume that $\beta$ satisfies (1.1) with $L=\infty$.

(i) Suppose that $g$ satisfies (1.3) with $Q=p-1$.

If $M_{p-1} \lambda<\lambda_{1}(f)$, there exists at least a solution $u \in W_{0}^{1, p}(\Omega)$ to problem (PUA). If moreover $f \in L^{N / p}(\Omega), p<N$, then $u \in L^{k}(\Omega)$ for any $k>1$. If $f \in L^{r}(\Omega), r>N / p$, then $u \in L^{\infty}(\Omega)$; and there exists an infinity of less regular solutions $u_{s} \in$ of $(P U \lambda)$.

(ii) Suppose (1.3) with $Q<p-1$, and $f \in L^{r}(\Omega)$ with $r \in(1, N / p)$ such that $Q r^{\prime}<Q_{1}$.

Then for any $\lambda>0$ there exists a renormalized solution $u$ of $(P V \lambda)$ such that $v=\Psi(u)$ satisfies $v^{d} \in L^{1}(\Omega)$ for $d=N r(p-1-Q) /(N-p r)$. If $(Q+1) r^{\prime} \leqq p^{*}$, then $u \in W_{0}^{1, p}(\Omega)$. If $(Q+1) r^{\prime}>p^{*}$, then $|\nabla u|^{\theta} \in L^{1}(\Omega)$ for $\theta=N r(p-1-Q) /(N-(Q+1) r)$. There exists also an infinity of less regular solutions $u_{s}$ of (PUX).

(iii) Suppose (1.3) with $p-1<Q<Q_{1}$, and $f \in L^{r}(\Omega)$ with $Q r^{\prime}<Q_{1}$.

Then for $\lambda>0$ small enough, there exists a solution $u \in W_{0}^{1, p}(\Omega) \cap L^{\infty}(\Omega)$ of $(P U \lambda)$, and an infinity of less regular solutions.

From Proposition 5.1 and Theorem 5.8 we deduce the following:

Corollary 7.2 (i) Assume (1.1), and $f \in L^{r}(\Omega), r>N / p$. Then for $\lambda>0$ small enough, there exists a minimal solution $\underline{u}_{\lambda} \in W_{0}^{1, p}(\Omega) \cap L^{\infty}(\Omega)$ of $(P U \lambda)$, with $\left\|\underline{u}_{\lambda}\right\|_{L^{\infty}(\Omega)}<L$.

(ii) Suppose moreover that $\underline{\lim }_{t \rightarrow L} \beta(t)>0$ and $t \beta(t)$ is nondecreasing near $L$, and $f \not \equiv 0$. Then there exists $\lambda^{*}>0$ such that $L$;

if $\lambda \in\left(0, \lambda^{*}\right)$ there exists a minimal solution $\underline{u}_{\lambda} \in W_{0}^{1, p}(\Omega) \cap L^{\infty}(\Omega)$ of $(P U \lambda)$, with $\left\|\underline{u}_{\lambda}\right\|_{L^{\infty}(\Omega)}<$ if $\lambda>\lambda^{*}$ there exists no renormalized solution.

From Theorems and 1.4, and 1.5 and Remark 3.2, we obtain the following: 
Corollary 7.3 Assume (1.1) and $f \in L^{r}(\Omega), r>N / p, f \not \equiv 0$. Suppose that $\beta$ is nondecreasing near $L$ and $\lim _{t \rightarrow L} \beta(t)=\infty$, and $e^{\gamma(t) /(p-1)} \notin L^{1}(\Omega)$.

(i) Then $u^{*}=\sup _{\lambda} \lambda^{*} \underline{u}_{\lambda}$ is a solution of $\left(P U \lambda^{*}\right)$, and $u^{*} \in W_{0}^{1, p}(\Omega)$. If one of the conditions (i) (ii) (iii) of Theorem 1.4 holds, then $\left\|u^{*}\right\|_{L^{\infty}(\Omega)}<L$.

(ii) Suppose moreover that (1.3) holds with $Q<Q^{*}$, and $f \in L^{r}(\Omega)$ with $(Q+1) r^{\prime}<p^{*}$. Then for small $\lambda>0$ there exists at least two solutions of $(P U \lambda)$ such that $\|u\|_{L^{\infty}(\Omega)}<L$. It is true for any $\lambda<\lambda^{*}$ when $p=2$ and $\beta$ is nondecreasing.

\subsection{Remarks on growth assumptions}

Condition (7.1) is not easy to verify. It is implied by

$$
\varlimsup_{t \longrightarrow L} \frac{\beta(t)}{\Psi^{Q /(p-1)-1}(t)}<\infty
$$

from the L'Hospital rule. If moreover $\beta$ is nondecreasing, the two conditions are equivalent.

Remark 7.4 If $\beta=\beta_{1}+\beta_{2}$, where $\beta_{1} \in L^{1}((0, L))$ and $\Lambda_{2}=\infty$ and $\beta_{2}$ satisfies (7.1), then $\beta$ satisfies (7.1). Indeed setting $v=\Psi(u), v_{1}=\Psi_{1}(u)$ and $v_{2}=\Psi_{2}(u)$, one finds $v_{2} \leqq v$ and

$$
\frac{1+g(v)}{v^{q}} \leqq e^{\gamma(L) /(p-1)} \frac{1+g_{2}\left(v_{2}\right)}{v^{q}} \leqq e^{\gamma(L) /(p-1)} \frac{1+g_{2}\left(v_{2}\right)}{v_{2}^{q}} .
$$

In particular (7.1) is satisfied with $Q=p-1$ by any $\beta$ of this form, such that $\beta_{2}$ is bounded.

Next we give a simple condition on $\beta$ ensuring (7.1):

Lemma 7.5 Let $Q>0$. Assume that $\beta \in C^{1}([0, L))$, and $L=\infty$ or only $e^{\gamma(\theta) /(p-1)} \notin L^{1}((0, L))$, and

$$
\varlimsup_{t \rightarrow L} \frac{\beta^{\prime}}{\beta^{2}}(t) \leqq 1-\frac{p-1}{Q} .
$$

Then (7.1) holds.

Proof. The conditions imply $\Lambda=\infty$ and

$$
\varlimsup_{t \rightarrow L} \frac{\beta^{\prime}}{\beta^{2}}(t)=\varlimsup_{t \rightarrow L} \frac{g g^{\prime \prime}}{g^{\prime 2}}(\Psi(t))=\varlimsup_{\tau \longrightarrow \infty} \frac{g g^{\prime \prime}}{g^{\prime 2}}(\tau)
$$

then (7.3) implies that $g^{(p-1) / Q}$ is concave near $\infty$, thus at most linear. 
Remark 7.6 As observed in [2], many "elementary" nondecreasing functions $\beta$ on $[0, \infty)$ satisfy condition (7.1) for any $Q>p-1$. In the examples of Section 3, we have seen that for $\beta(u)=u^{m}$, $m>0, g(v)=O\left(v(\ln v)^{m /(m+1)}\right)$ near $\infty$. For $\beta(u)=e^{u}, g(v)=O(v \ln v)$ near $\infty$. For $\beta(u)=$ $e^{e^{u}+u}+e^{u}+1, g(v)=O(v \ln v \ln (\ln v))$. In those cases, $\varlimsup_{t \rightarrow \infty}\left(\beta^{\prime} / \beta^{2}\right)(t)=0$.

An open question raised in [2] and also [23] was to know if any nondecreasing $\beta$ defined on $[0, \infty)$ satisfies (7.1) for some $Q>p-1$. Here we show that condition (7.1) is not always satisfied, even with large $Q$, even when $\tau^{Q}$ is replaced by an exponential:

Lemma 7.7 Consider any function $F \in C^{0}([0, \infty))$ strictly convex, with $\lim _{s \rightarrow \infty} F(s)=\infty$. Then there exists a function $\beta \in C^{0}\left([0, \infty)\right.$, increasing with $\beta(0) \geqq 0, \lim _{t \rightarrow \infty} \beta(t)=\infty$ such that the corresponding function g given by (1.8) satisfies

$$
\varlimsup_{\tau \longrightarrow \infty} \frac{g(\tau)}{F(\tau)}=\infty
$$

Proof. From Remark 3.1 there is a one-to-one mapping between such a function and a function $g \in C^{1}([0, \infty))$, convex, such that $\lim _{s \longrightarrow \infty} g(s) / s=\infty$, and

$$
1 /(1+g(s)) \notin L^{1}((0, \infty)) .
$$

Thus it is sufficient to show the existence of such a function $g$ satisfying (7.4). We first construct a function $g$ which is only continuous. Let $\mathcal{F}$ be the curve defined by $F$. Set $g(s)=0$ for $s \in[0,1]$. There exists $m_{1}>1$ such that the line of slope $m_{1}$ issued from $(1,0)$ cuts $\mathcal{F}$ at two points $s_{1}^{\prime}<s_{1}^{\prime \prime}$. Then we define $g(s)=m_{1}(s-1)$ for any $s \in\left[1, s_{1}\right]$, where $s_{1}>s_{1}^{\prime \prime}$ is chosen such that $s_{1}-1 \geqq$ $(1+g(1)) e^{m_{1}}$, that means $s_{1} \geqq 1+e^{m_{1}}$. Then

$$
\int_{1}^{s_{1}} d s /(1+g(s) \geqq 1
$$

and the point $\left(s_{1}, g\left(s_{1}\right)\right)$ is under $\mathcal{F}$. By induction for any $n \geqq 1$, we consider $m_{n}>2 m_{n-1}$ such that the line of slope $m_{n}$ issued from $\left(s_{n-1}, g\left(s_{n-1}\right)\right)$ cuts the curve $\mathcal{F}_{n}$ defined by $n F$ at two points $s_{n}^{\prime}<s_{n}^{\prime \prime}$. We define $g(s)=g\left(s_{n-1}\right)+m_{n}\left(s-s_{n-1}\right)$ for any $s \in\left[s_{n-1}, s_{n}\right]$, where $s_{n}>s_{n}^{\prime \prime}$ is chosed such that $s_{n}-s_{n-1} \geqq\left(1+g\left(s_{n-1}\right)\right) e^{m_{n}}$ and $s_{n} \geqq 2 s_{n-1}$. Then

$$
\int_{s_{n-1}}^{s_{n}} d s /(1+g(s) \geqq 1 \text {. }
$$

The function $g$ satisfies $1 /(1+g(s)) \notin L^{1}((0, \infty))$, and $g \geqq n F$ on $\left[s_{n}^{\prime}, s_{n}^{\prime \prime}\right]$, and $s_{n}^{\prime}>s_{n}>1$, thus (17.4) holds; and $g\left(s_{n}\right) \geqq m_{n}\left(s_{n}-s_{n-1}\right) \geqq m_{n} s_{n} / 2$, then $\lim _{s \longrightarrow \infty} g(s) / s=\infty$. Then we regularize $g$ near the points $s_{n}$ in order to get a $C^{1}$ convex function. 


\subsection{Extensions}

1) In the correlation Theorem [1.1, we can assume that $f$ depends also on $u$ or $v$. If $u$ is a solution of a problem of the form

$$
-\Delta_{p} u=\beta(u)|\nabla u|^{p}+\lambda f(x, u),
$$

where $f(x, u) \in L^{1}(\Omega), f(x, u) \geqq 0$, then formally $v$ is a solution of

$$
-\Delta_{p} v=\lambda f(x, H(v))(1+g(v))^{p-1} .
$$

Conversely, if $v$ is a solution of a problem of the form

$$
-\Delta_{p} v=\lambda f(x, v)(1+g(v))^{p-1}
$$

then formally $u$ is a solution of

$$
-\Delta_{p} u=\beta(u)|\nabla u|^{p}+\lambda f(x, \Psi(u)) .
$$

This extends strongly the domain of applications of our result.

Remark 7.8 This argument was an essential point in the Proof of Theorem 1.3: we used the fact that, for any $g$ satisfying (1.2) with $\Lambda=\infty$, and any $v \in \mathcal{W}(\Omega)$, such that $-\Delta_{p} v=F \geqq 0$, then $u=H(v) \in \mathcal{W}$ and is a solution of equation $-\Delta_{p} u=\beta(u)|\nabla u|^{p}+F e^{-\gamma(u)}$.

Let us give a simple example of application:

Corollary 7.9 Let $\omega \in C^{1}([0, \infty))$ be nonnegative and nondecreasing, and $f \in L^{r}(\Omega), r>N / p$. Consider the problem

$$
-\Delta_{p} u=(p-1)|\nabla u|^{p}+\lambda f(x)(1+\omega(u))^{p-1}, \quad u=0 \quad \text { on } \partial \Omega .
$$

(i) Then for small $\lambda>0$, there exists a solution in $W_{0}^{1, p}(\Omega) \cap L^{\infty}(\Omega)$.

(ii) Assume that $\lim \sup _{t \rightarrow \infty} \omega(t)^{p-1} / e^{k t}<\infty$ for some $k>0$.

If $r^{\prime}(k+1)<N /(N-p)$ then for any small $\lambda>0$, there exists an infinity of solutions in $W_{0}^{1, p}(\Omega)$.

If $r^{\prime}\left(k / p^{\prime}+1\right)<N /(N-p)$ and $\omega$ is convex, there exists two solutions in $W_{0}^{1, p}(\Omega) \cap L^{\infty}(\Omega)$.

Proof. Setting $v=e^{u}-1$, then $v$ satisfies the equation $-\Delta_{p} v=\lambda f(x)(1+\tilde{g}(v))^{p-1}$ in $\Omega$, where $1+\tilde{g}(v)=(1+v)(1+\omega(\ln (1+v)))$. And $\tilde{g}$ satisfies (1.3) with $Q=(p-1)(k+1)$, and is convex when $\omega$ is convex. The results follows from Proposition 5.1, Theorems 1.1, 1.6 and 1.5. 
Remark 7.10 In particular for any $b>0$, for any $f \in L^{r}(\Omega), r>N / p$, and small $\lambda>0$, problem

$$
-\Delta_{p} u=|\nabla u|^{p}+\lambda f(x)(1+u)^{b} \quad \text { in } \Omega, \quad u=0 \quad \text { on } \partial \Omega,
$$

has an infinity of solutions in $W_{0}^{1, p}(\Omega)$, one of them in $L^{\infty}(\Omega)$, two of them if $b \geqq p-1$.

2) Theorem 1.1 also covers and precises the recent multiplicity result of [1, Theorem 3.1], relative to radial solutions of problems with other powers of the gradient:

Corollary 7.11 Let $\Omega=B(0,1)$. Consider the problem

$$
-\Delta_{m} w=c|\nabla w|^{q}+\lambda f \quad \text { in } \Omega, \quad w=0 \quad \text { on } \partial \Omega,
$$

with $m>1$ and $q \geqq(m-1) N /(N-1)$, where $f$ is radial and $f \in L^{r}(\Omega), r>N(q-m+1) / q$, and $c>0$. Then there exists $\tilde{\lambda}>0$ such that for any $\lambda<\tilde{\lambda}$, problem (7.5) in $\mathcal{D}^{\prime}(\Omega)$ admits an infinity of radial solutions, and one of them in $C^{1}(\bar{\Omega})$.

Proof. In the radial case, problem (7.5) only involves the derivative $w^{\prime}$ :

$$
-r^{1-N}\left(r^{N-1}\left|w^{\prime}\right|^{m-2} w^{\prime}\right)^{\prime}=c\left|w^{\prime}\right|^{q}+\lambda f
$$

hence the change of functions $w^{\prime}=A\left|u^{\prime}\right|^{p / q-1} u^{\prime}$ with $p=q /(q-m+1)$ and $A=\left(c /(p-1)^{-p / q}\right.$. reduces formally to

$$
-r^{1-N}\left(r^{N-1}\left|u^{\prime}\right|^{p-2} u^{\prime}\right)^{\prime}=\left((p-1)\left|u^{\prime}\right|^{p}+\rho f,\right.
$$

where $\rho=(c /(p-1))^{p-1} \lambda$. By hypothesis, $1<p \leqq N$, and $f \in L^{r}(\Omega), r>N / p$. From Theorem 1.2. for any $\rho<\lambda_{1}(f)$ defined at (1.16), and for any measure $\mu_{s} \in \mathcal{M}_{s}^{+}(B(0,1))$ there exists a renormalized nonnegative solution $v_{s}$ of problem

$$
-\Delta_{p} v_{s}=\rho f\left(1+v_{s}\right)^{p-1}+\mu_{s} \quad \text { in } \Omega, \quad v_{s}=0 \quad \text { on } \partial \Omega
$$

thus there exists an infinity of nonnegative solutions $u_{s}=\ln \left(1+v_{s}\right) \in W_{0}^{1, p}(\Omega)$ of

$$
-\Delta_{p} u_{s}=(p-1)\left|\nabla u_{s}\right|^{p}+\rho f \quad \text { in } \Omega .
$$

Take $\mu_{s, a}=a \delta_{0}$, with $a>0$. Then (7.8) has at least a radial solution $v_{s, a}$, obtained as in Theorem 6.2 by the Schauder theorem for radial functions. Then $u=u_{s, a}$ is radial, and $r \mapsto u(r)$ satisfies (7.7) in $\mathcal{D}^{\prime}((0,1))$, hence $u \in C^{1}((0,1])$ and $u^{\prime}(r)<0$. Then $w(r)=-A \int_{r}^{1}\left|u^{\prime}\right|^{p / q-1} u^{\prime} d s \in C^{1}((0,1])$, $w(r) \geqq 0$ and $w$ satisfies (7.8) in $\mathcal{D}^{\prime}((0,1))$ with $\lambda=((p-1) / c)^{p-1} \rho$. Moreover $x \mapsto w^{\prime}(|x|) \in$ $L^{q}(\Omega \backslash\{0\})$, and $\{0\}$ has a $p$-capacity 0 since $p \leqq N$, thus $w \in W_{0}^{1, q}(\Omega)$, hence $|\nabla w|^{m-1} \in L^{p^{\prime}}(\Omega)$. Let $\varphi \in \mathcal{D}(\Omega)$ and $\varphi_{n} \in \mathcal{D}\left((\Omega \backslash\{0\})\right.$ converging to $\varphi$ in $W_{0}^{1, p}(\Omega)$. Then

$$
\begin{aligned}
\int_{\Omega}|\nabla w|^{m-2} \nabla w \cdot \nabla \varphi_{n} d x & =A^{m-1} \int_{\Omega}|\nabla u|^{p-2} \nabla u \cdot \nabla \varphi_{n} d x \\
& =A^{m-1} \int_{\Omega}\left((p-1)|\nabla u|^{p}+\rho f\right) \varphi_{n} d x=\int_{\Omega}\left(c|\nabla w|^{q}+\rho f\right) \varphi_{n} d x ;
\end{aligned}
$$


going to the limit, we find that $w$ is a solution of $(\overline{7.5})$ in $\mathcal{D}^{\prime}(\Omega)$. Then there exists an infinity of radial solutions of (7.5) for any $\lambda<\tilde{\lambda}=((p-1) / c)^{p-1} \lambda_{1}(f)$. And taking $\mu_{s, a}=0$, the problem in $u$ admits a bounded radial solution $u_{0} \in C^{1}([0,1])$, thus (7.5) admits a radial solution $w_{0} \in C^{1}(\bar{\Omega})$.

Remark 7.12 Moreover, since $v_{s}$ is radial, from the assumptions on $f$, we know the precise behaviour near 0 of the singular solutions:

If $q>(m-1) N /(N-1)$, in otherwords $p<N$, then $v(r)=c_{N, p} a r^{(p-N) /(p-1)}(1+o(1))$ near 0 , with $c_{N, p}=(p-1)(n-p)^{-1}\left|S_{N-1}\right|^{-1 /(p-1)} ;$ and $v^{\prime}(r)=c_{N} a(p-N)(p-1)^{-1} r^{(1-N)(p-1)}(1+o(1))$. And $u^{\prime}=v^{\prime} /(1+v)$, thus $\left|u^{\prime}\right|^{p / q-1} u^{\prime}=-((N-p) /(p-1) r)^{-p / q}(1+o(1))$. If $q>m$, that means if $q>p$, then $w$ is bounded, the singularity appears at the level of the gradient. If $q<m$, then $w(r)=$ $C r^{-(m-q) /(q-m+1)}(1+o(1))$, with $C=C(N, m, q, c)$. If $q=m-1$, then $w(r)=C(-\ln r)^{-1}(1+o(1))$.

If $q=(m-1) N /(N-1)$, then $p=N$, and $\lim _{r \rightarrow 0}(-\ln r)^{-1} v(r)=c_{N} a$ with $c_{N}=\left|S_{N-1}\right|^{-1 /(N-1)}$, $\lim _{r \rightarrow 0} r v^{\prime}(r)=-c_{N} a,\left|u^{\prime}\right|^{p / q-1} u^{\prime}=-(r(-\ln r))^{-(N-1) /(m-1)}(1+o(1))$. If $N<m$, then $w$ is bounded; if $N>m$, then $w=C(-\ln r)^{-(N-1) /(m-1)} r^{-(N-m) /(m-1)}(1+o(1))$, with $C=C(N, m, c)$. if $N=m$, then $w=C(\ln (-\ln r)(1+o(1))$.

\section{Appendix}

Proof of Lemma 2.12. The relation is known for $V \in W_{0}^{1, p}(\Omega)$, see for example [3]. Let $F=-\Delta_{p} V$, and $F_{n}=\min (F, n) \in L^{\infty}(\Omega)$, and $V_{n}=\mathcal{G}\left(F_{n}\right)$. Then $F_{n} \rightarrow F$ in $L^{1}(\Omega)$. And $\left(V_{n}\right)$ is nondecreasing; from Remark 2.11, $\left(V_{n}\right)$ converges a.e. to a renormalized solution $w$ of $-\Delta_{p} v=-\Delta_{p} V$; from uniqueness, $w=V$; and

$$
\int_{\Omega}|\nabla U|^{p} d x \geqq \int_{\Omega} U^{p} V_{n}^{1-p}\left(-\Delta_{p} V_{n}\right) d x .
$$

From the Fatou Lemma $U^{p} V^{1-p}\left(-\Delta_{p} V\right) \in L^{1}(\Omega)$, and (2.11) holds.

Proof of Lemma 2.13. We have $\bar{m} \in(1, N / p)$ for $p<N$, and $\bar{m}=1$ for $p=N$.

- First suppose $1<m<N / p$, thus $p<N$. Let $\varepsilon>0$ and $k>0$. We use the test function $\phi_{\beta, \varepsilon}\left(T_{k}(U)\right)$, where $\phi_{\beta, \varepsilon}(w)=\int_{0}^{w}(\varepsilon+|t|)^{-\beta} d t$, for given real $\beta<1$. We get

$$
\int_{\Omega} \frac{\left|\nabla T_{k}(U)\right|^{p}}{\left(\varepsilon+\left|T_{k}(U)\right|\right)^{\beta}} d x \leqq(1-\beta)^{-1} \int_{\Omega}|F|\left(\varepsilon+\left|T_{k}(U)\right|\right)^{1-\beta} d x
$$

Setting $\eta=(p-1) m N /(N-m)$ and then $\eta^{*}=(p-1) N m /(N-p m)$, we take

$$
\beta=1-\frac{\eta^{*}}{m^{\prime}}=\frac{N p(\bar{m}-m)}{\bar{m}(N-p m)}, \quad \alpha=1-\frac{\beta}{p}=\frac{\eta^{*}}{p^{*}}
$$


then $\beta, \alpha \in(0,1)$ for $m<\bar{m}$, and $\beta \leqq 0 \leqq \alpha-1$ for $m \geqq \bar{m}$. The function $U_{k, \varepsilon}=\left(\left(\varepsilon+\left|T_{k}(U)\right|\right)^{\alpha}-\right.$ $\left.\varepsilon^{\alpha}\right) \operatorname{sign}(U)$ belongs to $W_{0}^{1, p}(\Omega)$, and from (8.1) we get

$$
\begin{aligned}
\int_{\Omega}\left|\nabla U_{k, \varepsilon}\right|^{p} d x & =\alpha^{p} \int_{\Omega} \frac{\left|\nabla T_{k}(U)\right|^{p}}{\left(\varepsilon+\left|T_{k}(U)\right|\right)^{\beta}} d x \leqq C \int_{\Omega}|F|\left(\varepsilon^{\alpha}+\left|U_{k, \varepsilon}\right|\right)^{\eta^{*} / \alpha m^{\prime}} d x \\
& \leqq C\|F\|_{L^{m}(\Omega)}\left(\int_{\Omega}\left(\varepsilon^{\alpha}+\left|U_{k, \varepsilon}\right|\right)^{\eta^{*} / \alpha} d x\right)^{\frac{1}{m^{\prime}}}
\end{aligned}
$$

where $C>0$. From the Sobolev injection of $W_{0}^{1, p}(\Omega)$ into $L^{p^{*}}(\Omega)$ we find, with other constants $C>0$, depending on $\Omega$

$$
\begin{aligned}
\left(\int_{\Omega}\left(\varepsilon^{\alpha}+\left|U_{k, \varepsilon}\right|\right)^{p^{*}} d x\right)^{p / p *} & \leqq C\left(\varepsilon^{\alpha p}+\left(\int_{\Omega}\left|U_{k, \varepsilon}\right|^{p^{*}} d x\right)^{p / p *}\right. \\
& \leqq C\left(\varepsilon^{\alpha p}+\|F\|_{L^{m}(\Omega)}\left(\int_{\Omega}\left(\varepsilon^{\alpha}+\left|U_{k, \varepsilon}\right|\right)^{p^{*}} d x\right)^{\frac{1}{m^{\prime}}},\right.
\end{aligned}
$$

and $p^{*}<p m^{\prime}$, because $m<N / p$; thus from the Young inequality

$$
\int_{\Omega}\left|T_{k}(U)\right|^{\eta^{*}} d x \leqq \int_{\Omega}\left(\varepsilon^{\alpha}+\left|U_{k, \varepsilon}\right|\right)^{p^{*}} d x \leqq C\left(\varepsilon^{\eta^{*}}+\|F\|_{L^{m}(\Omega)}^{1 /\left(p / p^{*}-1 / m^{\prime}\right)}\right) .
$$

And $1 /\left(p / p^{*}-1 / m^{\prime}\right)=\eta^{*} /(p-1)$, thus

$$
\int_{\Omega}\left|T_{k}(U)\right|^{\eta^{*}} d x \leqq C\|F\|_{L^{m}(\Omega)}^{\eta^{*} /(p-1)}
$$

and from the Fatou Lemma, (iii) follows:

$$
\left(\int_{\Omega}|U|^{(p-1) N m /(N-p m)} d x\right)^{(N-p m) / N m} \leqq C\|F\|_{L^{m}(\Omega)} .
$$

- Assume moreover that $m<\bar{m}$. Using (8.2), (8.3) and going to the limit as $k \longrightarrow \infty$, we find

$$
\int_{\Omega} \frac{|\nabla U|^{p}}{(\varepsilon+\mid U) \mid)^{\beta}} d x \leqq C\left(\|F\|_{L^{m}(\Omega)} \varepsilon^{\eta^{*} / m^{\prime}}+\|F\|_{L^{m}(\Omega)}^{\eta^{*} p / p^{*}(p-1)}\right) .
$$

We have $\eta<p$, and $\beta \eta /(p-\eta)=\eta^{*}$, thus from Hölder inequality,

$$
\left.\int_{\Omega}|\nabla U|^{\eta} d x \leqq\left(\int_{\Omega} \frac{|\nabla U|^{p}}{(\varepsilon+\mid U) \mid)^{\beta}} d x\right)^{\eta / p}\left(\int_{\Omega}(\varepsilon+\mid U) \mid\right)^{\eta^{*}} d x\right)^{1-\eta / p} .
$$

Using (8.5) and (8.4), and going at the limit as $\varepsilon \longrightarrow 0$, (iv) follows for $m<\bar{m}$ :

$$
\left(\int_{\Omega}|\nabla U|^{(p-1) N m /(N-m)} d x\right)^{(N-m) / N m} \leqq C\|F\|_{L^{m}(\Omega)}
$$


- Assume $m \geqq \bar{m}, p<N$. Then $L^{m}(\Omega) \subset W^{-1, p^{\prime}}(\Omega)$, thus, from uniqueness, $U \in W_{0}^{1, p}(\Omega)$ and it is a variational solution. More precisely, $L^{m}(\Omega) \subset W^{-1, N m /(N-m)}(\Omega)$. If $m=\bar{m}$, then $N m /(N-m)=$ $p^{\prime}$, and (8.6) follows. If $m>\bar{m}$, then from [46], [47], $U \in W^{1, \ell}(\Omega)$ with $\ell=(p-1) N m /(N-m)$, and (8.6) still holds, and (iii) follows for $m \geqq \bar{m}$; and (i) and (ii) from the Sobolev injection. Another proof in case $m>N / p$ is given in [56].

- Assume $m>1$ and $p=N$. then again $L^{m}(\Omega) \subset W^{-1, N m /(N-m)}(\Omega)$, hence (8.6) still holds, and then $U \in L^{\infty}(\Omega)$.

Proof of Proposition 2.14. If $p=N$, then $U \in L^{\sigma}(\Omega)$ for any $\sigma \geqq 1$ from Remark 2.3. then $f(x)|U|^{Q} \in L^{m}(\Omega)$ for any $m \in(1, r)$, then $U \in W_{0}^{1, N}(\Omega) \cap L^{\infty}(\Omega)$ from Lemma 2.13, and $|\nabla U|^{N-1} \in L^{\tau}(\Omega)$ for $\tau=N m /(N-m)$.

Next suppose $p<N$. First assume that $Q r^{\prime}<Q_{1}$. Let $k \in(0,1)$ such that $1 / r^{\prime}>k+$ $Q(N-p) / N(p-1)$. Then $f(x)|U|^{Q} \in L^{m_{0}}(\Omega)$ with $m_{0}=1 /(1-k)>1$. Taking $k$ small enough, one finds $m_{0}<N / p$, thus $h(x) \in L^{m_{0}}(\Omega)$, then from Lemma 2.13, $|U|^{s_{1}} \in L^{1}(\Omega)$ with $s_{1}=$ $(p-1) N m_{0} /\left(N-p m_{0}\right)$. Then $f(x)|U|^{Q} \in L^{m_{1}}(\Omega)$, where

$$
\frac{1}{m_{1}}-\frac{1}{r}=\frac{Q}{p-1}\left(\frac{1}{m_{0}}-\frac{p}{N}\right) .
$$

And $1 / m_{1}-1 / m_{0}<(Q-p+1)\left(1-m_{0}\right) / m_{0}(p-1)<0$, hence $m_{1}>m_{0}$. For any $n \in \mathbb{N}$ such that $f(x)|U|^{q} \in L^{m_{n}}(\Omega)$ and $m_{n}<N / p$, we can define $m_{n+1}$ by

$$
\frac{1}{m_{n+1}}-\frac{1}{r}=\frac{Q}{p-1}\left(\frac{1}{m_{n}}-\frac{p}{N}\right) .
$$

and $m_{n}<m_{n+1}$. If $m_{n}<N / p$ for any $n$, it has a limit $m$, then $m=(Q /(p-1)-1) /\left(Q p^{\prime} / N-1 / r\right)$. When $Q \geqq p-1$, then $m<1$, which is impossible. Then after a finite number $\bar{n}$ of steps we arrive to $m_{\bar{n}}>N / p$, thus (i) follows from Lemma 2.13,

Next assume $Q>p-1$ and $Q r^{\prime}=Q_{1}$, thus $p<N$, and $|U|^{p-1} \in L^{\sigma}(\Omega)$ for some $\sigma>N /(N-p)$. Setting $\sigma=(1+\theta) N /(N-p)$ with $\theta>0$, and $m_{0}=(1+\theta r) /(1+\theta)>1$, there holds $Q r /\left(r-m_{0}\right)=\sigma$, and from Hölder inequality:

$$
\int_{\Omega}\left(f|U|^{q}\right)^{m_{0}} d x \leqq\left(\int_{\Omega} f^{r} d x\right)^{m_{0} / r}\left(\int_{\Omega}|U|^{q r /\left(r-m_{0}\right)} d x\right)^{1-m_{0} / r}
$$

thus we still have $f(x)|U|^{Q} \in L^{m_{0}}(\Omega)$ and $1 / m_{1}-1 / m_{0}=(Q-p+1)\left(1-m_{0}\right) / m_{0}(p-1)<0$, thus (ii) follows as above.

And (iii) follows from [40, Propositions 1.2 and 1.3]. Indeed the equation can be written under the form

$$
-\Delta_{p} U=K(x)\left(1+|U|^{p-1}\right),
$$


where $|K(x)| \leqq f(x)\left(1+|U|^{Q-p+1}\right)$; if $(Q+1) r^{\prime} \leqq p^{*}$ then $K(x) \in L^{s}(\Omega)$ for some $s \geqq N / p$, then $U \in L^{\infty}(\Omega)$ if the inequality is strict, and $U \in L^{k}(\Omega)$ for any $k \geqq 1$ in case of equality.

Next assume $Q<p-1$. Then $|h(x)| \leqq f(x)\left(|U|^{p-1}+2\right)$, hence (iv) holds from above. If $r=N / p$, then again $Q r^{\prime}<Q_{1}$, and we find $m=N / p$. Then $h(x) \in L^{s}(\Omega)$ for any $s<N / p$, hence $U \in W_{0}^{1, p}(\Omega)$ and (v) holds from Lemma 2.13. If $r<N / p$ then $m<N / p$. Thus from Lemma 2.13 $U^{k} \in L^{1}(\Omega)$ for any $k<(p-1) N m /(N-p m)=\theta$. If $(Q+1) r^{\prime}<Q_{1}$, then $m>\bar{m}$, thus $U \in W_{0}^{1, p}(\Omega)$. If $(Q+1) r^{\prime} \geqq Q_{1}$, then $m \leqq \bar{m}$, thus $|\nabla U|^{p-1} \in L^{\tau}(\Omega)$ for any $\tau<N m /(N-m)=\theta$. Then (vi) follows.

Proof of Lemma 2.16. In [10, Proposition 2.1], we have given the estimates (2.13) for the superharmonic continuous functions in $\mathbb{R}^{N}$. In fact they adapt to any local renormalized solution of the equation in $\Omega$. Indeed such a solution satisfies $U^{p-1} \in L_{l o c}^{\sigma}(\Omega)$ for any $\sigma \in(0, N /(N-p))$. Let $x_{0} \in \Omega$ and $\rho>0$ such that $B\left(x_{0}, 4 \rho\right) \subset \Omega$. Let $\varphi_{\rho}=\xi_{\rho}^{\lambda}$ with $\lambda>0$ large enough, and $\xi_{\rho}(x)=\zeta\left(\left|x-x_{0}\right| / \rho\right)$, where $\zeta_{\rho} \in \mathcal{D}(\mathbb{R})$ with values in $[0,1]$, such that $\xi(t)=1$ for $|t| \leq 1,0$ for $|t| \geq 2$. Let $\sigma \in(1, N /(N-p))$ and $\alpha \in(1-p, 0)$. We set $U_{\varepsilon}=U+\varepsilon$, for any $\varepsilon>0$. Let $k>\varepsilon$. Then we can take

$$
\phi=T_{k}\left(U_{\varepsilon}\right)^{\alpha} \xi_{\rho}^{\lambda}
$$

as a test function, where $\lambda>0$ large enough will be fixed after. Hence

$$
\begin{gathered}
\int_{\Omega} F T_{k}\left(U_{\varepsilon}\right)^{\alpha} \xi_{\rho}^{\lambda} d x+|\alpha| \int_{\Omega} T_{k}\left(U_{\varepsilon}\right)^{\alpha-1} \xi_{\rho}^{\lambda} \mid \nabla\left(\left.T_{k}(U)\right|^{p} d x\right. \\
\leq \lambda \int_{\Omega} T_{k}\left(U_{\varepsilon}\right)^{\alpha} \xi_{\rho}^{\lambda-1} \mid \nabla\left(T _ { k } ( U ) | ^ { p - 2 } \nabla \left(T_{k}(U) \nabla \xi_{\rho} d x\right.\right. \\
\leq \frac{|\alpha|}{2} \int_{\Omega} T_{k}\left(U_{\varepsilon}\right)^{\alpha-1} \xi_{\rho}^{\lambda} \mid \nabla\left(\left.T_{k}(U)\right|^{p} d x+C(\alpha) \int_{\Omega} T_{k}\left(U_{\varepsilon}\right)^{\alpha+p-1} \xi_{\rho}^{\lambda-p}\left|\nabla \xi_{\rho}\right|^{p} d x .\right.
\end{gathered}
$$

Hence

$$
\int_{\Omega} F T_{k}\left(U_{\varepsilon}\right)^{\alpha} \xi_{\rho}^{\lambda} d x+\frac{|\alpha|}{2} \int_{\Omega} T_{k}\left(U_{\varepsilon}\right)^{\alpha-1} \xi_{\rho}^{\lambda} \mid \nabla\left(\left.T_{k}(U)\right|^{p} d x \leq C(\alpha) \int_{\Omega} T_{k}\left(U_{\varepsilon}\right)^{\alpha+p-1} \xi_{\rho}^{\lambda-p}\left|\nabla \xi_{\rho}\right|^{p} d x .\right.
$$

Then we make $\varepsilon$ tend to 0 and $k$ to $\infty$. Setting $\theta=(p-1) \sigma /(p-1+\alpha)>1$, we obtain

$$
\int_{\Omega} F U^{\alpha} \xi_{\rho}^{\lambda} d x+\frac{|\alpha|}{2} \int_{\Omega} U^{\alpha-1} \xi_{\rho}^{\lambda}|\nabla U|^{p} d x \leq C\left(\int_{\operatorname{supp} \nabla \zeta} U^{(p-1) \sigma} \xi_{\rho}^{\lambda} d x\right)^{1 / \theta}\left(\int_{\Omega} \xi_{\rho}^{\lambda-p \theta^{\prime}}\left|\nabla \xi_{\rho}\right|^{p \theta^{\prime}} d x\right)^{1 / \theta^{\prime}}
$$

with a new constant $C$ depending of $\alpha$ from the Hölder inequality. Taking $\lambda$ large enough,

$$
\int_{\Omega} F U^{\alpha} \xi_{\rho}^{\lambda}+\frac{|\alpha|}{2} \int_{\Omega} U^{\alpha-1} \xi_{\rho}^{\lambda}|\nabla U|^{p} \leq C \rho^{N / \theta^{\prime}-p}\left(\int_{\operatorname{supp} \nabla \xi_{\rho}} U^{(p-1) \sigma} \xi_{\rho}^{\lambda} d x\right)^{1 / \theta}
$$


Next we take $\phi=\xi_{\rho}^{\lambda}$ as a test function. We get

$$
\begin{aligned}
\int_{\Omega} F \xi_{\rho}^{\lambda} d x & \leq \lambda \int_{\Omega} \xi_{\rho}^{\lambda-1}|\nabla U|^{p-2} \nabla U . \nabla \xi_{\rho} d x \\
& \leqq \lambda\left(\int_{\Omega} U_{\varepsilon}^{\alpha-1} \xi_{\rho}^{\lambda}|\nabla U|^{p} d x\right)^{1 / p^{\prime}}\left(\int_{\Omega} U_{\varepsilon}^{(1-\alpha)(p-1)} \xi_{\rho}^{\lambda-p}\left|\nabla \xi_{\rho}\right|^{p} d x\right)^{1 / p} .
\end{aligned}
$$

Since $\ell>p-1$, we can fix an $\alpha \in(1-p, 0)$ such that $\tau=\sigma /(1-\alpha)>1$. Then as $\varepsilon \rightarrow 0$,

$$
\begin{aligned}
\int_{\Omega} F \xi_{\rho}^{\lambda} d x & \leq C\left(\int_{\operatorname{supp} \nabla \xi_{\rho}} U^{(p-1) \sigma} \xi_{\rho}^{\lambda} d x\right)^{1 / \theta p^{\prime}+1 / \tau p} \\
& \times\left(\int_{\Omega} \xi_{\rho}^{\lambda-\theta^{\prime} p}\left|\nabla \xi_{\rho}\right|^{\theta^{\prime} p} d x\right)^{1 / \theta^{\prime} p^{\prime}}\left(\int_{\Omega} \xi_{\rho}^{\lambda-\tau^{\prime} p}\left|\nabla \xi_{\rho}\right|^{\tau^{\prime} p} d x\right)^{1 / \tau^{\prime} p} .
\end{aligned}
$$

But $1 / \theta p^{\prime}+1 / \tau p=\sigma=1-\left(1 / \theta^{\prime} p^{\prime}+1 / \tau^{\prime} p\right)$, hence

$$
\int_{\Omega} F \xi_{\rho}^{\lambda} d x \leq C\left(\int_{\operatorname{supp} \nabla \xi_{\rho}} U^{(p-1) \sigma} \xi_{\rho}^{\lambda} d x\right)^{1 / \sigma} \rho^{N(1-1 / \sigma)-p}
$$

and (2.13) follows. Otherwise, if $U \in W_{l o c}^{1, p}(\Omega)$, from the weak Harnack inequality, there exists a constant $C^{\prime}=C^{\prime}(\sigma, N, p)$ such that

$$
\left(\frac{1}{\rho^{N}} \int_{B\left(x_{0}, 2 \rho\right)} U^{(p-1) \sigma} d x\right)^{1 /(p-1) \sigma} \leqq C^{\prime} \inf \operatorname{ess}_{B\left(x_{0}, \rho\right)} U
$$

hence (2.14) holds by fixing $\sigma$.

Proof of Lemma 2.17. Let $\hat{h}(x, t)=h(x, \max (0, \min (t, u(x)))$. Then $0 \leqq \hat{h}(x, t) \leqq F(x)$ a.e. in $\Omega$. From the Schauder theorem for any $n \in \mathbb{N}$ there exists $V_{n} \in W_{0}^{1, p}(\Omega) \cap L^{\infty}(\Omega)$ such that

$$
-\Delta_{p} V_{n}=T_{n}\left(\hat{h}\left(x, V_{n}\right)\right) \quad \text { in } \Omega .
$$

From Remark 2.11, up to a subsequence, $V_{n}$ converges a.e. to a renormalized solution $V$ of equation

$$
-\Delta_{p} V=\hat{h}(x, V) \quad \text { in } \Omega,
$$

and $V \geqq 0$ from the Maximum Principle. It remains to show that $V \leqq U$. For fixed $m>0$, and $n \in \mathbb{N}$ the function $\omega=T_{m}\left(\left(V_{n}-U\right)^{+}\right)=T_{m}\left(\left(V_{n}-T_{\left\|V_{n}\right\|_{L^{\infty}(\Omega)}} U\right)^{+}\right) \in W_{0}^{1, p}(\Omega)$; and $\omega^{+}=\omega^{-}=0$, thus from [25, Definition 2.13]

$$
\int_{\Omega}|\nabla U|^{p-2} \nabla U \cdot \nabla \omega d x=\int_{\Omega} \omega h(x, U) d x+\int_{\Omega} \omega^{+} d \mu_{s}^{+}-\int_{\Omega} \omega^{-} d \mu_{s}^{-}=\int_{\Omega} \omega h(x, U) d x
$$


Otherwise, $\omega$ is also admissible in the equation relative to $V_{n}$ :

$$
\int_{\Omega}\left|\nabla V_{n}\right|^{p-2} \nabla V_{n} \cdot \nabla \omega d x=\int_{\Omega} T_{n}\left(\hat{h}\left(x, V_{n}\right)\right) \omega d x
$$

then

$$
\begin{aligned}
& \int_{\Omega}\left(\left|\nabla V_{n}\right|^{p-2} \nabla V_{n}-|\nabla U|^{p-2} \nabla U\right) \cdot \nabla\left(T_{m}\left(\left(V_{n}-U\right)^{+}\right)\right) d x \\
& =\int_{\Omega}\left(T_{n}\left(\hat{h}\left(x, V_{n}\right)-h(x, U)\right) T_{m}\left(\left(V_{n}-U\right)^{+}\right) d x \leqq \int_{\Omega}\left(h(x, U)-T_{n}(h(x, U)) T_{m}\left(\left(V_{n}-U\right)^{+}\right) d x .\right.\right.
\end{aligned}
$$

From the Fatou Lemma and Lebesgue Theorem, going to the limit as $n \longrightarrow \infty$ for fixed $m$, since the truncations converge strongly in $W_{0}^{1, p}(\Omega)$, we deduce

$$
\int_{\Omega}\left(|\nabla V|^{p-2} \nabla V-|\nabla U|^{p-2} \nabla U\right) \cdot \nabla\left(T_{m}\left((V-U)^{+}\right)\right) d x \leqq 0 .
$$

Then $T_{m}\left((V-U)^{+}=0\right.$ for any $m>0$, thus $V \leqq U$ a.e. in $\Omega$.

\section{References}

[1] Abdellaoui B., Multiplicity result for quasilinear elliptic problems with general growth of the gradient, Advances Nonlinear Studies, 8 (2008), 289-301.

[2] Abdellaoui B., Dall'Aglio A., and Peral I., Some remarks on elliptic problems with critical growth in the gradient, J. Diff. Equ.,222 (2006), 21-62.

[3] Allegretto W., and Huang Y.X., A Picone's identity for the p-Laplacian and Applications, Nonlinear Anal. 32 (1998), 819-830.

[4] Alvino A., Boccardo L., Ferone V., Orsina L., and Trombetti G., Existence results for nonlinear elliptic equations with degenerate coercivity, Ann. Mat. Pura Appl. (4), 182 (2003), 53-79.

[5] Alvino A., Ferone V., and Trombetti G., Estimates for the gradient of solutions of nonlinear elliptic equations with $L^{1}$ data, Ann. Mat. Pura Appl. 178 (2000), 129-142.

[6] Ambrosetti A., Brezis H, and Cerami G., Combined effects of concave and convex nonlinearity in some elliptic problems, J. Funct. Anal. 122 (1994) 519-543.

[7] Benilan P., Boccardo L., Gallouet T., Gariepy R., Pierre M., and Vazquez J., An L $L^{1}$ theory of existence uniqueness of nonlinear elliptic equations, Ann. Scuola Norm. Sup. Pisa, 28 (1995), 241-273 
[8] Benilan P., Brézis H., and Crandall M., A semilinear elliptic equation in $L^{1}\left(\mathbb{R}^{N}\right)$, Ann. Scuola Norm. Sup. Pisa, 2 (1975), 523-555.

[9] Bidaut-Véron M.F., Removable singularities and existence for a quasilinear equation, Adv. Nonlinear Studies 3 (2003), 25-63

[10] Bidaut-Véron M.F., and Pohozaev S., Nonexistence results and estimates for some nonlinear elliptic problems, J. Anal. Mathématique, 84 (2001), 1-49.

[11] Boccardo L., and Gallouet T., Nonlinear elliptic and parabolic equations involving measure data, J Funct. Anal. 87 (1989), 149-169.

[12] Boccardo L, Gallouet T., and Orsina L., Existence and uniqueness of entropy solutions for nonlinear elliptic equations with measure data, Ann. Inst. H. Poincaré Anal. non Lin. 13 (1996), 539-555.

[13] Boccardo L., Murat F., Puel J., Résultats d'existence pour certains problèmes elliptiques quasilinéaires, Ann. Scuola. Norm. Sup. Pisa 11 (2) (1984) 213-235.

[14] Boccardo L., Segura de León S., Trombetti C., Bounded and unbounded solutions for a class of quasi-linear elliptic problems with a quadratic gradient term, J. Math. Pures Appl. 80 (9) (2001) 919-940.

[15] Brezis H., Cazenave T., Martel Y., and Ramiandrisoa A., Blow-up for $u_{t}-\Delta u=g(u)$ revisited, Adv. Diff. Eq. 1 (1996), 73-90

[16] Brezis H., and Vazquez J., Blow-up solutions of some nonlinear elliptic problems, Rev. Math. Univ. Complutense Madrid 10 (1997), 443-469.

[17] Cabre X., Extremal solutions and instantaneous complete blow-up for elliptic and parabolic problems, Contemporary Mathematics, American Math. Soc. 2007, in: Perspectives in Nonlinear Partial Differential Equations, in honor of H. Brezis.

[18] Cabre X., Capella A., and Sanchon M., Regularity of radial minimizers of rection equations involving the p-Laplacian, preprint arXiv:0712.2788.

[19] Cabre X., and Sanchon M., Semi-stable and extremal solutions of reaction equations involving the p-Laplacian, Comm. Pure Appl. Anal. 6 (2007), 43-67.

[20] Crandall M., and Rabinowitz P., Some continuation and variational methods for positive solutions of nonlinear elliptic eigenvalue problems, Arch. Rat. Mech. Anal. 58 (1975), 207-218

[21] Cuesta M., Eigenvalue problems for the p-Laplacian with indefinite weights, Electronic J. Diff. Equ. 33 (2001), 1-9. 
[22] Dall'Aglio A. Approximate solutions with $L^{1}$ data. Application to the H-convergence of parabolic quasi-linear equations, Ann. Math. Pura Appl. 170 (1996) 207-240.

[23] Dall'Aglio A., Giachetti G., and Segura de Leon S., Nonlinear parabolic problems with a very gen,eral quadratic gradient term, Diff. Int. Equ. 20 (2007), 361-396.

[24] Dal Maso G., and Malusa A., Some properties of reachable solutions of nonlinear elliptic equations with measure data, Ann. Scuola Norm. sup. Pisa, 25 (1997), 375-396.

[25] Dal Maso G., Murat F., Orsina L., and Prignet A., Renormalized solutions of elliptic equations with general measure data, Ann. Scuola Norm. Sup. Pisa, 28 (1999), 741-808.

[26] Droniou J., Porretta A., and Prignet A., Parabolic capacities and soft measures for nonlinear equations, Potential Anal. 19 (2003), 99-161.

[27] Eidelman S., and Eidelman Y., On regularity of the extremal solution of the Dirichlet problem for some semilinear elliptic equations of the second order, Houston J. Math. 31 (2005), 957-960.

[28] Ferrero A., On the solutions of quasilinear elliptic equations with a polynomial-type reaction term, Adv. Diff. Equ. 9 (2004), 1201-1234.

[29] Ferone V., and Murat F., Quasilinear problems having aquadratic growth in the gradient: an existence result when the source term is small, in: Equations aux dérivées partielles et applications, Gauthier-Villars, Paris (1998), 497-515

[30] Ferone V., and Murat F., Nonlinear problems having natural growth in the gradient: an existence result when the source terms are small, Nonlinear Anal. 42 (2000), 1309-1326.

[31] Galaktionov,V.A., On blow-up and degeneracy for the semilinear heat equation with source, Proc. Royal Soc. Edinburgh, 115A (1990), 19-24.

[32] Garcia Azorero J., and Peral I., On an Emden-Fowler type equation, Nonlinear Anal. 18, 11(1992), 1085-1097.

[33] Garcia Azorero J., and Peral I., Some results about the existence of a second positive solution in a quasilinear critical problem, Indiana Univ. Math. J. 43 (1994), 941-957.

[34] Garcia Azorero J., Peral I. and Manfredi J., Sobolev versus Hölder local minimizers and global multiplicity for some quasilinear elliptic equations, Comm. Cont. Math. 3 (2000), 385-404.

[35] Garcia Azorero J., Peral I. and Puel J., Quasilinear problems with exponential growth in the reaction term, Nonlinear Anal. 22 (1994), 481-498.

[36] Dolzmann G., Hungerbühler, N., Müller, S.Uniqueness and maximal regularity for nonlinear elliptic systems of $n$-Laplace type with measure valued right hand side. J. Reine Angew. Math. 520 (2000), 1-35. 
[37] Ghoussoub N., and Preiss, D., A general mountain path principle for locating and classifying critical points, Ann. I.H.P. C, 6,5 (1989), 321-330

[38] Grenon N., L ${ }^{r}$ estimates for degenerate elliptic problems, Potential Anal. 16 (2002), 387-392.

[39] Grenon N., Existence results for semilinear elliptic equations with small measure data, Ann. Inst. H. Poincaré Anal. non Lin. 19 (2002), 1-11.

[40] Guedda M. and Véron L., Quasilinear elliptic equations involving critical Sobolev exponents, Nonlinear Anal. 13 (1989), 879-902.

[41] Horiuchi T., and Kumlin P., On the minimal solution for quasilinear degenerate elliptic equation and its blow-up, J. Math. Kyoto Univ. 44 (2004), 381-439 and 46 (2006), 231-234.

[42] Jeanjean L., On the existence of bounded Palais-Smale sequences and application to a Landesman-Lazer type problem, Proc. Roy. Soc. Edinburg, 4 (1999), 787-809.

[43] Jeanjean L., and Toland, J.F., Bounded Palais-Smale Mountain-path sequences, C.R.Acad.Sci. Paris, 327 (1998), 23-28.

[44] Kilpeläinen T., and Li G., Estimates for p-Poisson equations, Diff. Int. Equ. 13 (2000), 791800.

[45] Kilpeläinen T., Nageswari Shanmugalingam N., and Zhong X., Maximal regularity via reverse Hölder inequalities for elliptic systems of $n$-Laplace type involving measures, Ark. Mat., 46 (2008), 77-93.

[46] Kinnunen J. and Zhou S., A local estimate for nonlinear equations with discontinuous coefficients, Comm. Part. Diff. Equ., 24 (1999), 2043-2068.

[47] Kinnunen J. and Zhou S., A boundary estimate for nonlinear equations with discontinuous coefficients, Diff. Int. Equ. 14 (2001), 475-492.

[48] Lucia M. and Prashant S., Simplicity of principal eigenvalue for p-Laplace operator with singular indefinite weight, Archiv der Math. 86 (2006), 79-89.

[49] Maeda F., Renormalized solutions o Dirichlet problems for quasilinear elliptic equations with general measure data, Hiroshima Math. J., 38 (29008), 51-93.

[50] Malusa A., A new proof of the stability of renormalized solutions to elliptic equations with measure data, Asympt. Anal. 43 (2005), 11-129.

[51] Martel Y., Uniqueness of weak extremal solutions of nonlinear elliptic problems, Houston J. Math 23 (1998), 161-168. 
[52] Mignot F. and Puel J.P., Sur une classe de problèmes non linéaires avec nonlinéarité positive, croissante, convexe, Comm. Part.Diff. Eq. 5 (1980), 791-836

[53] F. Murat, A. Porretta, Stability properties, existence, and nonexistence of renormalized solutionsfor elliptic equations with measure data, Comm. Partial Differential Equations 27 (2002) $2267-2310$.

[54] Nedev G., Regularity of the extremal solution of semilinear elliptic equations, C.R. Acad. Sci. Paris, 330 (2000), 997-2002.

[55] Nedev G., Extremal solutions of semilinear elliptic equations, preprint (2001)

[56] Peral I, Multiplicity of solutions for the p-Laplacian, International Center for Theoretical Physics, Trieste 1997.

[57] Petita F., Ponce A. and Porretta A., New properties of p-Laplacian measure, preprint.

[58] Ponce, A. Personal communication.

[59] Porretta A., Nonlinear equations with natural growth terms and measure data, E.J.D.E., Conference 9 (2002), 183-202.

[60] Porretta A. and Segura de León S., Nonlinear elliptic equations having a gradient term with natural growth, J. Math. Pures Appl. 85 (2006), 465-492.

[61] Raoux T., Thèse de Doctorat, Université de Tours, (1995).

[62] Ramiandrisoa A., Blow-up for two nonlinear problems, Nonlinear Anal. 41 (2000), 8325-854.

[63] Sanchon M., Boundeness of the extremal solutions of some p-Laplacian problems, Nonlinear Anal. 67 (2007), 281-294

[64] Sanchon M., Regularity of the extremal solutions of some Nonlinear elliptic problems, Potential Anal. 27 (2007), 217-224

[65] Segura de León S., Existence and uniqueness for $L^{1}$ data of some elliptic equations with natural growth, Adv. Differential Equations 9 (2003) 1377-1408.

[66] Trudinger N., and Wang X., Quasilinear elliptic equations with signed measure data, Discrete Continuous Dyn. Systems, 23 (2009), 477-494.

[67] Ye D. and Zhou F. Boundedness of the extremal solution for semilinear elliptic problems, Comm. Contemp. Maths. 4 (2002), 547-558 